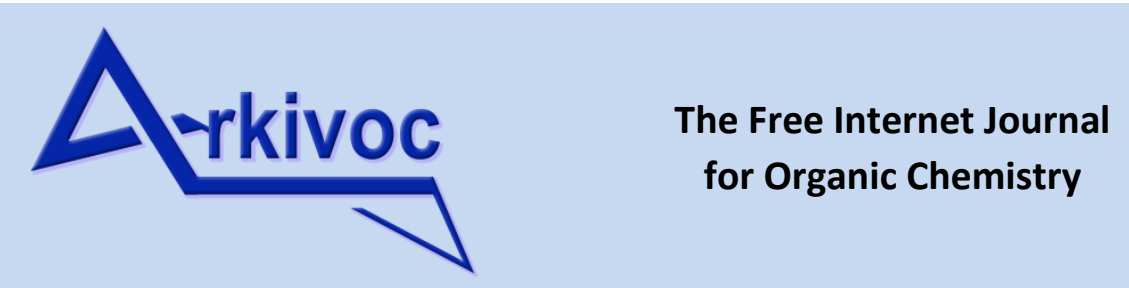

The Free Internet Journal

Archive for

Organic Chemistry

Arkivoc 2020, part vii, 117-144

\title{
Comparative reactivity of 5,7-dimethoxyindoles with aldehydes and ketones
}

\author{
Glenn C. Condie, Michelle F. Channon, Donald C. Craig, Mohan Bhadbhade, \\ Naresh Kumar, and David StC. Black*
}

School of Chemistry, UNSW Sydney, Sydney, NSW 2052, Australia

Email: d.black@unsw.edu.au

Dedicated to Professor Jan Bergman on the occasion of his $80^{\text {th }}$ birthday

Received 05-25-2020

Accepted 07-17-2020

Published on line $\quad 07-24-2020$

\section{Abstract}

This paper describes acid-catalysed reactions of 5,7-dimethoxy-1-methylindole and methyl 5,7-dimethoxyindole-2-carboxylate with a range of aldehydes and ketones. The former indole reacts selectively at C3, whereas the latter reacts preferentially at C4 but also at C3 depending on the reaction conditions. Reactions of indoles with 2,2-dimethoxypropane and triethyl orthoformate are also reported. A range of di- and triindolylmethanes are described, together with an indolo-triptycene of novel structure.
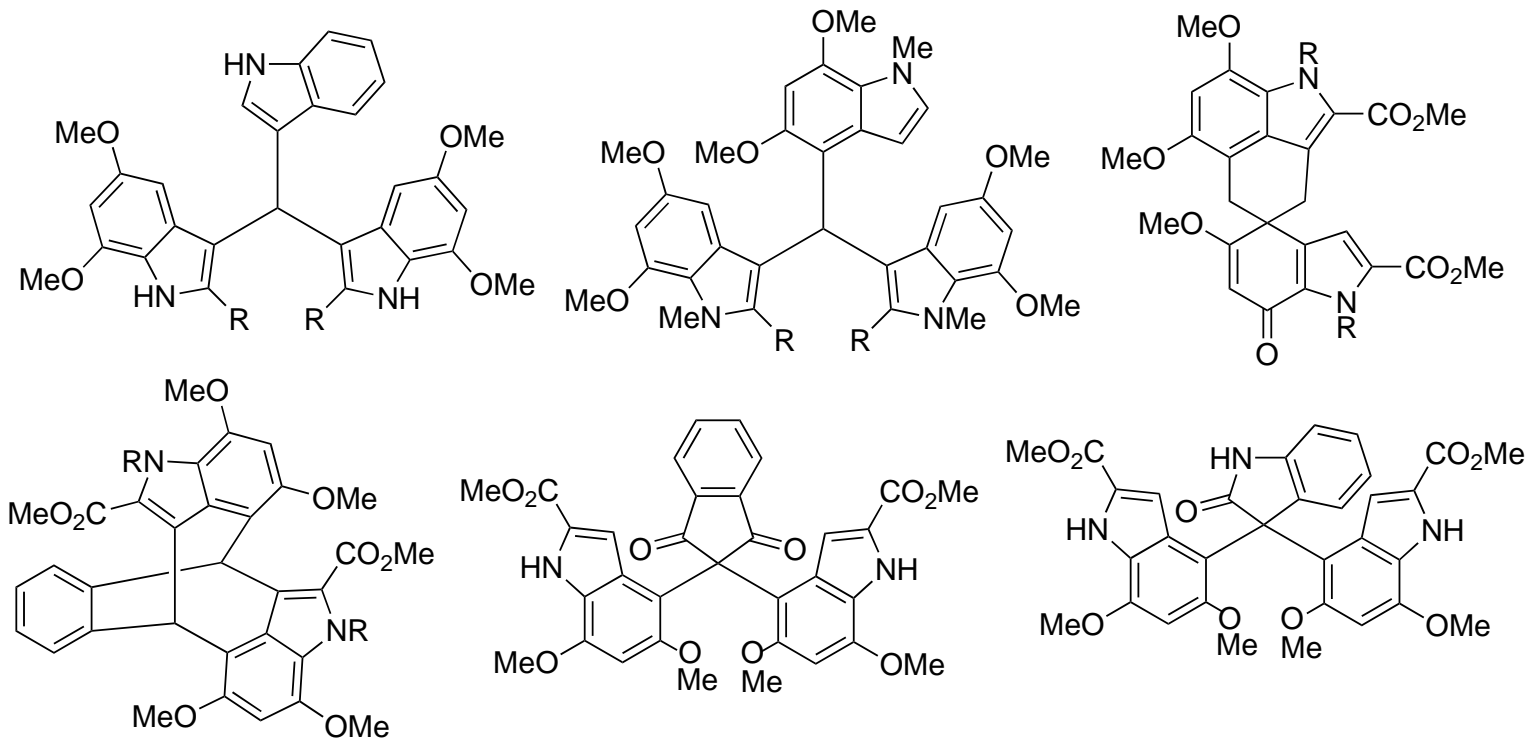

Keywords: Tri-indolylmethanes, di-indolylmethanes, indoles, benzindoles, triptycenes 


\section{Introduction}

In an attempt to modify the reactivity of indoles to provide diverse substitution in the benzene ring, we have carried out extensive investigations on 4,6-dimethoxyindoles, ${ }^{1-9}$ where the C7 position is strongly nucleophilic, and to a lesser extent on 5,7-dimethoxyindoles, ${ }^{10}$ where the $\mathrm{C} 4$ position is strongly nucleophilic. The reactivity patterns also depend on the presence of other substituents, and also the fact that the methoxy groups increase the general reactivity of the whole indole framework to the extent that favoured reactivity sites can also be positions $\mathrm{C} 2$ and $\mathrm{C} 3{ }^{11}$ We have previously reported a range of electrophilic substitution reactions on 5,7-dimethoxyindoles: ${ }^{10}$ these include formylation, acylation, bromination and nitration. Depending on the structure of the starting indole, reactions can take place at C4 or C3.

We now report a range of acid-catalysed reactions of several 5,7-dimethoxyindoles with aldehydes and ketones, with an emphasis on the comparative behaviour resulting from the structure of the starting material. ${ }^{10}$ The two main starting indoles chosen were 5,7-dimethoxy-1-methylindole 1 and methyl 5,7dimethoxyindole-2-carboxylate 5: the former in general tends to favour reactions at C3 and the latter at C4. The $\mathbf{N}$-methylindole $\mathbf{1}$ was chosen ahead of the simpler parent structure $\mathbf{2}$ because the electron-donating effect of the methyl group confers a more powerful nucleophilic reactivity at C3 (in the same way that $N$ methylindole is a more effective C3 nucleophile than indole). The presence of the electron-withdrawing carboxylic ester at C2 in indole $\mathbf{5}$ has the effect of reducing nucleophilic reactivity at C3 and therefore leading to preferential reaction at C4. Some related indoles 2-4, 6 and indole carbaldehydes 7-12 participate in some individual reactions (Figure 1). The syntheses of indoles 1 and $\mathbf{5}$ use different methodology, and all the indoles 1-11 have already been described. ${ }^{10}$ The indole 12 was prepared by the reaction of indole 11 with di-t-butyl) carbonate. Several aspects of the following work have been mentioned in the report of a conference lecture. ${ }^{12}$

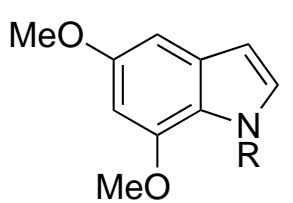

\begin{tabular}{|ll|}
\hline & $\mathrm{R}$ \\
\hline $\mathbf{1}$ & $\mathrm{Me}$ \\
$\mathbf{2}$ & $\mathrm{H}$ \\
$\mathbf{3}$ & $\mathrm{CH}_{2} \mathrm{Ph}$ \\
$\mathbf{4}$ & $\mathrm{COMe}$ \\
\hline
\end{tabular}
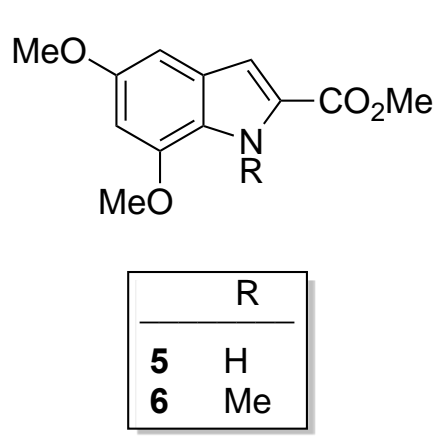
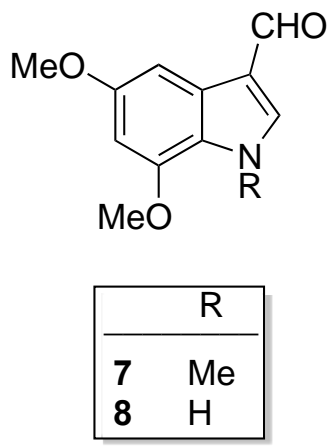

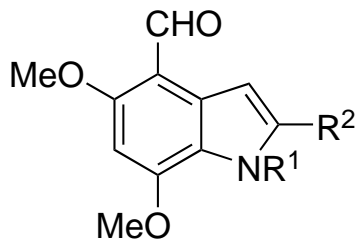

\begin{tabular}{|cll|}
\hline & $\mathrm{R}^{1}$ & $\mathrm{R}^{2}$ \\
\hline $\mathbf{9}$ & $\mathrm{Me}$ & $\mathrm{H}$ \\
$\mathbf{1 0}$ & $\mathrm{H}$ & $\mathrm{H}$ \\
11 & $\mathrm{H}$ & $\mathrm{CO}_{2} \mathrm{Me}$ \\
12 & $\mathrm{Boc}$ & $\mathrm{CO}_{2} \mathrm{Me}$ \\
\hline
\end{tabular}

Figure 1. Selected 5,7-dimethoxyindole starting materials.

\section{Results and Discussion}

\section{Reactions with formaldehyde}

The $N$-methylindole 1 failed to react with formaldehyde at room temperature but a complex mixture of products formed when the reaction mixture was heated. However, the less reactive 5,7-dimethoxy-1methylindole-3-carbaldehyde 7 reacted smoothly with formaldehyde in glacial acetic acid at room temperature to give the $4,4^{\prime}$-diindolylmethane 13 in $66 \%$ yield. The $N$-acetylindole 4 also combined with 
formaldehyde in glacial acetic acid to give the 4,4'-diindolylmethane 14 in $57 \%$ yield (Scheme 1). Presumably the C3 position is deactivated by the electron-withdrawing acetyl group. On the other hand, 5,7dimethoxyindole-4-carbaldehyde $\mathbf{1 0}$ and also its $\mathbf{N}$-methyl derivative $\mathbf{9}$ gave complex mixtures under the same reaction conditions.<smiles>COc1cc(OC)c2c(c1)c(C=O)cn2C</smiles>

7<smiles>COc1cc(OC)c2c(ccn2C(C)=O)c1</smiles>

4
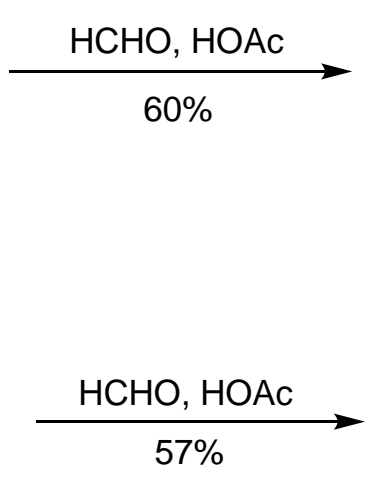<smiles></smiles>

13<smiles>COCn1ccc2c(Cc3c(OC)cc(OC)c4c3ccn4C(C)C)c(OC)cc(OC)c21</smiles>

14

Scheme 1. Formation of 4,4'-diindolylmethanes 13 and 14.

The indole-2-carboxylate 5 reacted slowly with formaldehyde in acetic acid over several days to give the 4,4'-diindolylmethane 15 in $85 \%$ yield (Scheme 2). An attempt to achieve a faster reaction using methanolic hydrochloric acid gave a yield of only $27 \%$. The structures of the new $4,4^{\prime}$-diindolylmethanes 13,14 and 15 were clear from their NMR spectra, and the linking methylene protons resonated at 4.99, 4.30 and $4.40 \mathrm{ppm}$ respectively, and the linking carbon atoms resonated at 29.0, 22.4 and 22.6 respectively.

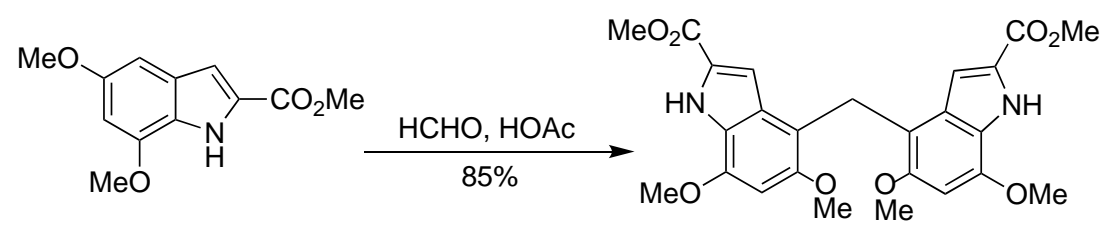

5<smiles>COc1cc(OC)c2[nH]c(C(C)=O)cc2c1CO</smiles>

16<smiles>COc1cc(OC)c2[nH]c(C(C)=O)cc2c1C=O</smiles>

12
15

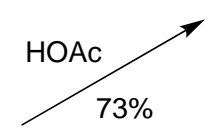

18

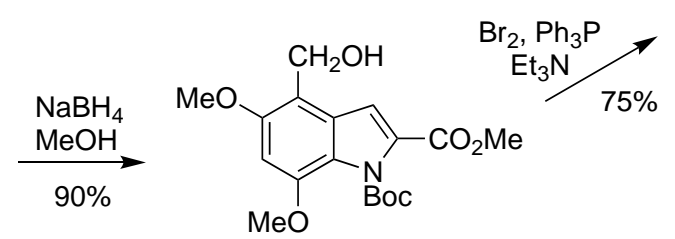

17

Scheme 2. Formation of 4,4'-diindolylmethanes 15 and 18. 
In any acid-catalysed addition of an indole to formaldehyde the initial intermediate would be a hydroxymethyl derivative. Therefore the indole-4-carbaldehyde $\mathbf{1 0}$ was reduced by sodium borohydride to give the 4-hydroxymethylindole 16 in 77\% yield: treatment of this compound with acetic acid overnight gave a $73 \%$ yield of the 4,4'-diindolylmethane 15. The related 1-butyloxycarbonyl-4-hydroxymethylindole 17 was prepared by sodium borohydride reduction of the 1-butyloxycarbonylindole-4-carbaldehyde 12 , and in an attempt to convert this by a standard procedure ${ }^{13-14}$ into the related 4-bromomethyl derivative, instead it gave the 1-butyloxycarbonyl-4,4'-diindolylmethane 18 in 75\% yield (Scheme 2). The structure was confirmed by almost quantitative conversion of the diindolylmethane $\mathbf{1 5}$ into compound $\mathbf{1 8}$ by reaction with di-t-butyl carbonate in acetonitrile. The precise mechanism for the formation of the diindolylmethane 18 is not clear. Presumably the intermediate bromomethyl compound undergoes further combination with the indole 17. However, many cases have been reported where 7-hydroxymethyl-4,6-dimethoxyindoles undergo acidcatalysed ipso-substitution reactions with elimination of formaldehyde to give 7,7'-diindolylmethanes, $8,9,15$ (e.g. the conversion of indole 16 to diindolylmethane 15) so the two 4-hydroxymethylindoles 16 and 17 were further investigated. When 4-hydroxymethylindole 16 was stirred in methanol with concentrated hydrochloric acid at room temperature, the spiro-dienone 19 precipitated out as a highly pure white solid in $91 \%$ yield (Scheme 3).
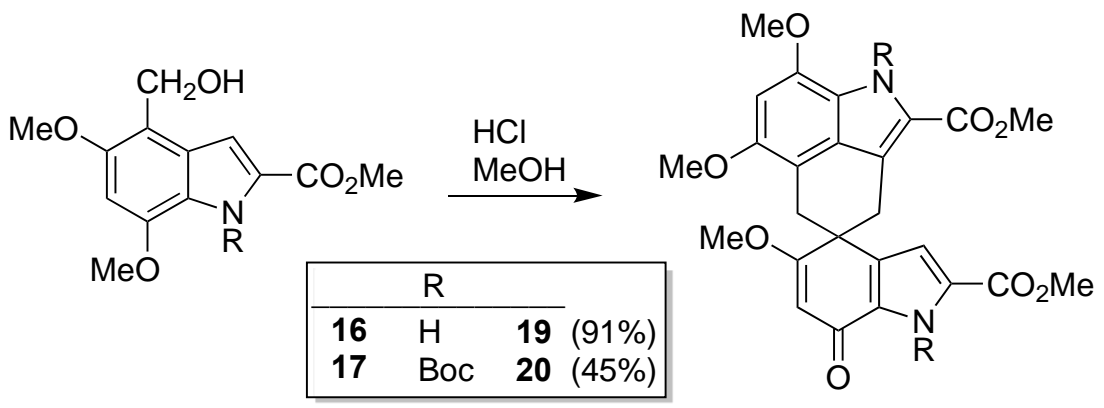<smiles></smiles>

$21(12 \%)$

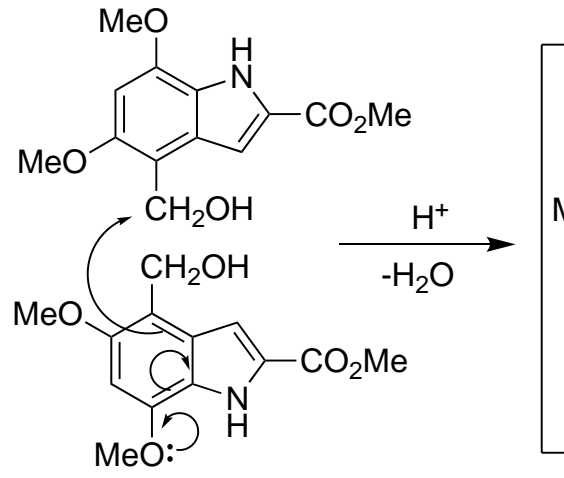

$2 \times 16$

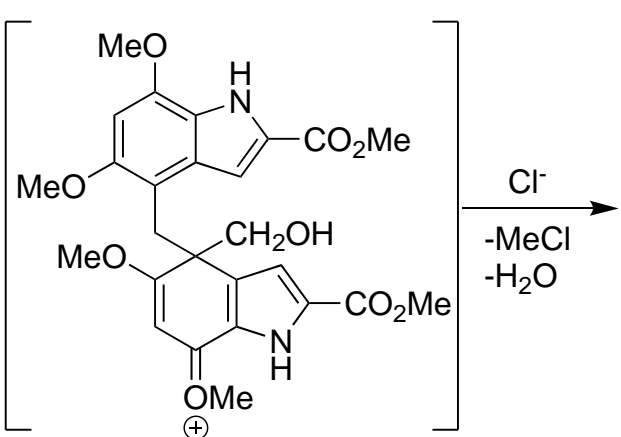

22<smiles>COC(=O)c1cc2c([nH]1)C(=O)C=C(OC)C21Cc2c(OC)cc(OC)c3[nH]c(C(C)=O)c(c23)C1</smiles>

19

Scheme 3. Formation of indolo-spiro-dienones 19 and 20.

A similar reaction with indole 17 gave a mixture of the spiro-dienone 20 (45\%), the 4,4'-diindolylmethane $18(30 \%)$, and the 4-methoxymethylindole 21 (12\%). The structure of the dienone 19 was established by extensive 1D and 2D NMR spectroscopy: a suitable crystal for X-ray structure determination could not be obtained. The ${ }^{1} \mathrm{H}$ NMR spectrum showed the presence of two NH resonances and only five methoxy proton resonances, suggesting that the compound contains two indole moieties but that one methoxy group has been lost. The ${ }^{13} \mathrm{C}$ NMR spectrum showed methoxy carbon resonances between 52 and 58 ppm representing 
the methoxy and ester groups. A resonance for a quaternary carbon at $44.2 \mathrm{ppm}$ indicated the spiro-structure and a resonance for the quinone-type carbonyl carbon appeared at $181.0 \mathrm{ppm}$. The proposed mechanism for the formation of the dienone $\mathbf{1 9}$ initially involves the electrophilic addition of a hydroxymethylene group of one molecule of indole $\mathbf{1 6}$ to the substituted C4 position of another molecule of indole $\mathbf{1 6}$ to give the C4 disubstituted indole 22. While this intermediate might be expected to lose formaldehyde to give the diindolylmethane 15, under these conditions electrophilic substitution occurs at the indole C3 to form the sixmembered ring of the product 19. (Scheme 3).

\section{Reactions with aryl- and heteroaryl-aldehydes}

The 5,7-dimethoxyindoles 1 and $\mathbf{5}$ react readily with aryl aldehydes under acidic conditions to form diindolylmethanes. The most successful conditions involve the use of glacial acetic acid or methanolic hydrochloric acid. Indole 1 combined with a range of benzaldehydes in glacial acetic acid at room temperature to give the aryl-3,3'-diindolylmethanes 23-26 in 66-91\% yields (Scheme 4).

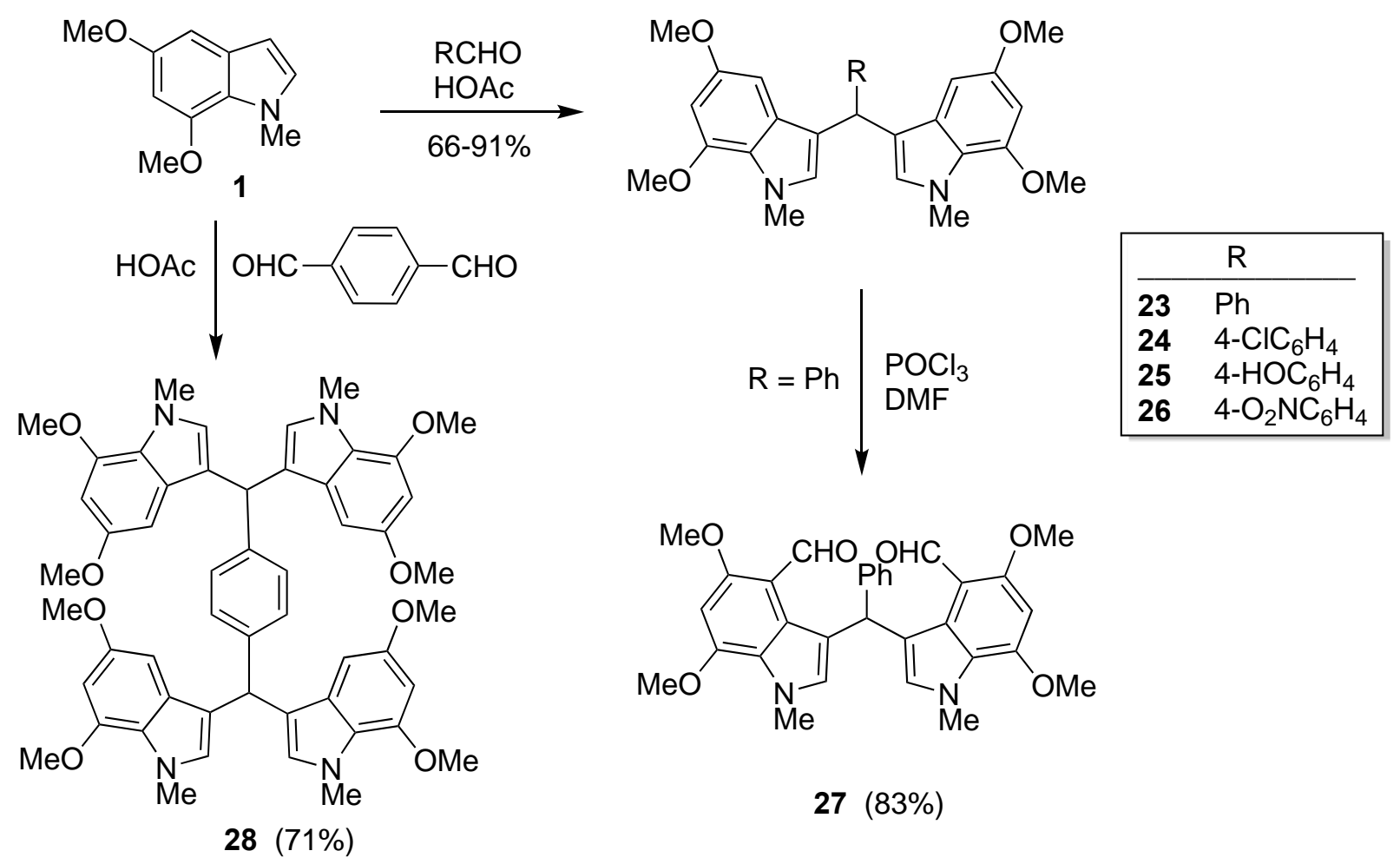

Scheme 4. Formation of aryl-3,3'-diindolylmethanes 23-28.

The presence of methoxy groups at $\mathrm{C} 5$ and $\mathrm{C} 7$ did not change the normal regiochemistry for the formation of diindolylmethanes. ${ }^{16,17}$ The bond formation at $\mathrm{C} 3$ was established by ${ }^{1} \mathrm{H}$ NMR data showing the metacoupling between $\mathrm{H} 4$ and H6. The diindolylmethane $\mathbf{2 3}$ was also converted to the 4,4'-dicarbaldehyde $\mathbf{2 7}$ under Vilsmeier reaction conditions. Indole $\mathbf{1}$ also reacted with terephthaldialdehyde to yield the tetraindolyldimethane $\mathbf{2 8}$ in $\mathbf{7 1 \%}$ yield. In contrast, the indole $\mathbf{5}$ combined with $p$-chloro- and $p$-methoxy-benzaldehydes under the improved conditions of concentrated hydrochloric acid in methanol to give the aryl-4,4'diindolylmethanes 29-30, which precipitated out of solution in high yields (Scheme 5). Trace amounts of the more soluble 3,4'-diindolylmethane isomers were detected but not isolated and characterized. When indole 5 was reacted with $p$-chlorobenzaldehyde under the more powerful conditions of phosphoryl chloride in chloroform, a complex mixture of products was formed: after extensive chromatography only the 3,4'- 
diindolylmethane $\mathbf{3 1}$ could be isolated in 37\% yield and characterized. The $N$-methyl analog $\mathbf{6}$ of the indole $\mathbf{5}$ underwent the same reaction to give the 4,4'-diindolylmethane $\mathbf{3 2}$ in $93 \%$ yield. The diindolylmethane 29 was also converted to the $N$-Boc derivative 33 by reaction with di-( $t$-butyl) carbonate (Scheme 5$)$.

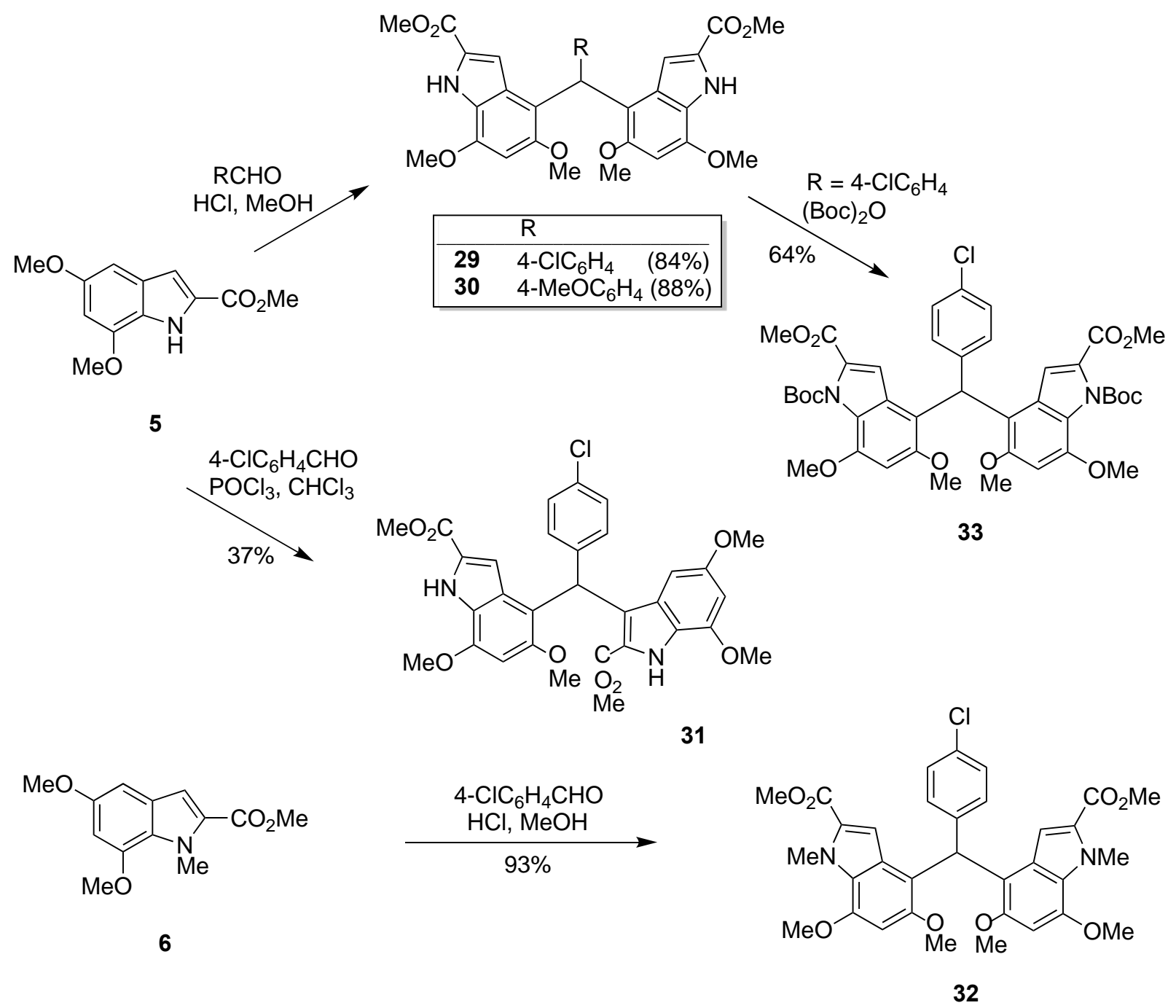

Scheme 5. Formation of aryl-4,4'-diindolylmethanes 29, 30, 32, 33 and aryl-3,4'-diindolylmethane 31.

In an attempt to synthesise tri-indolylmethanes, the indoles $\mathbf{1}$ and $\mathbf{5}$ were reacted with several indole aldehydes. Indole 1 reacted in glacial acetic acid with indole-3-carbaldehyde 34 to give the 3,3',3'triindolylmethane 35 in only $25 \%$ yield. Slightly better yields of $37 \%$ and $47 \%$ were obtained in reactions with the 5,7-dimethoxyindole-4-carbaldehyde 10 and the $N$-methyl-3-carbaldehyde 7 to give the 3,3',4"triindolylmethane $\mathbf{3 6}$ and the 3,3',3'-triindolylmethane $\mathbf{3 7}$ respectively. However, since compound $\mathbf{3 7}$ is completely symmetrical, its yield was dramatically increased to $88 \%$ by the reaction of indole 1 with triethyl orthoformate in methanol in the presence of a catalytic amount of $p$-toluenesufonic acid. ${ }^{18-24}$ This reaction was more generally applied to the $\mathrm{NH}$-indole 2 and the $\mathrm{N}$-benzylindole $\mathbf{3}$ which yielded the respective 3,3'.3"triindolylmethanes 38 and $\mathbf{3 9}$ in $37 \%$ and $77 \%$ yields (Scheme 6). 


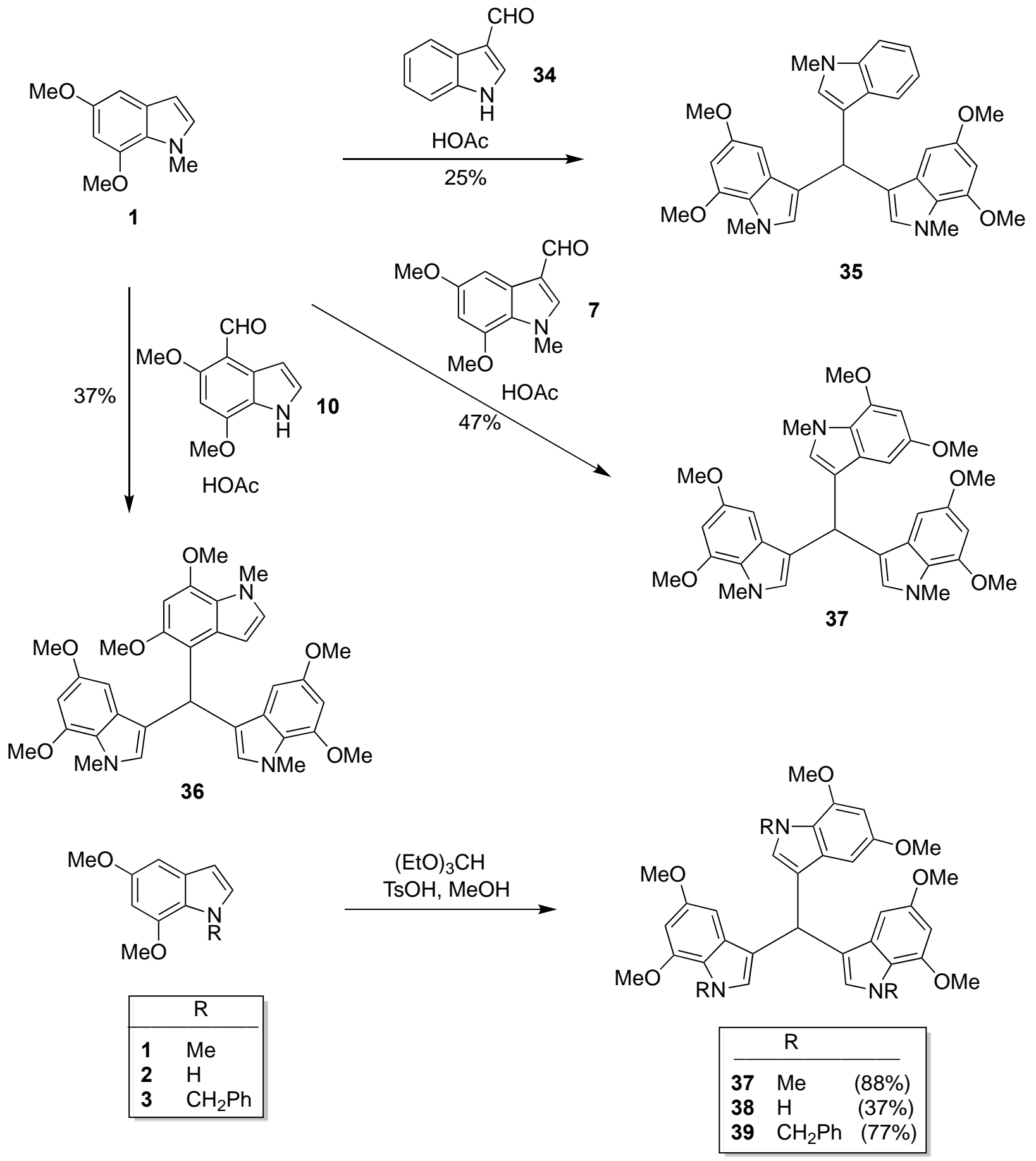

Scheme 6. Formation of 3,3',3'-triindolylmethanes $35,37-39$ and 3,3',4'-triindolylmethane 36 .

Condensation of indole 5 with indole-3-carbaldehyde 34 in methanolic hydrochloric acid gave the 3,3',3"triindolylmethane $\mathbf{4 0}$ in $46 \%$ yield, and the corresponding reaction with the methyl 5,7-dimethoxyindole-4carbaldehyde 11 gave an $83 \%$ yield of a mixture of the 3,3',4"-triindolylmethane 41 and the 3,3',3"triindolylmethane $\mathbf{4 2}$ in a ratio of 88:12. This result contrasts with the reaction of indole 5 with 4chlorobenzaldehyde under the same conditions, which showed selective reaction at C4 (see Scheme 5). Presumably the greater steric bulk of the indole aldehydes $\mathbf{3 4}$ and $\mathbf{1 1}$ could be a factor in directing the reaction away from C4 to the more accessible C3. In comparison, the condensation of indole $\mathbf{5}$ with triethyl orthoformate gave an $80 \%$ yield of a mixture of the $3,3^{\prime}, 4^{\prime \prime}$-triindolylmethane $\mathbf{4 1}$ and the $3,3^{\prime}, 3^{\prime \prime}$ - 
triindolylmethane $\mathbf{4 2}$ in a ratio of 98:2 (Scheme 7). The minor product $\mathbf{4 2}$ presumably arises as a result of reversible steps involved in the formation of the triindolylmethanes. Several triindolylmethanes have been reported as natural products from a marine bacterium ${ }^{25}$ and also as products from reactions of indole with $N$ methylindol-2-one or 1,3-dimethylimidazolidin-2-one and phosphoryl chloride. ${ }^{26}$<smiles>COC(=O)c1cc2cc(OC)cc(OC)c2[nH]1</smiles>

5<smiles>COC(=O)c1cc2cc(OC)cc(OC)c2[nH]1</smiles>

5

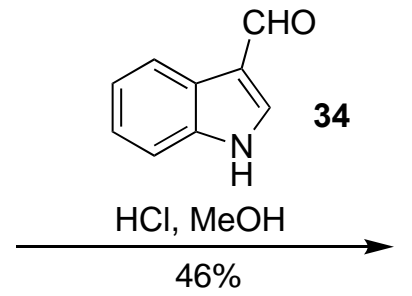<smiles>COc1cc(OC)c2[nH]c(C(C)=O)cc2c1C=O</smiles>

$\mathrm{HCl}, \mathrm{MeOH}$

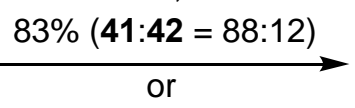

$(\mathrm{EtO})_{3} \mathrm{CH}$

$\mathrm{TsOH}, \mathrm{MeOH}$

$80 \%(41: 42=98: 2)$<smiles></smiles>

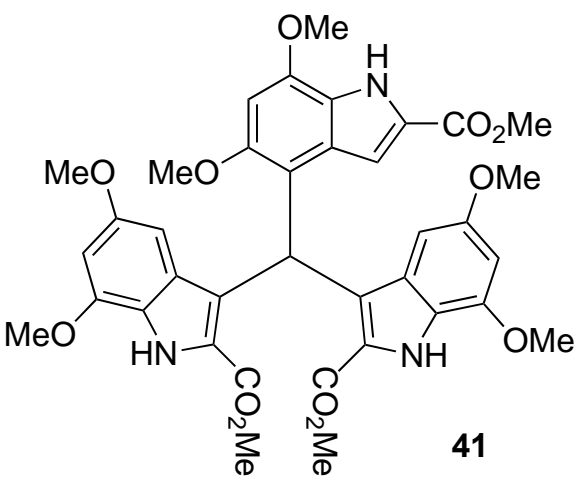<smiles>COC(=O)c1c(C(c2c([O-])[nH]c3c(OC)cc(OC)cc23)c2c([O-])[nH]c3c(OC)cc(OC)cc23)[nH]c2c(OC)cc(OC)cc12</smiles>

Scheme 7. Formation of 3,3',4"-triindolylmethane $\mathbf{4 1}$ and 3,3',3'-triindolylmethanes $\mathbf{4 0}$ and 42.

\section{Reactions with o-phthaldialdehyde}

o-Phthaldialdehyde represents a special case in reactions of aldehydes with indoles. While terephthaldialdehyde behaves normally, o-phthaldialdehyde has the possibility to react at two adjacent positions of an indole if they are available. We have previously shown that 1-methyl-4,6-dimethoxyindole reacts regioselectively at C2 and C3 with 0 -phthaldialdehyde to give isomeric indolylbenzocarbazoles depending on the conditions. ${ }^{27}$ The indole 1 has positions C2, C3 and C4 available, so it was reacted with o-phthaldialdehyde in glacial acetic acid and found to give the 6-(3-indolyl)benzocarbazole $\mathbf{4 3}$ in $34 \%$ yield (Scheme 8 ). This is consistent with a slow reaction to give the thermodynamically more stable product, as previously described. ${ }^{27}$ 
On the other hand, indole 5 only has availability for reaction at C3 and C4: it combined with ophthaldialdehyde in methanolic hydrochloric acid to give a very pure white precipitate of the heterotriptycene $\mathbf{4 4}$ in $90 \%$ yield (Scheme 8). Similarly high yields were obtained when the reaction was carried out in either phosphoryl chloride or $p$-toluenesulfonic acid.<smiles>COc1cc(OC)c2c(ccn2C)c1</smiles>

1<smiles>COc1cc(OC)c2[nH]c(C(C)=O)cc2c1</smiles>
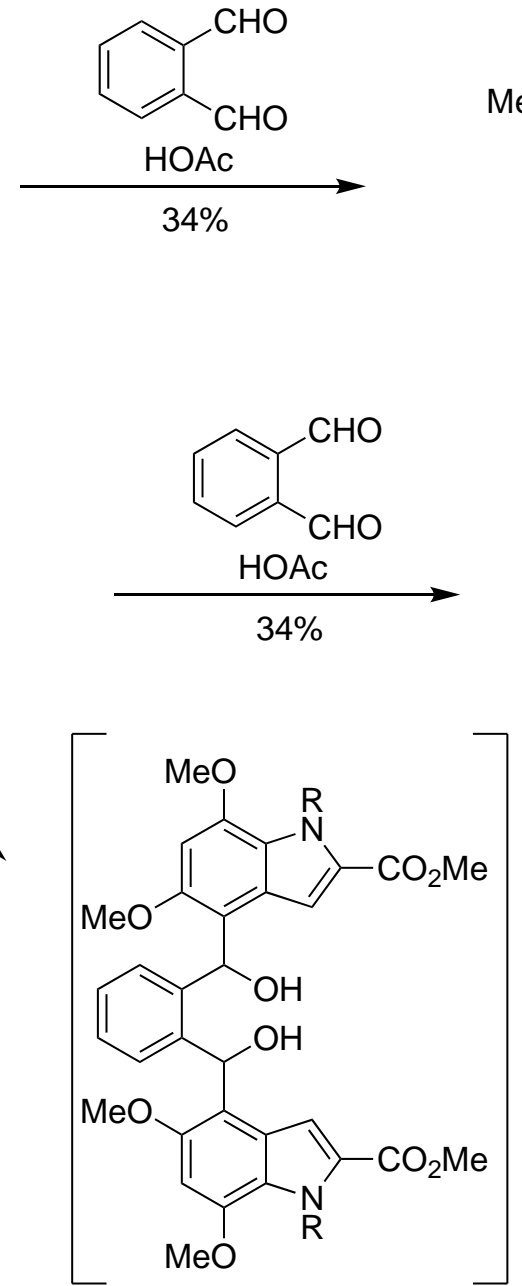

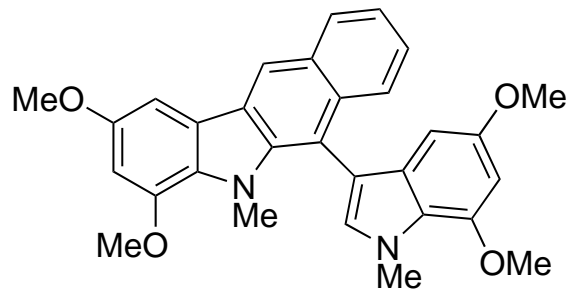

43

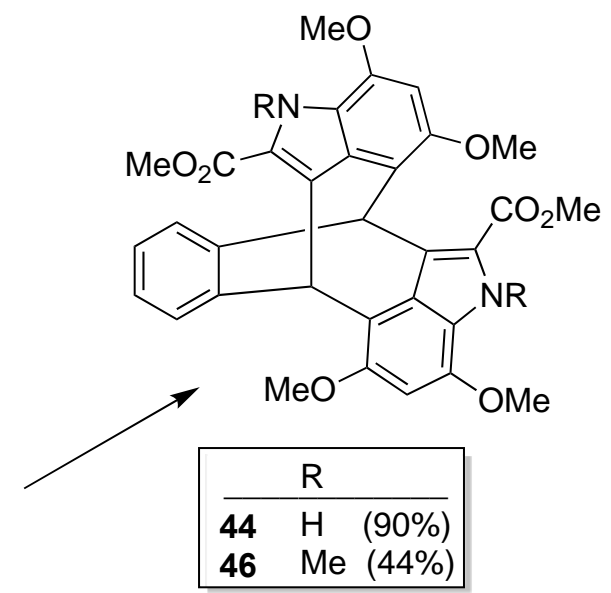

45

Scheme 8. Formation of indolobenzocarbazole 43 and indolotriptycenes 44 and 46.

The structure of compound $\mathbf{4 4}$ was established by extensive NMR spectroscopy and also by an X-ray crystal structure (Figure 2). The ${ }^{1} \mathrm{H}$ NMR spectrum showed only one $\mathrm{NH}$, one $\mathrm{H} 6$ and three methoxy proton resonances, indicating that the two indole rings were in the same environment. A singlet at $6.90 \mathrm{ppm}$ was assigned to the two bridging methines, thus establishing the presence of two 3,7'-diindolylmethane links, rather than the alternative possibility of one 3,3'-link and one $7,7^{\prime}$-link. The suggested mechanism proceeds via the intermediate $\mathbf{4 5}$ as a result of indole C7 attack on each formyl group (Scheme 8). The $\mathrm{N}$-methylindole 6 also reacted with 0 -phthaldialdehyde to give the $N$-methylated triptycene $\mathbf{4 6}$ in $44 \%$ yield. The lower yield is thought to be a function of the greater solubility of the product, and no attempt was made to obtain a second crop from the filtrate after collecting the product. The characteristic triptycene structure has been put to use in a variety of applications, including materials chemistry, host-guest chemistry, and molecular motors. ${ }^{28}$ 


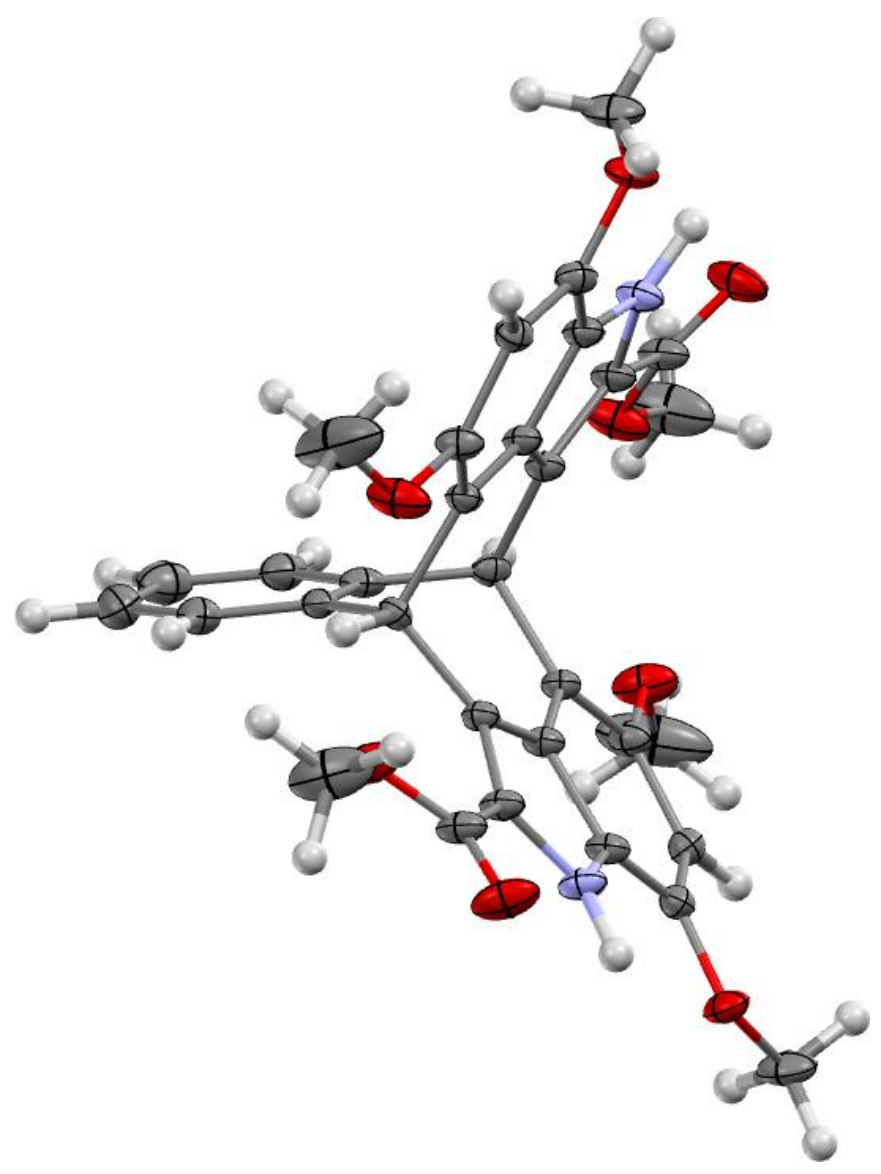

Figure 2. ORTEP diagram of compound 44.

\section{Reactions with ketones}

Neither indole $\mathbf{1}$ nor $\mathbf{5}$ underwent any reaction with a range of acetophenones. The $N$-methylindole $\mathbf{1}$ underwent reaction with acetone and acetic anhydride to give the 3,3'-diindolylmethane 47 in a yield of $18 \%$. When glacial acetic acid was used as the solvent an even lower yield (10\%) of the benzo[cd]indole 48 was obtained from a complex mixture. The acid-catalysed condensation of indole and acetone is known to give rise to multiple products. ${ }^{17,29}$ Indole 5 failed to react with acetone, but it did react when heated in 2,2dimethoxypropane in the presence of a catalytic amount of $p$-toluenesulfonic acid to give the benzo[cd]indole 49 in 34\% yield (Scheme 9). The two benzoindoles 48 and 49 show different methyl substitution patterns, indicating that the initial attack for indole $\mathbf{1}$ takes place from C3, while that for indole $\mathbf{5}$ takes place from C4. The mechanism appears to be a stepwise intramolecular aldol-type, because neither indole $\mathbf{1}$ nor $\mathbf{5}$ showed any reaction with mesityl oxide. The structures of benzindoles 48 and 49 were established by extensive 1D and 2D NMR experiments. Other benz $[c d]$ indole structures have been reported in similar reactions. ${ }^{30}$ 
<smiles>COc1cc(OC)c2c(ccn2C)c1</smiles><smiles>COc1cc(OC)c2[nH]c(C(C)=O)cc2c1</smiles>

5<smiles></smiles>

47<smiles>COc1cc(OC)c2c3c1Cc1c(C(C)=O)[nH]c(c1-3)C(C)=CC2(C)C</smiles>

49

Scheme 9. Formation of benz $[c d]$ indoles 48 and 49.

The indole 5 underwent reaction with two reactive ketones, ninhydrin and 1-pentanoylisatin. When indole $\mathbf{5}$ and ninhydrin were heated together in methanol with concentrated hydrochloric acid a yellow precipitate of the 1:1 adduct $\mathbf{5 0}$ was obtained in $91 \%$ yield, regardless of the stoichiometry. However, when<smiles>COc1cc(OC)c2[nH]c(C(C)=O)cc2c1</smiles>

5<smiles>O=C1c2ccccc2C(=O)C1(O)O</smiles>

$\mathrm{HCl}, \mathrm{MeOH}$

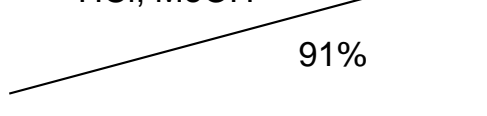<smiles>COc1cc(OC)c2[nH]c(C(C)=O)cc2c1C1(c2c(OC)cc(OC)c3[nH]c(C(C)=O)cc23)C(=O)c2ccccc2C1=O</smiles>

$\mathrm{TsOH}$, toluene<smiles>COc1cc(OC)c2[nH]c(C(C)=O)cc2c1C1(O)C(=O)c2ccccc2C1=O</smiles><smiles>C[13C](=O)c1ccccc1C(=O)O</smiles>

Scheme 10. Formation of 2-(4-indolyl)indane-1,3-dione 50 and 2,2-di(4-indolyl)indane-1,3-dione 51. 
two equivalents of indole $\mathbf{5}$ and one of ninhydrin were heated together in toluene with a catalytic amount of p-toluenesulfonic acid, initial formation of the adduct $\mathbf{5 0}$ was observed, but continued heating for two days showed that this was converted into the 4,4'-diindolylmethane 51 in $98 \%$ yield (Scheme 10).

Indole $\mathbf{5}$ failed to undergo any reaction with isatin but it reacted with the more reactive 1-pentanoylisatin 52 when heated in methanol with concentrated hydrochloric acid to give the 4,4'-diindolylmethane $\mathbf{5 3}$ in $\mathbf{7 5 \%}$ yield (Scheme 11). Under these conditions it is known ${ }^{31,32}$ that the 1-pentanoylisatin could undergo ringopening following attack at C2 by methanol to give the related methyl glyoxylate 54, which could then react with the indole 5. Subsequent hydrolysis of the amide and cyclisation, for which there is precedent, ${ }^{33}$ would then generate the observed product 53. This possibility was checked and it was found that compound $\mathbf{5 3}$ was obtained in $79 \%$ yield when indole $\mathbf{5}$ and the methyl glyoxylate $\mathbf{5 4}$ were reacted together under the same conditions (Scheme 11). Some 3,3-diindolylindolin-2-ones are known where the indoles are linked through C3 and also C2: these compounds show interesting biological activity. ${ }^{34}$

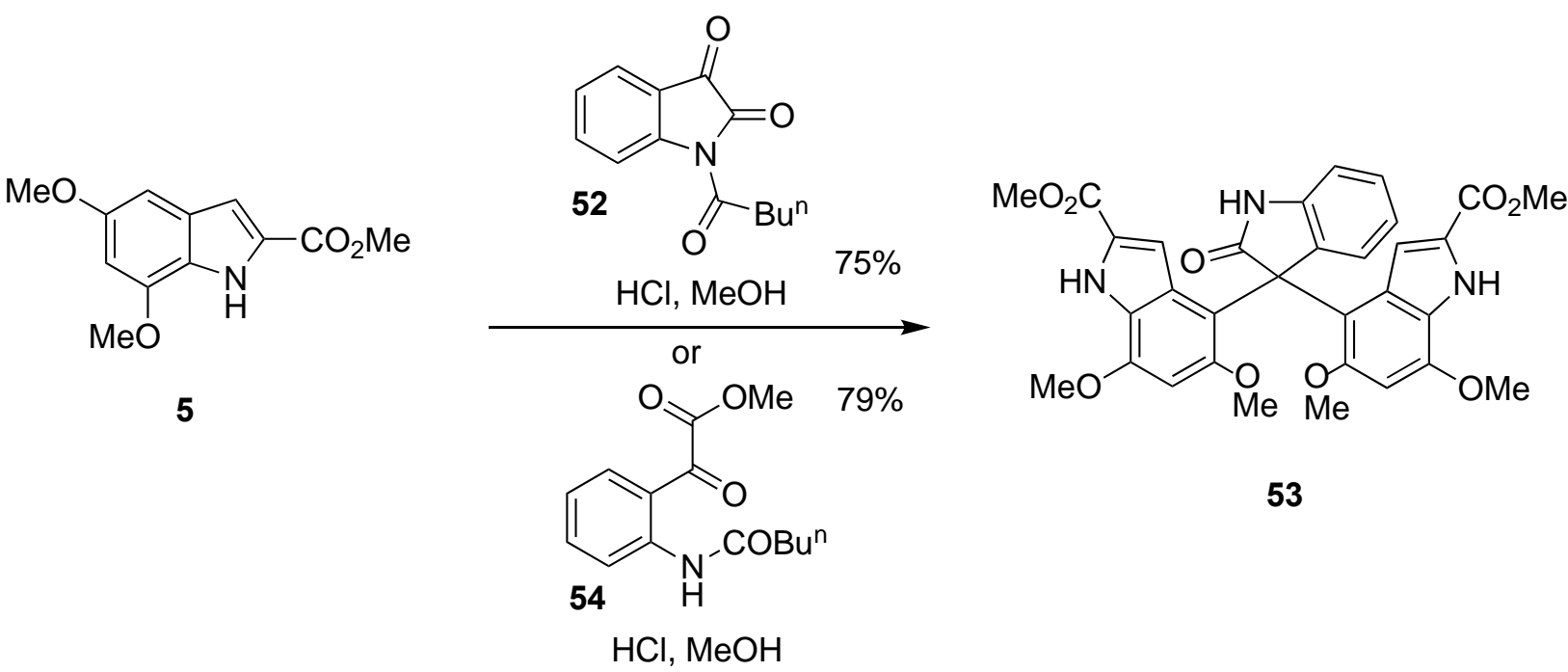

Scheme 11. Formation of 3,3-di(4-indolyl)oxindole 53.

\section{Conclusions}

A wide range of acid-catalysed reactions of 5,7-dimethoxyindoles with aldehydes and ketones has established many high yielding syntheses of diindolyl- and triindolyl-methanes, with linkages from C3 and C4. The reactions are most successful with formaldehyde, aryl aldehydes and highly reactive ketones. Aryl aldehydes give rise to aryldiindolylmethanes, while reactions with indole aldehydes yield triindolylmethanes. Reactions with $o$-phthalaldehyde generate triptycene analogs of novel structure. Unusual spiro-dienones can also be formed by double reaction at $\mathrm{C} 4$, and reactions with acetone give benzo[ $c d]$ indoles, although only in modest yield.

In summary: the positioning of methoxy groups at $\mathrm{C} 5$ and $\mathrm{C} 7$ on the indole framework greatly expands the synthetic potential of indole reactivity. 


\section{Experimental Section}

General. ${ }^{1} \mathrm{H}$ and ${ }^{13} \mathrm{C}$ NMR spectra were recorded on a Bruker AC300F $\left({ }^{1} \mathrm{H}: 300 \mathrm{MHz},{ }^{13} \mathrm{C}: 75.5 \mathrm{MHz}\right)$ or a Bruker AM500 spectrometer. The chemical shifts $(\delta)$ and coupling constants $(J)$ are expressed in ppm and hertz respectively. Carbon attribution $\mathrm{C}, \mathrm{CH}, \mathrm{CH}_{2}$ and $\mathrm{CH}_{3}$ were determined by ${ }^{13} \mathrm{C}$, DEPT and $\mathrm{HMQC}$ experiments. Infrared (IR) spectra were recorded on a Mattson Genesis Series FTIR spectrometer using potassium bromide disks, except where specified. Ultraviolet and visible (UV/Vis) spectra were recorded in tetrahydrofuran or methanol using a Carey 100 spectrometer. Mass spectra were recorded on a VG Quattro MS (EI) or a Finnigan MAT (MALDI). High resolution mass spectrometry (HRMS) was carried out at the Research School of Chemistry, Australian National University. Melting points were measured using a Mel-Temp melting point apparatus. Microanalyses were performed at the Campbell Microanalytical Laboratory, University of Otago, New Zealand. Column chromatography was carried out using Merck 230-400 mesh silica gel or Merck 70-230 mesh silica gel, whilst preparative TLC was performed using Merck $60 \mathrm{GF}_{254}$ silica gel. X-ray crystallography was conducted with a suitable single crystal and crystallographic data excluding structure factors have been deposited with the Cambridge Crystallographic Data Centre: Compound 44 - Deposition Number 2004528

Methyl 1-(tert-butyloxycarbonyl)-4-formyl-5,7-dimethoxyindole-2-carboxylate (12). A suspension of methyl 4-formyl-5,7-dimethoxyindole-2-carboxylate (11) $(0.49 \mathrm{~g}, 1.85 \mathrm{mmol})$, di-tert-butyl dicarbonate $(0.79 \mathrm{~g}, 3.62$ $\mathrm{mmol})$ and $\mathrm{N}, \mathrm{N}$-dimethylaminopyridine $(48 \mathrm{mg})$ in anhydrous acetonitrile $(12.0 \mathrm{~mL})$ was stirred at room temperature, under nitrogen, for $70 \mathrm{~min}$. The solvent was then evaporated in vacuo and the remaining residue purified via suction column chromatography (dichloromethane) to give the title compound ( $0.66 \mathrm{~g}$, $98 \%)$ as a colourless syrup that solidified upon standing, $\mathrm{mp} 164-166{ }^{\circ} \mathrm{C} . \mathrm{IR}\left(\mathrm{V}_{\max }, \mathrm{cm}^{-1}\right): 1755,1714,1661$, 1585, 1399, 1367, 1233, 1156, 1108. UV/Vis $\left(\lambda_{\max }, \mathrm{nm}, \varepsilon, \mathrm{cm}^{-1} \mathrm{M}^{-1}\right): 242(21,600), 267(12,000), 321(18,500)$, 354 (11,700), 369 (8,300); ${ }^{1} \mathrm{H}$ NMR (300 MHz, $\left.\mathrm{CDCl}_{3}\right): \delta_{\mathrm{H}} 1.64\left(9 \mathrm{H}, \mathrm{s}, \mathrm{CMe}_{3}\right), 3.90,3.94,3.99$ (each $\left.3 \mathrm{H}, \mathrm{s}, \mathrm{OMe}\right)$, $6.42(1 \mathrm{H}, \mathrm{s}, \mathrm{H} 6), 7.98(1 \mathrm{H}, \mathrm{s}, \mathrm{H} 3), 10.49(1 \mathrm{H}, \mathrm{s}, \mathrm{CHO}) .{ }^{13} \mathrm{C} \mathrm{NMR}\left(75 \mathrm{MHz}, \mathrm{CDCl}_{3}\right): \delta_{\mathrm{c}} 27.8\left(\mathrm{CMe}_{3}\right), 52.5,57.4,56.2$ (OMe), 85.6 (밀 $), 93.2$ (C6), 112.0 (C3), 111.1, 123.3, 126.4, 130.5, 150.6, 153.2 (aryl C), 162.8 and 161.4 (CO and $\mathrm{CO}_{2} \mathrm{Me}$ ), 188.8 (CHO). MS (+El, $\left.\mathrm{m} / \mathrm{z}, \%\right): 363$ (M, 5), 290 (14), 264 (12), 263 (100), 232 (20), 231 (97), 230 (32), 214 (13), 204 (17), 203 (11), 202 (63), 188 (13), 174 (22), 160 (17), 144 (11). Anal. calcd for $\mathrm{C}_{18} \mathrm{H}_{21} \mathrm{NO}_{7}$ : C, 59.5; H, 5.8; N, 3.8. Found: C, 59.5; H, 5.8; N, 3.6\%.

4,4'-Bis(5,7-dimethoxy-1-methylindolyl)methane-3,3'-dicarbaldehyde (13). 5,7-Dimethoxy-1-methylindole-3carbaldehyde (7) $(0.15 \mathrm{~g}, 0.69 \mathrm{mmol})$ was dissolved in glacial acetic acid $(3 \mathrm{~mL})$ and the reaction mixture was put under an inert gas atmosphere. Aqueous formaldehyde solution $(37 \%, 0.04 \mathrm{~mL}, 0.54 \mathrm{mmol})$ was added, and the reaction mixture was stirred at room temperature for $6 \mathrm{~h}$. The resulting precipitate was filtered, washed with water and dried, affording 4,4'-di(5,7-dimethoxy-1-methylindolyl)methane-3,3'-dicarbaldehyde $13(0.10 \mathrm{~g}, 66 \%)$ as a white solid, $\mathrm{mp} 294-295^{\circ} \mathrm{C}$. IR $\left(\mathrm{vmax}, \mathrm{cm}^{-1}\right): 1643,1584,1528,1496,1453,1415,1315$, 1245, 1210, 1119, 1062. UV/Vis $\left(\lambda \max , \mathrm{nm}, \varepsilon, \mathrm{cm}^{-1} \mathrm{M}^{-1}\right)$ : $257(24,000), 270(19,000), 314(13,000), 333$ $(14,000) .{ }^{1} \mathrm{H}$ NMR $\left(300 \mathrm{MHz}, \mathrm{CDCl}_{3}\right): \delta_{H} 3.43,3.84$ and $4.04\left(18 \mathrm{H}, \mathrm{s}, \mathrm{OMe}\right.$ and NMe), $4.99\left(2 \mathrm{H}, \mathrm{s}, \mathrm{CH}_{2}\right), 6.34$ $(2 \mathrm{H}, \mathrm{s}, \mathrm{H} 6), 7.72(2 \mathrm{H}, \mathrm{s}, \mathrm{H} 2), 10.36(2 \mathrm{H}, \mathrm{s}, \mathrm{CHO}) .{ }^{13} \mathrm{C} \mathrm{NMR}\left(75 \mathrm{MHz}, \mathrm{CDCl}_{3}\right): \delta_{\mathrm{c}} 29.0\left(\mathrm{CH}_{2}\right), 37.9(\mathrm{C} \times 2, \mathrm{NMe})$, 55.6 and 57.1 ( $\mathrm{C} \times 4$, OMe), $93.7(\mathrm{C} \times 2$, aryl $\mathrm{CH}), 115.9,119.5,123.1,128.8,(\mathrm{C} \times 8$, aryl $\mathrm{C}), 139.2(\mathrm{C} \times 2$, aryl $\mathrm{CH}), 146.2$ and 154.6 ( $\mathrm{C} \times 4$, aryl C), 185.8 (C $\times 2, \mathrm{CHO}) . \mathrm{MS}(+\mathrm{El}, \mathrm{m} / \mathrm{z}, \%): 450(\mathrm{M}, 13), 419$ (11), 232 (33), 231 (49), 216 (45), 203 (100). Anal. calcd for $\mathrm{C}_{25} \mathrm{H}_{26} \mathrm{~N}_{2} \mathrm{O}_{6}: \mathrm{C}, 66.7 ; \mathrm{H}, 5.8 ; \mathrm{N}, 6.2$. Found: $\mathrm{C}, 66.7 ; \mathrm{H}, 5.5 ; \mathrm{N}, 6.0 \%$.

4,4'-Bis(1-acetyl-5,7-dimethoxyindolyl)methane (14). 1-Acetyl-5,7-dimethoxyindole 4 (0.10 g, $0.46 \mathrm{mmol})$, $37 \%$ aqueous formaldehyde solution $(0.03 \mathrm{~mL}, 0.40 \mathrm{mmol})$ and glacial acetic acid $(2 \mathrm{~mL})$ were combined and stirred at room temperature for $2 \mathrm{~d}$ under an inert gas atmosphere. The solution was diluted with water and 
the resulting turbid mixture was allowed to stand overnight before being filtered. The filtered material was washed with water and dried, affording 4,4'-di(1-acetyl-5,7-dimethoxyindolyl)methane 14 (23 mg, 22\%) as a grey powder. Radial chromatography using 1:39 methanol : dichloromethane gave a colourless oil which solidified to yield a white solid, $\mathrm{mp} 157-159{ }^{\circ} \mathrm{C}$. IR $\left(v_{\max } \mathrm{cm}^{-1}\right): 1692,1599,1371,1328,1268,1246,1221$, 1199, 1110, 1101. UV/Vis $\left(\lambda_{\max }, \mathrm{nm}, \varepsilon, \mathrm{cm}^{-1} \mathrm{M}^{-1}\right): 325(17,000) .{ }^{1} \mathrm{H} \mathrm{NMR}\left(300 \mathrm{MHz} \mathrm{CDCl}_{3}\right): \delta_{\mathrm{H}} 2.58(6 \mathrm{H}, \mathrm{s}$, COMe), 3.88 and $3.92(12 \mathrm{H}, \mathrm{s}, \mathrm{OMe}), 4.30\left(2 \mathrm{H}, \mathrm{s}, \mathrm{CH}_{2}\right), 6.53(2 \mathrm{H}, \mathrm{s}, \mathrm{H} 6), 6.66(2 \mathrm{H}, \mathrm{d}, J 3.8 \mathrm{~Hz}, \mathrm{H} 3), 7.47(2 \mathrm{H}, \mathrm{d}, J$ $3.8 \mathrm{~Hz}, \mathrm{H} 2) .{ }^{13} \mathrm{C} \mathrm{NMR}\left(75 \mathrm{MHz}, \mathrm{CDCl}_{3}\right): \delta_{\mathrm{C}} 22.4\left(\mathrm{CH}_{2}\right), 25.7(\mathrm{C} \times 2, \mathrm{COMe}), 56.0$ and $57.0(\mathrm{C} \times 4, \mathrm{OMe}), 94.7$ and 107.5 ( C x 4, aryl CH), 114.1 and 119.7 (C x 4, aryl C), 127.8 (C x 2, aryl CH), 134.7, 146.5 and 153.9 (C x 6, aryl C), 169.4 (C x 2, ㄷMe). MS (+EI, m/z, \%): 451 (M+1, 24), 450 (M, 100), 408 (45), 335 (38), 190 (47), 189 (52), 160 (86). HRMS ( $m / z)$ : Calcd for $\mathrm{C}_{25} \mathrm{H}_{26} \mathrm{~N}_{2} \mathrm{NaO}_{6}[\mathrm{M}+\mathrm{Na}]^{+}$, 473.1683. Found: 473.1656.

The filtrate was extracted with dichloromethane, and the organic phase was washed with water and saturated $\mathrm{NaHCO}_{3}$ solution, dried $\left(\mathrm{Na}_{2} \mathrm{SO}_{4}\right)$, and the solvent removed under reduced pressure affording 4,4'-di(1-acetyl5,7-dimethoxyindolyl)methane 14 (36 mg, 35\%) as an off-white powder. This product was confirmed by comparison with the other product obtained from this reaction using thin layer chromatography and ${ }^{1} \mathrm{H} N M R$ spectroscopy.

Dimethyl 4,4'-methylenebis[5,7-dimethoxy-1H-indole]-2,2'-dicarboxylate (15). Method A. A solution of methyl 4-hydroxymethyl-5,7-dimethoxyindole-2-carboxylate 16 (50 mg, 0.19 mmol) in glacial acetic acid (2.0 $\mathrm{mL}$ ) was stirred at room temperature for $5 \mathrm{~d}$. The resulting precipitate was filtered through a frit, washed twice with acetic acid $(1 \mathrm{~mL})$, then with water, and dried to give the title compound (33 $\mathrm{mg}, 73 \%$ ) as a white powder, $\mathrm{mp} 292-294{ }^{\circ} \mathrm{C}$. IR $\left(v_{\max }, \mathrm{cm}^{-1}\right)$ : 3321, 1690, 1590, 1536, 1437, 1351, 1330, 1253, 1211, 1094. UV/Vis $\left(\lambda_{\max }, \mathrm{nm}, \varepsilon, \mathrm{cm}^{-1} \mathrm{M}^{-1}\right): 241$ (54,700), $276(16,800, \mathrm{sh}), 297(53,200), 337(8,620) .{ }^{1} \mathrm{H} \mathrm{NMR}\left(300 \mathrm{MHz} \mathrm{CDCl}_{3}\right): \delta_{\mathrm{H}}$ 3.87, 3.88, 3.94 and (each $6 \mathrm{H}, \mathrm{s}, \mathrm{OMe}), 4.40\left(2 \mathrm{H}, \mathrm{s}\right.$, bridging $\left.\mathrm{CH}_{2}\right), 6.55(2 \mathrm{H}, \mathrm{s}, \mathrm{H} 6), 7.26(2 \mathrm{H}, \mathrm{d}, \mathrm{J} 2.3 \mathrm{~Hz}, \mathrm{H} 3)$, $8.79(2 \mathrm{H}, \mathrm{bs}, \mathrm{NH}) .{ }^{13} \mathrm{C} \mathrm{NMR}\left(75 \mathrm{MHz}, \mathrm{CDCl}_{3}\right): \delta_{\mathrm{c}} 22.6$ (bridging $\left.\mathrm{CH}_{2}\right), 51.6,55.4,58.2(\mathrm{OMe}), 62.2\left(\mathrm{CO}_{2} \mathrm{Me}\right), 95.5$ (C6), 108.7 (C3), 114.7, 124.2, 126.6, 128.8, 144.8, 151.2 (aryl C). MS (MALDI, m/z, \%): 482 (M, 36), 481 (M-1, 100). Anal. calcd for $\mathrm{C}_{25} \mathrm{H}_{26} \mathrm{~N}_{2} \mathrm{O}_{8}: \mathrm{C}, 62.2 ; \mathrm{H}, 5.4 ; \mathrm{N}, 5.81$. Found: $\mathrm{C}, 62.0 ; \mathrm{H}, 5.5 ; \mathrm{N}, 5.8 \%$.

Method B. A solution of methyl 5,7-dimethoxyindole-2-carboxylate 5 (50 $\mathrm{mg}, 0.21 \mathrm{mmol})$ and 4hydroxymethyl-5,7-dimethoxyindole-2-carboxylate 16 (56 $\mathrm{mg}, 0.21 \mathrm{mmol}$ ) in glacial acetic acid $(2.0 \mathrm{~mL})$ was stirred at room temperature for $75 \mathrm{~min}$. The resulting precipitate was filtered through a frit, washed successively with a little acetic acid, water, saturated $\mathrm{NaHCO}_{3}$ solution, water, and then dried to give the title compound (71 $\mathrm{mg}, 70 \%$ ) as a white powder.

Method C. A mixture of methyl 5,7-dimethoxyindole-2-carboxylate 5 (0.25 g, $1.06 \mathrm{mmol})$ and formaldehyde $(0.2 \mathrm{~mL})$ in anhydrous methanol $(7 \mathrm{~mL})$ was stirred at room temperature with concentrated $\mathrm{HCl}(1 \mathrm{drop})$ for 19 h. The resulting precipitate was filtered through a frit, washed with water, and dried to give the title compound (70 $\mathrm{mg}, 27 \%$ ) as a white powder.

Method D. A mixture of methyl 5,7-dimethoxyindole-2-carboxylate 5 (0.25 g, $1.06 \mathrm{mmol})$ and $40 \%$ aqueous formaldehyde (10 drops) in glacial acetic acid $(4.0 \mathrm{~mL})$ was stirred at room temperature for $3 \mathrm{~d}$. The resulting precipitate was filtered through a frit, washed with water, and dried to give the title compound (0.22 g, 85\%) as a white powder.

Methyl 4-hydroxymethyl-5,7-dimethoxyindole-2-carboxylate (16). Sodium borohydride (1.44 g, $38 \mathrm{mmol})$ was added portionwise to a stirred suspension of methyl 4-formyl-5,7-dimethoxyindole-2-carboxylate 11 (1.00 $\mathrm{g}, 3.80 \mathrm{mmol})$ in methanol $(100 \mathrm{~mL})$ and stirring was continued at room temperature for $5.5 \mathrm{~h}$. The mixture was then diluted with water and extracted with ethyl acetate. The organic extract was dried $\left(\mathrm{MgSO}_{4}\right)$ and the solvent evaporated in vacuo to give the title compound $(0.78 \mathrm{~g}, 77 \%)$ as a white powder, $\mathrm{mp} 148-151{ }^{\circ} \mathrm{C}$. IR $\left(v_{\max } \mathrm{cm}^{-1}\right)$ : 3477, 3177, 1717, 1698, 1594, 1538, 1455, 1432, 1327, 1260, 1217, 1199, 1175, 1148, 1122, 1094, 
990. UV/Vis $\left(\lambda \max , \mathrm{nm}, \varepsilon, \mathrm{cm}^{-1} \mathrm{M}^{-1}\right): 242(29,300), 295(19,000), 327(4,640) ; \delta_{\mathrm{H}}\left(300 \mathrm{MHz}, \mathrm{CDCl}_{3}\right) 2.16(1 \mathrm{H}, \mathrm{bs}$, $\mathrm{CH}_{2} \mathrm{O} \underline{\mathrm{H}}$ ), 3.97, 3.93, 3.91 (each 3H, s, OMe), $4.91\left(2 \mathrm{H}, \mathrm{d}, J 3.0 \mathrm{~Hz}, \mathrm{CH}_{2} \mathrm{OH}\right), 6.52(1 \mathrm{H}, \mathrm{s}, \mathrm{H} 6), 7.25(1 \mathrm{H}, \mathrm{d}, J 2.3 \mathrm{~Hz}$, $\mathrm{H} 3), 9.01(1 \mathrm{H}, \mathrm{bs}, \mathrm{NH}) .{ }^{13} \mathrm{C} \mathrm{NMR}\left(75 \mathrm{MHz} \mathrm{CDCl}_{3}\right): \delta_{\mathrm{C}} 51.9,55.5,57.8$ and $(\mathrm{OMe}) ; 57.8\left(\underline{\mathrm{C}} \mathrm{H}_{2} \mathrm{OH}\right), 94.0$ (C6), 106.8 (C3), 112.7, 123.7, 127.6, 128.0, 146.4, 152.5 (aryl C), 162.0 ( $\mathrm{CO}_{2} \mathrm{Me}$ ). MS (+El, m/z, \%): 266 (10), 265 (M, 100$)$, 248 (23), 234 (17), 233 (80), 232 (37), 216 (76), 204 (25). Anal. calcd for $\mathrm{C}_{13} \mathrm{H}_{15} \mathrm{NO}_{5} .0 .33 \mathrm{H} \mathrm{O}_{2} \mathrm{C}, 57.6 ; \mathrm{H}, 5.8 ; \mathrm{N}$, 5.2. Found: $C, 57.4 ; H, 5.7 ; N, 5.0 \%$.

Methyl 1-(tert-butyloxycarbonyl)-4-hydroxymethyl-5,7-dimethoxyindole-2-carboxylate (17). Sodium borohydride $(0.12 \mathrm{~g}, 3.2 \mathrm{mmol})$ was added portionwise, over $15 \mathrm{~min}$, to a stirred suspension of methyl 1-(tertbutyloxycarbonyl)-4-formyl-5,7-dimethoxyindole-2-carboxylate 12 (0.30 g, $0.83 \mathrm{mmol}$ ) in methanol (20 mL). After stirring for $15 \mathrm{~min}$ further at room temperature, the solvent was evaporated in vacuo and the remaining residue suspended in $1 \mathrm{M} \mathrm{NaOH}$ and extracted with ethyl acetate. The organic extract was washed twice with $1 \mathrm{M} \mathrm{NaOH}$, then twice with brine, dried $\left(\mathrm{MgSO}_{4}\right)$, and the solvent evaporated in vacuo to give the title compound $(0.273 \mathrm{~g}, 90 \%)$ as a colourless crystalline solid, $\mathrm{mp} 138-141{ }^{\circ} \mathrm{C}$. IR $\left(\mathrm{v}_{\max }, \mathrm{cm}^{-1}\right): 3559,1765,1704$, 1592, 1542, 1437, 1253, 1222, 1158, 1144, 1089, 999. UV/Vis $\left(\lambda_{\max }, \mathrm{nm}, \varepsilon, \mathrm{cm}^{-1} \mathrm{M}^{-1}\right): 241(27,800), 294$ (17,600), 334 (4,580). ${ }^{1} \mathrm{H}$ NMR $\left(300 \mathrm{MHz} \mathrm{CDCl}_{3}\right): \delta_{\mathrm{H}} 1.64\left(9 \mathrm{H}, \mathrm{s}, \mathrm{CMe}_{3}\right), 2.05\left(1 \mathrm{H}, \mathrm{bs}, \mathrm{CH}_{2} \mathrm{OH}\right), 3.93,3.90,3.89$ (each $3 \mathrm{H}, \mathrm{s}, \mathrm{OMe}), 4.87\left(2 \mathrm{H}, \mathrm{s}, \mathrm{CH}_{2} \mathrm{OH}\right), 6.55(1 \mathrm{H}, \mathrm{s}, \mathrm{H} 6), 7.28(1 \mathrm{H}, \mathrm{s}, \mathrm{H} 3) .{ }^{13} \mathrm{C} \mathrm{NMR}\left(75 \mathrm{MHz}, \mathrm{CDCl}_{3}\right): \delta_{\mathrm{C}} 27.3$ $\left(\mathrm{CMe}_{3}\right), 51.9,55.6,57.5$ (OMe), $57.5\left(\mathrm{CH}_{2} \mathrm{OH}\right), 84.6$ (CMe$\left.)_{3}\right), 95.3$ (C6), 109.1 (C3), $161.0\left(\mathrm{CO}_{2} \mathrm{Me}\right), 112.8,123.2$, 127.6, 128.3, 146.9, 150.5, 153.0 (aryl C). MS (+EI, m/z, \%): 365 (M, 6), 292 (10), 265 (77), 248 (20), 234 (13), 233 (100), 216 (36), 204 (22), 149 (22). Anal. calcd for $\mathrm{C}_{18} \mathrm{H}_{23} \mathrm{NO}_{7}$ : C, 59.2; H, 6.3; N, 3.8. Found: C, 59.0; H, 6.4; N, 3.8\%.

\section{Dimethyl 4,4'-methylenebis[1-(tert-butyloxycarbonyl)-5,7-dimethoxy-1H-indole]-2,2'-dicarboxylate (18).}

Method A. A solution of bromine $(27 \mathrm{mg}, 0.17 \mathrm{mmol})$ in carbon tetrachloride $(1.5 \mathrm{~mL})$ was added dropwise to a stirred suspension of triphenylphosphine $(40 \mathrm{mg}, 0.15 \mathrm{mmol})$ in carbon tetrachloride $(1.0 \mathrm{~mL})$. The resulting yellow suspension was stirred further for $25 \mathrm{~min}$, under nitrogen, at room temperature before triethylamine $(17 \mathrm{mg}, 0.17 \mathrm{mmol})$ in carbon tetrachloride $(1.0 \mathrm{~mL})$ was added and stirring continued for $20 \mathrm{~min}$. A solution of methyl 1-(tert-butyloxycarbonyl)-4-hydroxymethyl-5,7-dimethoxyindole-2-carboxylate 17 (0.56 mg, 0.15 $\mathrm{mmol})$ in carbon tetrachloride $(4 \mathrm{~mL})$ was then added dropwise and stirring continued for $2 \mathrm{~d}$. The solvent was then evaporated in vacuo and the remaining residue purified via gravity column chromatography (2\% $\mathrm{MeOH} / \mathrm{CH}_{2} \mathrm{Cl}_{2}$ ) to give the title compound (39 mg, 75\%) as a colourless crystalline solid, $\mathrm{mp} 179-180{ }^{\circ} \mathrm{C}$. IR $\left(v_{\text {max }}, \mathrm{cm}^{-1}\right): 1771,1720,1590,1396,1371,1258,1234,1157,1083$. UV/Vis $\left(\lambda_{\max }, \mathrm{nm}, \varepsilon, \mathrm{cm}^{-1} \mathrm{M}^{-1}\right): 241$ (54,000), 294 (33,800), $344(10,000) .{ }^{1} \mathrm{H}$ NMR $\left(300 \mathrm{MHz}, \mathrm{CDCl}_{3}\right): \delta_{\mathrm{H}} 1.60\left(18 \mathrm{H}, \mathrm{s}, \mathrm{CMe}_{3}\right), 3.86(12 \mathrm{H}, \mathrm{s}, \mathrm{OMe})$, $3.89(6 \mathrm{H}, \mathrm{s}, \mathrm{OMe}), 4.32\left(2 \mathrm{H}, \mathrm{s}\right.$, bridging $\left.\mathrm{CH}_{2}\right), 6.56(2 \mathrm{H}, \mathrm{s}, \mathrm{H} 6), 7.37(2 \mathrm{H}, \mathrm{s}, \mathrm{H} 3) .{ }^{13} \mathrm{C} \mathrm{NMR}\left(75 \mathrm{MHz} \mathrm{CDCl}_{3}\right): \delta_{\mathrm{C}}$ 22.3 (bridging $\mathrm{CH} 2$ ), $27.3\left(\mathrm{CMe}_{3}\right), 51.7,55.5,57.5$, (OMe), 84.2 ( $\left.\mathrm{CMe}_{3}\right), 96.2$ (C6), 111.1 (C3), 114.2, 123.5, 127.3, 128.3, 145.4, 150.8, 151.7 (CO and aryl C), 161.2 ( $\left.\mathrm{CO}_{2} \mathrm{Me}\right)$. MS (MALDI, $\left.m / z, \%\right): 682$ (M, 42$), 582$ (18), 482 (100), 248 (69). Anal. calcd for $\mathrm{C}_{35} \mathrm{H}_{42} \mathrm{~N}_{2} \mathrm{O}_{12}$ : C, 61.6; H, 6.2; N, 4.1. Found: C, 61.5; $\mathrm{H}, 6.3 ; \mathrm{N}, 4.0 \%$. Method B. A suspension of dimethyl 4,4'-methylenebis[5,7-dimethoxy-1H-indole]-2,2'-dicarboxylate 15 (50 $\mathrm{mg}, 0.10 \mathrm{mmol})$ and di-(t-butyl)carbonate $(125 \mathrm{mg}, 0.57 \mathrm{mmol})$ in acetonitrile $(3.0 \mathrm{~mL})$ was stirred at room temperature, under nitrogen, with a catalytic quantity of 4-dimethylaminopyridine for $23 \mathrm{~h}$. The solvent was then evaporated in vacuo and the remaining residue purified via suction column chromatography (1\% $\mathrm{MeOH} / \mathrm{CH}_{2} \mathrm{Cl}_{2}$ ) to give compound 18 (67 mg, 95\%) as a white solid.

Dimethyl [1,3,4,5-tetrahydro-6,8-dimethoxybenz[c,d]indole]-4-spiro-4'-[1,4,7-trihydro-5'-methoxyindol-7one]-2,2'-dicarboxylate (19). Methyl 4-hydroxymethyl-5,7-dimethoxyindole-2-carboxylate 16 (77 mg, 0.30 $\mathrm{mmol}$ ) in methanol $(15 \mathrm{~mL})$ was stirred at room temperature with concentrated $\mathrm{HCl}(1 \mathrm{drop})$ for $3 \mathrm{~h}$. The resulting precipitate was filtered through a frit, washed with water, and dried to give the title compound (66 
$\mathrm{mg}, 91 \%)$ as a pale yellow powder, $\mathrm{mp} 291-292{ }^{\circ} \mathrm{C}$. IR $\left(v_{\max }, \mathrm{cm}^{-1}\right): 3324,1727,1696,1641,1464,1334,1278$, 1254, 1229. UV/Vis $\left(\lambda_{\max }, \mathrm{nm}, \varepsilon, \mathrm{cm}^{-1} \mathrm{M}^{-1}\right): 244$ (41,000), $287(24,000), 299(34,500), 312(21,700), 347(7,300)$. ${ }^{1} \mathrm{H} N M R\left(300 \mathrm{MHz}, \mathrm{CDCl}_{3}\right): \delta_{\mathrm{H}} 2.88\left(1 \mathrm{H}, \mathrm{d}, J 15.8 \mathrm{~Hz}, \mathrm{CH}_{2}\right), 3.08\left(1 \mathrm{H}, \mathrm{d}, J 16.6 \mathrm{~Hz}, \mathrm{CH}_{2}\right), 3.44(1 \mathrm{H}, \mathrm{d}, J 15.8 \mathrm{~Hz}$, $\left.\mathrm{CH}_{2}\right), 3.61\left(1 \mathrm{H}, \mathrm{d}, J 16.6 \mathrm{~Hz}, \mathrm{CH}_{2}\right), 3.71,3.78,3.85,3.89,4.01$ (each 3H, s, OMe), $5.37\left(1 \mathrm{H}, \mathrm{d}, J 2.3 \mathrm{~Hz}, \mathrm{H} 3^{\prime}\right), 5.81$ $\left(1 \mathrm{H}, \mathrm{s}, \mathrm{H6} 6^{\prime}\right), 6.55(1 \mathrm{H}, \mathrm{s}, \mathrm{H6}), 8.74,9.58(2 \mathrm{H}, \mathrm{bs}, \mathrm{NH}) .{ }^{13} \mathrm{C} \mathrm{NMR}\left(75 \mathrm{MHz}, \mathrm{CDCl}_{3}\right): \delta_{\mathrm{C}} 32.5,33.3\left(\mathrm{CH}_{2}\right), 44.2$ (alkyl C), 52.1, 56.1, 57.0, 58.4 (OMe), 97.1 (C6), 102.8 (C6'), 112.9 (C3'), 108.2, 118.9, 121.3, 123.6, 126.0, 128.2, 128.3, 136.3, 145.6, 151.1 (aryl C), 161.4, 162.9 ( $\left.\mathrm{CO}_{2} \mathrm{Me}\right), 178.3$ and 181.0 (C5 and quinone CO). MS (MALDI, $m / z, \%): 503$ (M+Na, 10), 480 (M, 52), 479 (100); MS (+El, m/z, \%): 481 (23), 480 (M, 100), 449 (27), 448 (37), 421 (41), 417 (16), 416 (13), 389 (43), 345 (13), 167 (22), 149 (50). HRMS (m/z): Calcd for $\mathrm{C}_{25} \mathrm{H}_{24} \mathrm{~N}_{2} \mathrm{O}_{8}[\mathrm{M}]^{+}$, 480.1533. Found: 480.1528. Anal. calcd for $\mathrm{C}_{25} \mathrm{H}_{24} \mathrm{~N}_{2} \mathrm{O}_{8} .0 .5 \mathrm{H}_{2} \mathrm{O}: \mathrm{C}, 61.3 ; \mathrm{H}, 5.1 ; \mathrm{N}$, 5.7. Found: $\mathrm{C}, 61.2 ; \mathrm{H}, 4.9$; N, $5.8 \%$.

Acid catalyzed reaction of methyl 1-(tert-butyloxycarbonyl)-4-hydroxymethyl-5,7-dimethoxyindole-2carboxylate (17). Methyl 1-(tert-butyloxycarbonyl)-4-hydroxymethyl-5,7-dimethoxyindole-2-carboxylate 17 (50 mg, $0.14 \mathrm{mmol}$ ) in methanol $(1.5 \mathrm{~mL}$ ) was stirred at room temperature with concentrated $\mathrm{HCl}(1 \mathrm{drop})$ for $40 \mathrm{~min}$. The resulting precipitate was filtered through a frit, washed sequentially with a little methanol and water, and dried to give the diindolylmethane $18(8 \mathrm{mg}, 17 \%)$ as a white powder. The reaction filtrate and methanolic washings were combined and stirred for a further $4 \mathrm{~d}$. The solvent was then evaporated in vacuo and the remaining residue purified via gravity column chromatography $\left(1 \% \mathrm{MeOH} / \mathrm{CH}_{2} \mathrm{Cl}_{2}\right)$ to give the three following products:

Dimethyl 4,4'-methylenebis[1-(tert-butyloxycarbonyl)-5,7-dimethoxy-1H-indole]-2,2'-dicarboxylate (18) (6 $\mathrm{mg}, 13 \%)$ was obtained as a white powder.

Dimethyl [1-(tert-butyloxycarbonyl)-1,3,4,5-tetrahydro-6,8-dimethoxybenz[c,d]indole]-4-spiro-4'-[1-(tertbutyloxycarbonyl)-1,4,7-trihydro-5'-methoxyindol-7-one]-2,2'-dicarboxylate (20). (21 mg, 45\%) was obtained as a white powder, $\mathrm{mp} 153-15{ }^{\circ} \mathrm{C}$. IR $\left(v_{\max }, \mathrm{cm}^{-1}\right): 1779,1720,1594,1456,1371,1257,1220,1157$. UV/Vis $\left(\lambda_{\max }, \mathrm{nm}, \varepsilon, \mathrm{cm}^{-1} \mathrm{M}^{-1}\right): 246(27,000), 297(17,000), 340(13,000) .{ }^{1} \mathrm{H} \mathrm{NMR}\left(300 \mathrm{MHz}, \mathrm{CDCl}_{3}\right): \delta_{\mathrm{H}} 1.61$ and 1.64 (each $\left.9 \mathrm{H}, \mathrm{s}, \mathrm{CMe}_{3}\right), 2.93\left(1 \mathrm{H}, \mathrm{d}, J 15.8 \mathrm{~Hz}, \mathrm{CH}_{2}\right), 3.07\left(1 \mathrm{H}, \mathrm{d}, J 17.0 \mathrm{~Hz}, \mathrm{CH}_{2}\right), 3.16\left(1 \mathrm{H}, \mathrm{d}, J 16.6 \mathrm{~Hz}, \mathrm{CH}_{2}\right), 3.42$ $\left(1 \mathrm{H}, \mathrm{d}, J 17.0 \mathrm{~Hz}, \mathrm{CH}_{2}\right), 3.68,3.78,3.82,3.90,3.96$ (each 3H, s, OMe), $5.52\left(1 \mathrm{H}, \mathrm{s}, \mathrm{H} 3^{\prime}\right), 5.64\left(1 \mathrm{H}, \mathrm{s}, \mathrm{H} 6^{\prime}\right), 6.58$ $(1 \mathrm{H}, \mathrm{s}, \mathrm{H} 6) .{ }^{13} \mathrm{C} \mathrm{NMR}\left(75 \mathrm{MHz}, \mathrm{CDCl}_{3}\right): \delta_{\mathrm{c}} 27.7$ and $27.9\left(\mathrm{CMe}_{3}\right), 33.3$ and $33.6\left(\mathrm{CH}_{2}\right), 43.4$ (alkyl C), 52.1, 52.2, 56.3, 56.5, 57.9 (OMe), 84.6 and $86.4\left(\mathrm{CMe}_{3}\right), 98.5$ and 99.6 (C6' and C6), 114.5 (C3'), 109.1, 122.6, 125.5, 128.0, 133.3, 146.4, 149.8, 151.3, 152.3, 160.6 (aryl C), 162.0 and 163.1 ( $\left.\underline{\mathrm{CO}}_{2} \mathrm{Me}\right), 177.2$ and 179.6 (C5' and quinone CO). MS (MALDI, $m / z, \%): 482$ (76), 450 (100), 422 (97).

Methyl 1-(tert-butyloxycarbonyl)-5,7-dimethoxy-4-methoxymethylindole-2-carboxylate (21). (6 mg, 12\%) was obtained as a pale yellow powder. ${ }^{1} \mathrm{H} \mathrm{NMR}\left(300 \mathrm{MHz}, \mathrm{CDCl}_{3}\right): \delta_{\mathrm{H}} 1.64\left(9 \mathrm{H}, \mathrm{s}, \mathrm{CMe}_{3}\right), 3.35\left(3 \mathrm{H}, \mathrm{s}, \mathrm{CH}_{2} \mathrm{OMe}\right)$, 3.88, 3.90, $3.93(9 \mathrm{H}, \mathrm{s}, \mathrm{OMe}), 4.70\left(2 \mathrm{H}, \mathrm{s}, \mathrm{CH}_{2} \mathrm{OMe}\right), 6.55(1 \mathrm{H}, \mathrm{s}, \mathrm{H} 6), 7.30(1 \mathrm{H}, \mathrm{s}, \mathrm{H} 3) .{ }^{13} \mathrm{C} \mathrm{NMR}(75 \mathrm{MHz}$, $\left.\mathrm{CDCl}_{3}\right): \delta_{\mathrm{c}} 27.3$ ( $\left.\mathrm{CMe}_{3}\right), 51.9,55.6,57.6,58.0$ (OMe), 65.7 ( $\left.\mathrm{CH}_{2} \mathrm{OMe}\right), 84.5\left(\mathrm{CMe}_{3}\right), 95.8$ (C6), 109.9 (C3), 161.1 $\left(\mathrm{CO}_{2} \mathrm{Me}\right), 110.4,123.4,128.1,128.5,147.1,150.6,153.5$ (CO and aryl C).

3,3'-Bis(5,7-dimethoxy-1-methylindolyl)phenylmethane (23). 5,7-Dimethoxy-1-methylindole 1 (0.10 g, 0.53 $\mathrm{mmol})$, benzaldehyde $(0.05 \mathrm{~mL}, 0.53 \mathrm{mmol})$ and glacial acetic acid $(2 \mathrm{~mL})$ were combined and stirred at room temperature for $3 \mathrm{~h}$. The resulting precipitate was filtered, washed with water and dried to give 3,3'-bis-(5,7dimethoxy-1-methylindolyl)phenylmethane $23(94 \mathrm{mg}, 76 \%)$ as a white powder, $\mathrm{mp} 195{ }^{\circ} \mathrm{C} . \mathrm{IR}\left(\mathrm{v}_{\max }, \mathrm{cm}^{-1}\right)$ : $1582,1497,1458,1414,1279,1205,1147,1117,1047,809$. UV/Vis $\left(\lambda_{\max }, \mathrm{nm}, \varepsilon, \mathrm{cm}^{-1} \mathrm{M}^{-1}\right): 213(49,000), 229$ (54,000), 266 (12,000), 273 (11,000), 299 (11,000), $307(10,000) .{ }^{1} \mathrm{H} \mathrm{NMR}\left(300 \mathrm{MHz}^{\mathrm{CDCl}}\right)_{3}: \delta_{\mathrm{H}} 3.66(6 \mathrm{H}, \mathrm{s}$, NMe), $3.88(12 \mathrm{H}, \mathrm{s}, \mathrm{OMe}), 5.64(1 \mathrm{H}, \mathrm{br} \mathrm{s}, \mathrm{CH}), 6.28(2 \mathrm{H}, \mathrm{d}, J 2.0 \mathrm{~Hz}, \mathrm{H} 6), 6.33(2 \mathrm{H}, \mathrm{d}, J 2.0 \mathrm{~Hz}, \mathrm{H} 4), 6.37(2 \mathrm{H}, \mathrm{s}$, 
$\mathrm{H} 2), 7.23\left(5 \mathrm{H}, \mathrm{m}\right.$, aryl H). $\left.{ }^{13} \mathrm{C} \mathrm{NMR}\left(75 \mathrm{MHz}^{\mathrm{CDCl}}\right)_{3}\right): \delta_{\mathrm{c}} 36.1$ (2C, NMe), $40.1(\mathrm{CH}), 55.3$ and 55.7 (4C, OMe), 93.3 and 94.2 (4C, aryl $\mathrm{CH}$ ), 117.8 and 122.5 (4C, aryl C), 125.8 (phenyl $\mathrm{CH}$ ), 128.1 and 128.7 (4C, phenyl $\mathrm{CH}$ ), 129.1 (2C, aryl CH), 129.6 (2C, aryl CH), 144.5 (phenyl C), 147.9 and 153.9 (4C, aryl C). MS (+El, m/z, \%): 471 (M, 28), 470 (100), 455 (32), 394 (17), 393 (72), 235 (28), 177 (40). HRMS (m/z): Calcd for $\mathrm{C}_{29} \mathrm{H}_{30} \mathrm{~N}_{2} \mathrm{NaO}_{4}$ $[\mathrm{M}+\mathrm{Na}]^{+}$, 493.2098. Found: 493.2108. Anal. calcd for $\mathrm{C}_{29} \mathrm{H}_{30} \mathrm{~N}_{2} \mathrm{O}_{4}: \mathrm{C}, 74.0 ; \mathrm{H}, 6.4 ; \mathrm{N}, 6.0$. Found: $\mathrm{C}, 73.7 ; \mathrm{H}, 6.4$; N, $5.6 \%$.

3,3'-Bis(5,7-dimethoxy-1-methylindolyl)-4-chlorophenylmethane (24). 5,7-Dimethoxy-1-methylindole 1 (0.20 g, $1.05 \mathrm{mmol})$, 4-chlorobenzaldehyde $(0.15 \mathrm{~g}, 1.05 \mathrm{mmol})$ and glacial acetic acid $(4 \mathrm{~mL})$ were combined and stirred at room temperature for $3 \mathrm{~h}$. The resulting precipitate was filtered, washed with water and dried to give 3,3'-di(5,7-dimethoxy-1-methylindolyl)-4-chlorophenylmethane 24 (0.22 g, 83\%) as an off-white powder. Recrystallisation from dichloromethane and petroleum ether afforded a white powder, $\mathrm{mp} 194{ }^{\circ} \mathrm{C}$. IR $\left(v_{\max }\right.$ $\left.\mathrm{cm}^{-1}\right): 1582,1500,1454,1413,1280,1203,1148,1118,1049,804$. UV/Vis $\left(\lambda_{\max }, \mathrm{nm}, \varepsilon, \mathrm{cm}^{-1} \mathrm{M}^{-1}\right): 262(12,000)$, 299 (9,300), 308 (8,300). ${ }^{1} \mathrm{H}$ NMR $\left(300 \mathrm{MHz}_{\mathrm{CDCl}}\right): \delta_{\mathrm{H}} 3.68,3.88$ and $3.88(18 \mathrm{H}, \mathrm{s}, \mathrm{OMe}$ and NMe), $5.61(1 \mathrm{H}$, br s, CH), $6.29\left(4 \mathrm{H}, \mathrm{m}\right.$, aryl H), $6.34(2 \mathrm{H}, \mathrm{d}, J 0.85 \mathrm{~Hz}, \mathrm{H6}), 7.23(2 \mathrm{H}, \mathrm{d}, J 0.85 \mathrm{~Hz}, \mathrm{H} 4), 7.23(2 \mathrm{H}, \mathrm{s}, \mathrm{H} 2) .{ }^{13} \mathrm{C} \mathrm{NMR}$ $\left(75 \mathrm{MHz}, \mathrm{CDCl}_{3}\right): \delta_{\mathrm{C}} 36.1$ (2C, NMe), $39.5(\mathrm{CH}), 55.3$ and $55.8(4 \mathrm{C}, \mathrm{OMe}), 93.1$ and $94.3(4 \mathrm{C}$, aryl $\mathrm{CH}), 117.3$ and 122.5 (4C, aryl C), 128.2 (2C, phenyl CH), 128.9 (2C, aryl C), 129.6 (2C, aryl CH), 130.0 (2C, phenyl CH), 131.5 and $143.1(\operatorname{aryl~C}), 148.0$ and $154.0\left(4 \mathrm{C}\right.$, aryl C). $\mathrm{MS}(+\mathrm{El}, \mathrm{m} / \mathrm{z}, \%): 507\left(\mathrm{M},{ }^{37} \mathrm{Cl}\right.$ isotope, 11$), 506\left({ }^{37} \mathrm{Cl}\right.$ isotope, 36), 505 (M, ${ }^{35} \mathrm{Cl}$ isotope, 35), $504\left({ }^{35} \mathrm{Cl}\right.$ isotope, 100), 503 (15), 489 (32), 393 (68). HRMS (m/z): Calcd for $\mathrm{C}_{29} \mathrm{H}_{29} \mathrm{ClN}_{2} \mathrm{NaO}_{4}[\mathrm{M}+\mathrm{Na}]^{+}$, 527.1708. Found: 527.1734. Anal. calcd for $\mathrm{C}_{29} \mathrm{H}_{29} \mathrm{ClN}_{2} \mathrm{O}_{4}: \mathrm{C}, 69.0 ; \mathrm{H}, 5.8 ; \mathrm{N}, 5.5$. Found: $\mathrm{C}, 68.7 ; \mathrm{H}, 5.7 ; \mathrm{N}, 5.15 \%$.

3,3'-Bis(5,7-dimethoxy-1-methylindolyl)-4-hydroxyphenylmethane (25). 5,7-Dimethoxy-1-methylindole 1 $(0.25 \mathrm{~g}, 1.31 \mathrm{mmol}), 4$-hydroxybenzaldehyde $(0.12 \mathrm{~g}, 0.99 \mathrm{mmol})$ and glacial acetic acid $(5 \mathrm{~mL})$ were combined and stirred at room temperature for $5 \mathrm{~h}$. The resulting precipitate was filtered, washed with water and dried to give 3,3'-di(5,7-dimethoxy-1-methylindolyl)-4-hydroxyphenylmethane $25(0.29 \mathrm{~g}, 91 \%)$ as a white powder, $\mathrm{mp} 227^{\circ} \mathrm{C}$. IR $\left(v_{\max }, \mathrm{cm}^{-1}\right)$ : 3396, 1582, 1511, 1498, 1455, 1280, 1205, 1148, 1117, $1044 \mathrm{~cm}$. UV/Vis $\left(\lambda_{\max }, \mathrm{nm}\right.$, $\left.\varepsilon, \mathrm{cm}^{-1} \mathrm{M}^{-1}\right): 217$ (43,000), 230 (48,000), 267 (12,000), 272 (12,000), 299 (11,000), 307 (9,700). ${ }^{1} \mathrm{H}$ NMR (300 $\left.\mathrm{MHz}, \mathrm{CDCl}_{3}\right): \delta_{\mathrm{H}} 3.67,3.87$ and $3.88(18 \mathrm{H}, \mathrm{s}, \mathrm{OMe}$ and $\mathrm{NMe}), 5.58(1 \mathrm{H}, \mathrm{br} \mathrm{s}, \mathrm{CH}), 6.28(2 \mathrm{H}, \mathrm{d}, J 2.0 \mathrm{~Hz}, \mathrm{H} 4), 6.33$ $(2 \mathrm{H}, \mathrm{d}, J 2.0 \mathrm{~Hz}, \mathrm{H} 6), 6.35(2 \mathrm{H}, \mathrm{s}, \mathrm{H} 2), 6.72$ and $7.17(4 \mathrm{H}, \mathrm{m}$, aryl H$) .{ }^{13} \mathrm{C} \mathrm{NMR}\left(75 \mathrm{MHz}, \mathrm{CDCl}_{3}\right): \delta_{\mathrm{C}} 36.1(2 \mathrm{C}$, $\mathrm{NMe}), 39.2(\mathrm{CH}), 55.3$ and 55.8 (4C, OMe), 93.4, 94.2 and 114.9 (6C, aryl $\mathrm{CH}), 118.1,122.5$ and 129.1 (6C, aryl C), 129.6 and 129.7 (4C, aryl CH), 136.9 (aryl C), 147.9 (2C, aryl C), 153.6 (aryl C), 153.9 (2C, aryl C). MS (+EI, m/z, \%): 487 (M, 29), 486 (100), 485 (23), 471 (44), 393 (49), 295 (16), 243 (19), 191 (28). HRMS (m/z): Calcd for $\mathrm{C}_{29} \mathrm{H}_{30} \mathrm{~N}_{2} \mathrm{NaO}_{5}[\mathrm{M}+\mathrm{Na}]^{+}$, 509.2047. Found: 509.2020.

3,3'-Bis(5,7-dimethoxy-1-methylindolyl)-4-nitrophenylmethane (26). 5,7-Dimethoxy-1-methylindole 1 (0.20 $\mathrm{g}, 1.05 \mathrm{mmol}), 4-n i t r o b e n z a l d e h y d e(0.16 \mathrm{~g}, 1.05 \mathrm{mmol})$ and glacial acetic acid $(4 \mathrm{~mL})$ were combined and stirred at room temperature for $5 \mathrm{~h}$. The resulting precipitate was filtered, washed with water and dried to give 3,3'-di(5,7-dimethoxy-1-methylindolyl)-4-nitrophenylmethane $26(0.18 \mathrm{~g}, 66 \%)$ as a yellow powder. Recrystallisation from dichloromethane and petroleum ether afforded a fibrous yellow solid, $\mathrm{mp} 221-222{ }^{\circ} \mathrm{C}$. IR $\left(v_{\max }, \mathrm{cm}^{-1}\right): 1583,1514,1491,1455,1341,1280,1208,1148,1118,991$. UV/Vis $\left(\lambda_{\max }, \mathrm{nm}, \varepsilon, \mathrm{cm}^{-1} \mathrm{M}^{-1}\right): 216$ (49,000), 228 (54,000), 272 (22,000), 295 (17,000), 306 (14,000). ${ }^{1} \mathrm{H} N M R\left(300 \mathrm{MHz}, \mathrm{CDCl}_{3}\right): \delta_{\mathrm{H}} 3.67(6 \mathrm{H}, \mathrm{s}$, NMe), $3.89(12 \mathrm{H}, \mathrm{s}, \mathrm{OMe}), 5.74(1 \mathrm{H}, \mathrm{br} \mathrm{s}, \mathrm{CH}), 6.26$ and $6.30(4 \mathrm{H}, \mathrm{d}, J 2.0 \mathrm{~Hz}, \mathrm{H} 6$ and H4), $6.35(2 \mathrm{H}, \mathrm{s}, \mathrm{H} 2), 7.46$ and $8.13\left(4 \mathrm{H}, \mathrm{m}\right.$, aryl H). ${ }^{13} \mathrm{C} \mathrm{NMR}\left(75 \mathrm{MHz}^{\mathrm{CDCl}} 3\right): \delta_{\mathrm{C}} 36.2(2 \mathrm{C}, \mathrm{NMe}), 40.0(\mathrm{CH}), 55.4$ and 55.7 (4C, OMe), 92.7 and 94.4 (4C, aryl CH), 116.1 and 122.5 (4C, aryl C), 123.5 (2C, aryl CH), $128.6(2 \mathrm{C}$, aryl C), 129.4 and 129.6 (4C, aryl CH), $146.3(\operatorname{aryl} C), 148.1(2 \mathrm{C}$, aryl C), $152.5(\operatorname{aryl} C), 154.2$ (2C, aryl C). MS (+El, m/z, \%): 516 (M, 31), 
515 (100), 500 (22), 394 (18), 393 (65), 258 (15), 204 (17). Anal. calcd for $\mathrm{C}_{29} \mathrm{H}_{29} \mathrm{~N}_{3} \mathrm{O}_{6}:$ C, 67.6; H, 5.7; N, 8.2. Found: $C, 67.8 ; \mathrm{H}, 5.7 ; \mathrm{N}, 7.9 \%$.

3,3'-Bis(5,7-dimethoxy-1-methylindolyl)phenylmethane-4,4'-dicarbaldehyde (27). Phosphoryl chloride (0.10 $\mathrm{mL}, 1.07 \mathrm{mmol}$ ) was added to an ice-cold solution of 3,3'-di(5,7-dimethoxy-1-methylindolyl)phenylmethane 23 $(0.10 \mathrm{~g}, 0.21 \mathrm{mmol})$ in dry $\mathrm{N}, \mathrm{N}$-dimethylformamide $(3 \mathrm{~mL})$ with stirring and cooling in ice, and the reaction mixture was heated with stirring at $70{ }^{\circ} \mathrm{C}$ for $15 \mathrm{~min}$. After cooling, the reaction mixture was poured over ice and basified using $10 \%$ aqueous sodium hydroxide solution. After standing overnight, the precipitate was filtered, washed with water and dried to give 3,3'-di(5,7-dimethoxy-1-methylindolyl)phenylmethane-4,4'dicarbaldehyde 27 (93 mg, 83\%) as a brown powder. Recrystallisation from dichloromethane and petroleum ether afforded dark cream needles, $\mathrm{mp} 158-159{ }^{\circ} \mathrm{C}$. IR $\left(v_{\max }, \mathrm{cm}^{-1}\right): 1656,1650,1597,1567,1402,1332,1216$, 1119, 1052. UV/Vis $\left(\lambda_{\max }, \mathrm{nm}, \varepsilon, \mathrm{cm}^{-1} \mathrm{M}^{-1}\right): 259(25,000), 362(23,000) .{ }^{1} \mathrm{H}$ NMR $\left(300 \mathrm{MHz}, d_{6}\right.$-DMSO): $\delta_{H} 3.81$, 3.84 and $3.99(18 \mathrm{H}, 3 \mathrm{~s}, \mathrm{OMe}$ and $\mathrm{NMe}), 6.32(2 \mathrm{H}, \mathrm{s}, \mathrm{H} 6), 6.53(2 \mathrm{H}, \mathrm{s}, \mathrm{H} 2), 6.72(1 \mathrm{H}, \mathrm{br} \mathrm{s}, \mathrm{CH}), 6.96(2 \mathrm{H}, \mathrm{m}, \mathrm{aryl}$ $\mathrm{H}), 7.15\left(3 \mathrm{H}, \mathrm{m}\right.$, aryl H), $10.26(2 \mathrm{H}, \mathrm{s}, \mathrm{CHO}) .{ }^{13} \mathrm{C} \mathrm{NMR}\left(75 \mathrm{MHz}, d_{6}\right.$-DMSO): $\delta_{\mathrm{c}} 36.3$ (2C, NMe), 43.0 (CH), 56.0 and 57.0 (4C, OMe); 90.8 (2C, aryl CH), 111.6, 118.9 and 122.4 (aryl C), 125.6 (aryl CH), 126.9 (aryl C), 127.8 and 128.8 (4C, phenyl CH), 133.7 (2C, aryl CH), 145.2, 152.7 and 159.3 (aryl C), 187.8 (2C, CHO). MS (+El, $m / z$, \%): 527 (M, 11), 526 (31), 498 (13), 497 (29), 493 (21), 292 (88), 278 (96), 249 (47), 219 (54), 105 (63). HRMS $(\mathrm{m} / \mathrm{z})$ : Calcd for $\mathrm{C}_{31} \mathrm{H}_{30} \mathrm{~N}_{2} \mathrm{NaO}_{6}[\mathrm{M}+\mathrm{Na}]^{+}$, 549.1996. Found: 549.1985.

1,4-Bis[di(5',7'-dimethoxy-1'-methylindol-3'-yl)methyl]benzene (28). 5,7-Dimethoxy-1-methylindole 1 (0.20 $\mathrm{g}, 1.05 \mathrm{mmol})$, terephthalaldehyde $(50 \mathrm{mg}, 0.37 \mathrm{mmol})$ and glacial acetic acid $(4 \mathrm{~mL})$ were combined and stirred at room temperature for $5.5 \mathrm{~h}$. The resulting precipitate was filtered, washed with water and dried affording 1,4-bis[di(5',7'-dimethoxy-1'-methylindol-3'-yl)methyl]benzene $\mathbf{2 8}(0.16 \mathrm{~g}, 71 \%)$ as an off-white solid. Recrystallisation from acetone and petroleum ether yielded colourless crystals, $\mathrm{mp} 271-273^{\circ} \mathrm{C} . \operatorname{IR}\left(\mathrm{v}_{\max }, \mathrm{cm}^{-1}\right)$ : $1584,1497,1455,1412,1281,1207,1148,1117,1050$. UV/Vis $\left(\lambda_{\max }, \mathrm{nm}, \varepsilon, \mathrm{cm}^{-1} \mathrm{M}^{-1}\right): 265(31,000), 302$ (22,000), 306 (21,000). ${ }^{1} \mathrm{H}$ NMR (300 MHz, CDCl 3$): \delta_{H} 3.61,3.86$ and $3.87(36 \mathrm{H}, \mathrm{s}$, OMe and NMe), $5.60(2 \mathrm{H}, \mathrm{br}$ s, CH), 6.27 and $6.33(8 \mathrm{H}, \mathrm{d}, J 2.1 \mathrm{~Hz}, \mathrm{H} 6$ and $\mathrm{H} 4), 6.35(4 \mathrm{H}, \mathrm{s}, \mathrm{H} 2), 7.23\left(4 \mathrm{H}, \mathrm{s}\right.$, aryl H). ${ }^{13} \mathrm{C} \mathrm{NMR}(75 \mathrm{MHz}$, $\left.\mathrm{CDCl}_{3}\right): \delta_{\mathrm{C}} 36.1$ (4C, NMe), $39.9(2 \mathrm{C}, \mathrm{CH}), 55.3$ and 55.6 (8C, OMe), 93.2 and $94.1(8 \mathrm{C}$, aryl $\mathrm{CH}), 118.1$ and 122.4 (8C, aryl C), 128.5 (4C, phenyl CH), 129.1 (4C, aryl C), 129.6 (4C, aryl CH), 141.9 (2C, phenyl C), 147.9 and 153.9 (8C, aryl C). MS (+EI, m/z, \%): 863 (M, 0.01), 217 (16), 161 (37), 121 (90). HRMS (m/z): Calcd for $\mathrm{C}_{52} \mathrm{H}_{54} \mathrm{~N}_{4} \mathrm{NaO}_{8}$ $[\mathrm{M}+\mathrm{Na}]^{+}, 885.3834$. Found: 885.3790 .

Dimethyl 4,4'-[[4-chlorophenyl]methylene]bis[5,7-dimethoxyindole]-2,2'-dicarboxylate (29). A solution of methyl 5,7-dimethoxyindole-2-carboxylate $5(0.15 \mathrm{~g}, 0.64 \mathrm{mmol})$ and p-chlorobenzaldehyde $(90 \mathrm{mg}, 0.64$ $\mathrm{mmol})$ in anhydrous methanol $(10.0 \mathrm{~mL})$ was stirred at room temperature with concentrated $\mathrm{HCl}(3 \mathrm{drops})$ for $5 \mathrm{~h}$. The resulting precipitate was filtered through a frit, washed with water, and dried to give the title compound $(0.16 \mathrm{~g}, 84 \%)$ as a white powder, $\mathrm{mp} \mathrm{265-268}{ }^{\circ} \mathrm{C}$. IR $\left(v_{\max }, \mathrm{cm}^{-1}\right): 3441,3278,1721,1702,1590$, 1550, 1440, 1319, 1244, 1222, 1155, 986, 749. UV/Vis $\left(\lambda_{\max }, \mathrm{nm}, \varepsilon, \mathrm{cm}^{-1} \mathrm{M}^{-1}\right): 242(53,700), 278(20,200), 297$ (34,900), 336 (9,600). ${ }^{1} \mathrm{H}$ NMR $\left(300 \mathrm{MHz}_{\mathrm{C}} \mathrm{CDCl}_{3}\right): \delta_{H} 3.48,3.83$ and 3.94 (each $\left.6 \mathrm{H}, \mathrm{s}, \mathrm{OMe}\right), 6.51(2 \mathrm{H}, \mathrm{s}, \mathrm{H} 6)$, $6.56(1 \mathrm{H}, \mathrm{s}$, bridging $\mathrm{CH}), 6.68(2 \mathrm{H}, \mathrm{d}, J 2.3 \mathrm{~Hz}, \mathrm{H3}), 7.07$ and 7.18 (each $2 \mathrm{H}, \mathrm{d}, J 8.3 \mathrm{~Hz}, p$-chlorophenyl), 8.84 $\left(2 \mathrm{H}\right.$, bs, NH). ${ }^{13} \mathrm{C} \mathrm{NMR}\left(75 \mathrm{MHz}, \mathrm{CDCl}_{3}\right): \delta_{\mathrm{C}} 41.6$ (bridging $\mathrm{CH}$ ), 51.7, 55.4 and 58.1 (OMe), 95.8 (C6), 109.2 (C3), 127.8 and 130.2 (p-chlorophenyl CH), 116.9, 124.4, 126.6, 128.6, 131.1, 142.3, 145.4 and 152.2 (aryl C), 162.1 $\left(\mathrm{CO}_{2} \mathrm{Me}\right) . \mathrm{MS}(+\mathrm{El}, \mathrm{m} / \mathrm{z}, \%): 595\left(\mathrm{M}+1,{ }^{37} \mathrm{Cl}, 11\right), 594\left(\mathrm{M},{ }^{37} \mathrm{Cl}, 37\right), 593\left(\mathrm{M}+1,{ }^{35} \mathrm{Cl}, 33\right), 592\left(\mathrm{M},{ }^{35} \mathrm{Cl}, 100\right), 563$ (24), 562 (20), 561 (61), 327 (26), 326 (30), 325 (60). Anal. calcd for $\mathrm{C}_{31} \mathrm{H}_{29} \mathrm{ClN}_{2} \mathrm{O}_{8}$ : C, 62.8; H, 4.9; N, 4.7. Found: C, $62.9 ; \mathrm{H}, 4.7 ; \mathrm{N}, 4.8 \%$.

Dimethyl 4,4'-[[4-methoxyphenyl]methylene]bis[5,7-dimethoxyindole]-2,2'-dicarboxylate (30). A mixture of methyl 5,7-dimethoxyindole-2-carboxylate 5 (0.34 g, $1.45 \mathrm{mmol}$ ) and p-methoxybenzaldehyde (99 mg, 0.73 
$\mathrm{mmol})$ in anhydrous methanol $(5.0 \mathrm{~mL})$ was stirred at room temperature with concentrated $\mathrm{HCl}(1 \mathrm{drop})$ for 2 $\mathrm{h}$. The resulting precipitate was filtered through a frit, washed with a little methanol, then water, and dried to give the title compound $(0.39 \mathrm{~g}, 88 \%)$ as a white powder, m.p. $256-259{ }^{\circ} \mathrm{C}$ (methanol). IR $\left(\mathrm{v}_{\max }, \mathrm{cm}^{-1}\right): 3443$, $1710,1588,1538,1509,1438,1319,1246,1206,1088,984$. UV/Vis $\left(\lambda_{\max }, \mathrm{nm}, \varepsilon, \mathrm{cm}^{-1} \mathrm{M}^{-1}\right): 242(55,500), 298$ $(35,100), 338$ (9,930). ${ }^{1} \mathrm{H}$ NMR $\left(300 \mathrm{MHz}_{\mathrm{CDCl}}\right): \delta_{\mathrm{H}} 3.44(6 \mathrm{H}, \mathrm{s}, \mathrm{OMe}), 3.78(3 \mathrm{H}, \mathrm{s}, \mathrm{OMe}), 3.81$ and 3.94 (each $6 \mathrm{H}, \mathrm{s}, \mathrm{OMe}), 6.51(2 \mathrm{H}, \mathrm{s}, \mathrm{H} 6), 6.55(1 \mathrm{H}, \mathrm{s}$, bridging $\mathrm{CH}), 6.61(2 \mathrm{H}, \mathrm{d}, J 2.3 \mathrm{~Hz}, \mathrm{H} 3), 6.78$ and 7.06 (each $2 \mathrm{H}, \mathrm{d}, J$ $8.3 \mathrm{~Hz}, p$-methoxyphenyl), $8.81(2 \mathrm{H}, \mathrm{bs}, \mathrm{NH}) .{ }^{13} \mathrm{C} \mathrm{NMR}\left(75 \mathrm{MHz}, \mathrm{CDCl}_{3}\right): \delta_{\mathrm{C}} 41.9$ (bridging $\left.\mathrm{CH}\right), 52.1(\mathrm{OMe}), 55.8$ (p-methoxyphenyl OMe), 55.9 and 58.9 (OMe), 96.7 (C6), 110.0 (C3), 113.9 and 130.4 ( $p$-methoxyphenyl CH), 118.8, 125.0, 126.9, 129.2, 136.2, 145.6, 152.7 and 158.2 (aryl C), 162.7 ( CO $_{2} \mathrm{Me}$ ). MS (MALDI, $\left.m / z, \%\right): 588$ (M, 54), 587 (100). Anal. calcd for $\mathrm{C}_{32} \mathrm{H}_{32} \mathrm{~N}_{2} \mathrm{O}_{9}: \mathrm{C}, 65.3 ; \mathrm{H}, 5.5 ; \mathrm{N}, 4.8$. Found: $\mathrm{C}, 65.3 ; \mathrm{H}, 5.6 ; \mathrm{N}, 4.8 \%$.

Dimethyl 3,4'-[[4-chlorophenyl]methylene]bis[5,7-dimethoxyindole]-2,2'-dicarboxylate (31). A solution of methyl 5,7-dimethoxyindole-2-carboxylate $1(0.15 \mathrm{~g}, 0.64 \mathrm{mmol})$ and p-chlorobenzaldehyde $(46 \mathrm{mg}, 0.33$ $\mathrm{mmol})$ in anhydrous chloroform $(15 \mathrm{~mL})$ was heated at reflux with phosphoryl chloride $(0.1 \mathrm{~mL}, 1 \mathrm{mmol})$ for 2 h. The resulting solution was washed sequentially with iced water and dilute $\mathrm{NaOH}$, and dried $\left(\mathrm{MgSO}_{4}\right)$. The solvent was then evaporated in vacuo and the remaining residue was purified via gravity column chromatography $\left(\mathrm{CH}_{2} \mathrm{Cl}_{2}\right)$ to give the title compound $(70 \mathrm{mg}, 37 \%)$ as a white powder, $\mathrm{mp} 221-224{ }^{\circ} \mathrm{C}$. IR $\left(v_{\max }\right.$, $\left.\mathrm{cm}^{-1}\right): 3418,3347,1698,1584,1536,1440,1436,1323,1251,1201,1155$. UV/Vis $\left(\lambda_{\max }, \mathrm{nm}, \varepsilon, \mathrm{cm}^{-1} \mathrm{M}^{-1}\right): 241$ (48,000), 273 (18,000), 298 (34,000), 339 (10,000). ${ }^{1} \mathrm{H}$ NMR $\left(300 \mathrm{MHz}, \mathrm{CDCl}_{3}\right): \delta_{\mathrm{H}} 3.31,3.45,3.79,3.82,3.89$ and 3.95 (each $3 \mathrm{H}, \mathrm{s}, \mathrm{OMe}), 6.29$ and 5.83 (each $1 \mathrm{H}, \mathrm{d}, J 1.9 \mathrm{~Hz} \mathrm{H6}$ and $\mathrm{H} 4$ respectively), 6.52 (1H, s, H6'), 6.56 $\left(1 \mathrm{H}, \mathrm{d}, J 2.3 \mathrm{~Hz}, \mathrm{H} 3^{\prime}\right), 6.99(1 \mathrm{H}, \mathrm{s}$, bridging $\mathrm{CH}), 7.16(4 \mathrm{H}, \mathrm{m}, p$-chlorophenyl), 8.88 and 8.90 (each $1 \mathrm{H}, \mathrm{bs}, \mathrm{NH}$ and $\mathrm{NH}^{\prime}$ ). ${ }^{13} \mathrm{C} \mathrm{NMR}\left(75 \mathrm{MHz}, \mathrm{CDCl}_{3}\right): \delta_{\mathrm{C}} 40.9$ (bridging $\mathrm{CH}$ ), 52.0, 52.2, 55.4, 55.8, 55.9 and 58.6 (OMe), 95.2, 96.4 and 97.5 (C4, C6, and C6'), 109.2 (C3'), 128.5 and 130.9 (p-chlorophenyl $\mathrm{CH}$ ), 162.5 and 162.45 (들 $\left.{ }_{2} \mathrm{Me}\right)$, 117.1, 123.4, 123.9, 124.9, 125.4, 127.3, 128.6, 128.9, 132.0, 142.8, 146.0, 147.0, 153.1 and 154.9 (aryl C). MS (+El, m/z, \%): $595\left({ }^{37} \mathrm{Cl}, 9\right), 594\left(\mathrm{M},{ }^{37} \mathrm{Cl}, 29\right), 593(28), 592\left(\mathrm{M},{ }^{35} \mathrm{Cl}, 67\right), 591$ (12), 561 (14), 560 (9), 536 (12), 535 (40), 534 (37), 533 (100), 532 (20), 531 (17), 530 (17). Anal. calcd for $\mathrm{C}_{31} \mathrm{H}_{29} \mathrm{ClN}_{2} \mathrm{O}_{8}$ : C, 62.8; H, 4.9; N, 4.7. Found: C, 62.8; $\mathrm{H}, 4.9 ; \mathrm{N}, 4.9 \%$.

Dimethyl 4,4'-[[4-chlorophenyl]methylene]bis[5,7-dimethoxy- $N$-methylindole]-2,2'-dicarboxylate (32). A mixture of methyl 5,7-dimethoxy- $N$-methyl-indole-2-carboxylate $6(0.26 \mathrm{~g}, 1.05 \mathrm{mmol})$ and $p$-chlorobenzaldehyde $(148 \mathrm{mg}, 1.05 \mathrm{mmol})$ in anhydrous methanol $(3 \mathrm{~mL})$ was stirred at room temperature with concentrated $\mathrm{HCl}$ (1 drop) for $1.5 \mathrm{~h}$. The resulting precipitate was filtered through a frit, washed with water, and dried to give the title compound $(0.304 \mathrm{~g}, 93 \%)$ as a white powder, $\mathrm{mp} 178-183^{\circ} \mathrm{C}$. IR $\left(\mathrm{v}_{\max }, \mathrm{cm}^{-1}\right): 1711$, $1583,1458,1325,1240,1204,1175,1089,991$. UV/Vis $\left(\lambda_{\max }, \mathrm{nm}, \varepsilon, \mathrm{cm}^{-1} \mathrm{M}^{-1}\right): 244(57,700), 280(21,600), 300$ (33,800), 347 (11,200). ${ }^{1} \mathrm{H}$ NMR $\left(300 \mathrm{MHz}_{\mathrm{CDCl}}\right): \delta_{\mathrm{H}} 3.48,3.78$ and 3.91 (each $\left.6 \mathrm{H}, \mathrm{s}, \mathrm{OMe}\right), 4.28(6 \mathrm{H}, \mathrm{s}, \mathrm{NMe})$, $6.48(2 \mathrm{H}, \mathrm{s}, \mathrm{H} 6), 6.53(1 \mathrm{H}, \mathrm{s}$, bridging $\mathrm{CH}), 6.88(2 \mathrm{H}, \mathrm{s}, \mathrm{H} 3), 7.05$ and 7.17 (each $2 \mathrm{H}, \mathrm{d}, J 8.3 \mathrm{~Hz}, p$-chlorophenyl). ${ }^{13} \mathrm{C} \mathrm{NMR}\left(75 \mathrm{MHz}, \mathrm{CDCl}_{3}\right.$ ): $\delta_{\mathrm{C}} 34.9$ (NMe), 41.9 (bridging $\mathrm{CH}$ ), 51.8, 56.0 and 58.4 (OMe), 97.0 (C6), 111.5 (C3), 128.2 and 130.6 (p-chlorophenyl CH), 116.8, 127.0, 128.4, 128.6, 131.4, 143.0, 147.8 and 152.1 (aryl C), 163.0 $\left(\underline{C O}_{2} \mathrm{Me}\right) . \mathrm{MS}(+\mathrm{El}, \mathrm{m} / z, \%): 621$ (M, 4), 464 (13), 453 (14), 440 (68), 426 (100), 371 (22), 332 (13), 321 (18), 266 (29), 252 (42), 228 (79). Anal. calcd for $\mathrm{C}_{33} \mathrm{H}_{33} \mathrm{ClN}_{2} \mathrm{O}_{8}$ : C, 63.8; H, 5.4; N, 4.5. Found: C, 63.6; H, 5.2; N, 4.6\%.

Dimethyl 4,4'-[(4-chlorophenyl)methylene]bis[1-tert-butyloxycarbonyl-5,7-dimethoxyindole]-2,2'-dicarboxylate (33). A mixture of dimethyl p-chlorophenyl-4,4'-di(5,7-dimethoxyindolyl)methane-2,2'-dicarboxylate 29 $(0.76 \mathrm{mg}, 0.13 \mathrm{mmol})$, di-(t-butyl)carbonate $(121 \mathrm{mg}, 0.55 \mathrm{mmol})$ in anhydrous acetonitrile $(5 \mathrm{~mL})$ was stirred at room temperature with a catalytic quantity of $\mathrm{N}, \mathrm{N}$-dimethylaminopyridine for $2.5 \mathrm{~h}$. The solvent was then evaporated in vacuo and the remaining yellow syrup purified via suction column chromatography $\left(\mathrm{CH}_{2} \mathrm{Cl}_{2}\right)$ to give the title compound ( $65 \mathrm{mg}, 64 \%$ ) as a white powder, $\mathrm{mp}>133{ }^{\circ} \mathrm{C}$ (decomp.). IR $\left(v_{\max }, \mathrm{cm}^{-1}\right): 1770,1721$, 
$1589,1546,1488,1437,1394,1371,1336,1257,1231 \mathrm{v}, 1157,1081$. UV/Vis $\left(\lambda_{\max }, \mathrm{nm}, \varepsilon, \mathrm{cm}^{-1} \mathrm{M}^{-1}\right): 243$ (50,900), 296 (31,200), 343 (11,100). ${ }^{1} \mathrm{H}$ NMR $\left(300 \mathrm{MHz}, \mathrm{CDCl}_{3}\right): \delta_{\mathrm{H}} 1.64\left(18 \mathrm{H}, \mathrm{s}, \mathrm{CMe}_{3}, 3.50,3.81\right.$, and 3.90 (each $6 \mathrm{H}, \mathrm{s}, \mathrm{OMe}), 6.51(1 \mathrm{H}, \mathrm{s}$, bridging $\mathrm{CH}), 6.54(2 \mathrm{H}, \mathrm{s}, \mathrm{H} 6), 6.78(2 \mathrm{H}, \mathrm{s}, \mathrm{H} 3), 7.02(2 \mathrm{H}, \mathrm{d}, J 8.3 \mathrm{~Hz}, p$ chlorophenyl), $7.19\left(2 \mathrm{H}, \mathrm{d}, J 8.7 \mathrm{~Hz}, p\right.$-chlorophenyl). ${ }^{13} \mathrm{C} \mathrm{NMR}\left(75 \mathrm{MHz}, \mathrm{CDCl}_{3}\right)$ : $\delta_{\mathrm{c}} 41.9$ (bridging $\left.\mathrm{CH}\right), 27.8$ $\left(\mathrm{CMe}_{3}\right), 52.3,56.0$ and 58.0 (OMe), $84.9\left(\mathrm{CMe}_{3}\right), 97.1$ (C6), 112.0 (C3), 128.4 and 130.6 (p-chlorophenyl $\mathrm{CH}$ ), 116.7, 124.3, 127.7, 128.7, 131.7, 142.2, 146.4 and 153.3 (aryl C), 151.4 (CO), 161.6 (CO $\left.{ }_{2} \mathrm{Me}\right)$. MS (MALDI, m/z, \%): 790 (26), 691 (M-Boc, 14), 592 (M-2 x Boc, 100). Anal. calcd for $\mathrm{C}_{41} \mathrm{H}_{45} \mathrm{ClN}_{2} \mathrm{O}_{12}: \mathrm{C}, 62.1 ; \mathrm{H}, 5.7 ; \mathrm{N}, 3.5$. Found: $C, 61.8 ; \mathrm{H}, 5.9 ; \mathrm{N}, 3.56 \%$.

(Indol-3-yl)-bis(5',7'-dimethoxy-1'-methylindol-3'-yl)methane (35). 5,7-Dimethoxy-1-methylindole 1 (0.20 g, $1.05 \mathrm{mmol})$, indole-3-carbaldehyde $34(0.11 \mathrm{~g}, 0.79 \mathrm{mmol})$ and glacial acetic acid $(4 \mathrm{~mL})$ were combined and stirred at room temperature for $4 \mathrm{~h}$ under an inert gas atmosphere. The resulting precipitate was filtered, washed with water and dried, yielding (indol-3-yl)-di(5',7'-dimethoxy-1'-methylindol-3'-yl)methane 35 (67 mg, $25 \%)$ as an off-white solid. Recrystallisation from dichloromethane and petroleum ether afforded a white solid which turned pink on exposure to air, $\mathrm{mp} 224-225^{\circ} \mathrm{C} . \mathrm{IR}\left(v_{\max } \mathrm{cm}^{-1}\right): 3411,3343,1584,1495,1456,1413$, 1281, 1204, 1147, 1117, 1044. UV/Vis $\left(\lambda_{\max }, \mathrm{nm}, \varepsilon, \mathrm{cm}^{-1} \mathrm{M}^{-1}\right): 274(21,000), 293(19,000), 303(17,000) .{ }^{1} \mathrm{H}$ NMR $\left(300 \mathrm{MHz}_{\mathrm{CDCl}}\right): \delta_{\mathrm{H}} 3.68,3.85$ and $3.88(18 \mathrm{H}, 3 \times \mathrm{s}, \mathrm{OMe}$ and $\mathrm{NMe}), 5.92(1 \mathrm{H}, \mathrm{br} \mathrm{s}, \mathrm{CH}), 6.29(2 \mathrm{H}, \mathrm{d}, J$ $\left.2.0 \mathrm{~Hz}, \mathrm{H} 6^{\prime}\right), 6.46\left(2 \mathrm{H}, \mathrm{s}, \mathrm{H} 2^{\prime}\right), 6.48\left(2 \mathrm{H}, \mathrm{d}, J 2.0 \mathrm{~Hz}, \mathrm{H} 4^{\prime}\right), 6.76(1 \mathrm{H}, \mathrm{d}, J 2.0 \mathrm{~Hz}, \mathrm{H} 2), 6.99(1 \mathrm{H}, \mathrm{m}, \mathrm{H} 5), 7.16(1 \mathrm{H}, \mathrm{m}$, H6), $7.35(1 \mathrm{H}, \mathrm{d}, J 8.2 \mathrm{~Hz}, \mathrm{H} 4), 7.47(1 \mathrm{H}, \mathrm{d}, J 7.7 \mathrm{~Hz}, \mathrm{H} 7), 7.87(1 \mathrm{H}, \mathrm{br}, \mathrm{NH}) .{ }^{13} \mathrm{C} \mathrm{NMR}\left(75 \mathrm{MHz}, \mathrm{CDCl}_{3}\right): \delta_{\mathrm{C}} 30.9$ (CH), 36.1 (2C, NMe), 55.3 and 55.8 (4C, OMe), 93.3 and 94.0 (4C, aryl CH), 110.8 (aryl CH), $117.6(2 \mathrm{C}$, aryl C), 118.8 (aryl CH), 119.7 (aryl C), 120.2 and 121.5 (aryl CH), 122.4 (2C, aryl C), 123.3 (aryl CH), 127.2 (aryl C), 129.1 (2C, aryl C), 129.4 (2C, aryl CH), 136.7 (aryl C), 147.9 and 153.7 (4C, aryl C). MS (+El, m/z, \%): 510 (M, 19), 509 (100), 494 (46), 391 (17), 317 (38), 191 (90), 176 (73). HRMS (m/z): Calcd for $\mathrm{C}_{31} \mathrm{H}_{31} \mathrm{~N}_{3} \mathrm{NaO}_{4}[\mathrm{M}+\mathrm{Na}]^{+}$, 532.2207. Found: 532.2206.

(5,7-Dimethoxyindol-4-yl)-bis(5',7'-dimethoxy-1'-methylindol-3'-yl)methane (36). 5,7-Dimethoxy-1methylindole 1 (0.20 g, $1.05 \mathrm{mmol})$, 5,7-dimethoxyindole-4-carbaldehyde 10 (0.16 g, $0.79 \mathrm{mmol})$ and glacial acetic acid $(4 \mathrm{~mL})$ were combined and stirred at room temperature overnight. The resulting precipitate was filtered, washed with water and dried, yielding an off-white powder $(0.19 \mathrm{~g}) .{ }^{1} \mathrm{H}$ NMR spectroscopy showed that the powder contained (5,7-dimethoxyindol-4-yl)-di(5',7'-dimethoxy-1'-methylindol-3'-yl)methane 36 in $53 \%$ yield and unreacted 5,7-dimethoxyindole-4-carbaldehyde 10 (18\% of the original amount). Radial chromatography using dichloromethane afforded (5,7-dimethoxyindol-4-yl)-di(5',7'-dimethoxy-1'-methylindol3'-yl)methane (36) $(0.11 \mathrm{~g}, 37 \%)$ as a purple glass. Recrystallisation from dichloromethane and petroleum ether gave purple crystals, $\mathrm{mp} 270-271{ }^{\circ} \mathrm{C} . \mathrm{IR}\left(\mathrm{v}_{\max }, \mathrm{cm}^{-1}\right): 3406,1583,1493,1457,1412,1314,1282,1205$, 1145, 1114, 1047. UV/Vis $\left(\lambda_{\max }, \mathrm{nm}, \varepsilon, \mathrm{cm}^{-1} \mathrm{M}^{-1}\right)$ : 269 (13,000), $299(12,000), 304(11,000) .{ }^{1} \mathrm{H} \mathrm{NMR}(300 \mathrm{MHz}$, $\left.\mathrm{CDCl}_{3}\right): \delta_{\mathrm{H}} 3.65(6 \mathrm{H}, \mathrm{s}, \mathrm{OMe}$ or NMe), $3.77(3 \mathrm{H}, \mathrm{s}, \mathrm{OMe}), 3.85(12 \mathrm{H}, \mathrm{s}, \mathrm{NMe}$ and/or OMe), 3.95 (3H, s, OMe), $6.23\left(2 \mathrm{H}, \mathrm{d}, J 2.1 \mathrm{~Hz}, \mathrm{H} 6^{\prime}\right), 6.34(1 \mathrm{H}, \mathrm{dd}, J 2.3,3.0 \mathrm{~Hz}, \mathrm{H3}), 6.37(1 \mathrm{H}, \mathrm{t}, J 1.1 \mathrm{~Hz}, \mathrm{CH}), 6.44\left(2 \mathrm{H}, \mathrm{d}, J 2.1 \mathrm{~Hz}, \mathrm{H} 4^{\prime}\right)$, $6.51(1 \mathrm{H}, \mathrm{s}, \mathrm{H} 6), 6.54\left(2 \mathrm{H}, \mathrm{d}, J 1.1 \mathrm{~Hz}, \mathrm{H} 2{ }^{\prime}\right), 6.90(1 \mathrm{H}, \mathrm{m}, \mathrm{H} 2), 8.07(1 \mathrm{H}, \mathrm{m}, \mathrm{NH}) .{ }^{13} \mathrm{C} \mathrm{NMR}\left(75 \mathrm{MHz}, \mathrm{CDCl}_{3}\right): \delta_{c}$ 31.2 (CH), 36.1 (2C, NMe), 55.3 (2C, OMe), 55.3 (OMe), 55.6 (2C, OMe), 59.5 (OMe), 93.3 (aryl CH), 93.5 and 93.8 (4C, aryl CH), 103.8 (aryl CH), 117.5 (2C, aryl C), 117.5 (aryl C), 122.2 (2C, aryl C), 123.0 (aryl C), 123.2 (aryl CH), 128.7 (aryl C), 129.5 (2C, aryl CH), 129.7 (2C, aryl C), 144.5 (aryl C), 147.7 (2C, aryl C), 150.7 (aryl C), 153.5 (2C, aryl C). MS (+El, m/z, \%): 570 (M, 15), 569 (51), 554 (34), 391 (63), 191 (80), 176 (94), 148 (74). HRMS $(\mathrm{m} / \mathrm{z})$ : Calcd for $\mathrm{C}_{33} \mathrm{H}_{35} \mathrm{~N}_{3} \mathrm{NaO}_{6}[\mathrm{M}+\mathrm{Na}]^{+}, 592.2418$. Found: 592.2453.

3,3',3"-Tris(5,7-dimethoxy-1-methylindolyl)methane (37). Method 1. 5,7-Dimethoxy-1-methylindole 1 (0.20 g, $1.05 \mathrm{mmol})$, 5,7-dimethoxy-1-methylindole-3-carbaldehyde 7 (0.17 g, $0.79 \mathrm{mmol}$ ) and glacial acetic acid (4 $\mathrm{mL}$ ) were combined and stirred at room temperature overnight. The resulting precipitate was filtered, 
washed with water and dried, yielding 3,3',3"-tri(5,7-dimethoxy-1-methylindolyl)methane 37 (0.14 g, $47 \%)$ as a pale pink solid. Recrystallisation from dichloromethane and petroleum ether afforded a white powder, $\mathrm{mp}$ $245-246{ }^{\circ} \mathrm{C}$. IR $\left(v_{\max }, \mathrm{cm}^{-1}\right): 1584,1488,1456,1412,1281,1205,1147,1116,1048,810$. UV/Vis $\left(\lambda_{\max }, \mathrm{nm}, \varepsilon, \mathrm{cm}\right.$ $\left.{ }^{1} \mathrm{M}^{-1}\right)$ : 266 (16,000), 273 (15,000), 300 (17,000), 307 (16,000). ${ }^{1} \mathrm{H}$ NMR $\left(300 \mathrm{MHz}, \mathrm{CDCl}_{3}\right): \delta_{\mathrm{H}} 3.68,3.86$ and 3.88 $(27 \mathrm{H}, 3 \mathrm{~s}, \mathrm{OMe}$ and $\mathrm{NMe}), 5.78(1 \mathrm{H}, \mathrm{br} \mathrm{s}, \mathrm{CH}), 6.28(3 \mathrm{H}, \mathrm{d}, J 2.0 \mathrm{~Hz}, \mathrm{H} 6), 6.46(3 \mathrm{H}, \mathrm{s}, \mathrm{H} 2), 6.46(3 \mathrm{H}, \mathrm{d}, J 2.0 \mathrm{~Hz}$, H4. ${ }^{13} \mathrm{C} \mathrm{NMR} \mathrm{(75} \mathrm{MHz,} \mathrm{CDCl} 3$ ): $\delta_{\mathrm{C}} 30.9(\mathrm{CH}), 36.1$ (3C, NMe), 55.3 and 55.8 (6C, OMe, 93.6 and 93.9 (6C, aryl $\mathrm{CH}), 117.8,122.5$ and 129.3 (9C, aryl C), 129.5 (3C, aryl CH), 147.9 and 153.7 (6C, aryl C). MS (+EI, m/z, \%): 584 (M, 27), 583 (79), 568 (68), 392 (55), 391 (100), 192 (80), 191 (84), 176 (71). Anal. calcd for $\mathrm{C}_{34} \mathrm{H}_{37} \mathrm{~N}_{3} \mathrm{O}_{6}: \mathrm{C}, 70.0$; $H, 6.4 ; N, 7.2$. Found: $C, 69.7 ; H, 6.5 ; N, 7.0$.

Method 2. 5,7-Dimethoxy-1-methylindole 1 (0.20 g, $1.05 \mathrm{mmol})$ was dissolved in AR grade methanol (7 mL). Triethyl orthoformate $(0.58 \mathrm{~mL}, 3.49 \mathrm{mmol})$ was added, followed by a trace amount of 4-toluenesulfonic acid, and the reaction mixture was heated at reflux for $30 \mathrm{~min}$. After cooling, the resulting precipitate was filtered, washed with $10 \%$ aqueous ammonia solution followed by water and dried, affording 3,3',3"-tri(5,7-dimethoxy1-methylindolyl)methane $37(0.18 \mathrm{~g}, 88 \%)$ as a white solid.

3,3',3"-Tris(5,7-dimethoxyindolyl)methane (38). 5,7-Dimethoxyindole 2 (0.20 g, $1.13 \mathrm{mmol})$ was dissolved in AR grade methanol $(5 \mathrm{~mL})$. Triethyl orthoformate $(1.24 \mathrm{~mL}$, excess) was added, followed by a trace amount of 4-toluenesulfonic acid, and the reaction mixture was heated at reflux for $1 \mathrm{~h}$. After cooling, the resulting precipitate was filtered, washed with $10 \%$ aqueous ammonia solution followed by water and dried, affording 3,3',3"-tri(5,7-dimethoxyindolyl)methane $38(75 \mathrm{mg}, 37 \%)$ as a pale orange solid, $\mathrm{mp} 205-206{ }^{\circ} \mathrm{C}$. IR $\left(\mathrm{V}_{\max }, \mathrm{cm}^{-}\right.$ $\left.{ }^{1}\right): 3323,1571,1494,1455,1317,1201,1145,1130,1049,937,814$. UV/Vis $\left(\lambda_{\max }, \mathrm{nm}, \varepsilon, \mathrm{cm}^{-1} \mathrm{M}^{-1}\right): 267$ (17,000), 293 (14,000), 302 (12,000). ${ }^{1} \mathrm{H}$ NMR (300 MHz, CDCl 3$): \delta_{H} 3.68$ and $3.91(18 \mathrm{H}, 2 \mathrm{~s}, \mathrm{OMe}), 5.91(1 \mathrm{H}, \mathrm{m}$, $\mathrm{CH}), 6.32(3 \mathrm{H}, \mathrm{d}, J 2.1 \mathrm{~Hz}, \mathrm{H} 6), 6.50(1 \mathrm{H}, \mathrm{m}, \mathrm{H} 4), 6.71(3 \mathrm{H}, \mathrm{m}, \mathrm{H} 2), 7.97(3 \mathrm{H}, \mathrm{br} \mathrm{m}, \mathrm{NH}) .{ }^{13} \mathrm{C} \mathrm{NMR}(75 \mathrm{MHz}$, $\left.\mathrm{CDCl}_{3}\right): \delta_{\mathrm{C}} 31.5(\mathrm{CH}), 55.3$ and $55.9(6 \mathrm{C}, \mathrm{OMe}), 93.6$ and $93.8(6 \mathrm{C}$, aryl $\mathrm{CH}), 119.6$ and $122.5(6 \mathrm{C}$, aryl C), 123.3 (3C, aryl CH), 127.8, 146.3 and 154.3 (9C, aryl C). MS (+EI, m/z, \%): 542 (M, 2), 363 (11), 177 (45), 162 (49), 134 (100), 119 (95). HRMS (m/z): Calcd for $\mathrm{C}_{31} \mathrm{H}_{31} \mathrm{~N}_{3} \mathrm{NaO}_{6}[\mathrm{M}+\mathrm{Na}]^{+}, 564.2105$. Found: 564.2074.

3,3',3"-Tris(1-benzyl-5,7-dimethoxyindolyl)methane (39). 1-Benzyl-5,7-dimethoxyindole 3 (0.20 g, 0.75 mmol) was dissolved in AR grade methanol $(17 \mathrm{~mL})$. Triethyl orthoformate $(0.88 \mathrm{~mL}$, excess) was added, followed by a trace amount of 4-toluenesulfonic acid, and the reaction mixture was heated at reflux for $1.5 \mathrm{~h}$. After cooling, the resulting precipitate was filtered, washed with $10 \%$ aqueous ammonia solution followed by water and dried, affording 3,3',3"-tri(1-benzyl-5,7-dimethoxy-indolyl)methane $39(0.16 \mathrm{~g}, 77 \%)$ as a white solid. Recrystallisation from dichloromethane and petroleum ether yielded a white powder, $m p 262-263{ }^{\circ} \mathrm{C}$. IR $\left(v_{\max }\right.$, $\left.\mathrm{cm}^{-1}\right): 1615,1585,1493,1453,1426,1282,1204,1165,1149,1049,812,700$. UV/Vis $\left(\lambda_{\max }, \mathrm{nm} \varepsilon, \mathrm{cm}^{-1} \mathrm{M}^{-1}\right)$ : 266 (19,000), 274 (17,000), 301 (18,000), $307(18,000) .{ }^{1} \mathrm{H} N M R\left(300 \mathrm{MHz}, \mathrm{CDCl}_{3}\right): \delta_{\mathrm{H}} 3.56$ and $3.76(18 \mathrm{H}, 2 \mathrm{~s}$, OMe), $5.40\left(6 \mathrm{H}, \mathrm{s}, \mathrm{CH}_{2}\right), 5.86(1 \mathrm{H}, \mathrm{br} \mathrm{s}, \mathrm{CH}), 6.25(3 \mathrm{H}, \mathrm{d}, J 2.0 \mathrm{~Hz}, \mathrm{H} 6), 6.47(\mathrm{~d}, J 2.0 \mathrm{~Hz}, \mathrm{H} 4), 6.62(3 \mathrm{H}, \mathrm{s}, \mathrm{H} 2)$, $6.98\left(6 \mathrm{H}, \mathrm{m}\right.$, aryl H), $7.18\left(9 \mathrm{H}, \mathrm{m}\right.$, aryl H). ${ }^{13} \mathrm{C} \mathrm{NMR}\left(75 \mathrm{MHz} \mathrm{CDCl}_{3}\right): \delta_{\mathrm{C}} 31.5(\mathrm{CH}), 52.1\left(3 \mathrm{C}, \mathrm{CH}_{2}\right), 55.2$ and 55.6 (6C, OMe), 93.5 and 94.5 (6C, aryl CH), 118.2 and 122.0 (6C, aryl C), 126.3 (6C, aryl CH), 126.7 (3C, aryl CH), 128.2 (6C, aryl CH), 129.0 (3C, aryl CH), 129.5, 140.1, 147.7 and 153.9 (12C, aryl C). MS (+El, $m / z, \%): 812(\mathrm{M}$, 13), 811 (24), 796 (10). HRMS ( $m / z)$ : Calcd for $\mathrm{C}_{52} \mathrm{H}_{49} \mathrm{~N}_{3} \mathrm{NaO}_{6}[\mathrm{M}+\mathrm{Na}]^{+}, 834.3513$. Found: 834.3458.

Dimethyl 3,3'-[indol-3-ylmethylene]bis[5,7-dimethoxyindole]-2,2'-dicarboxylate (40). A solution of methyl 5,7-dimethoxyindole-2-carboxylate $2(0.10 \mathrm{~g}, 0.43 \mathrm{mmol}$ ) and indole-3-carbaldehyde 34 (62 $\mathrm{mg}, 0.43 \mathrm{mmol})$ in anhydrous methanol $(3 \mathrm{~mL})$ was stirred at room temperature with concentrated $\mathrm{HCl}(1 \mathrm{drop})$ for $24 \mathrm{~h}$. The resulting precipitate was filtered through a frit, washed with water, and dried to give the title compound (58 $\mathrm{mg}, 46 \%)$ as a white powder, $\mathrm{mp} 220-223^{\circ} \mathrm{C}$. IR $\left(v_{\max }, \mathrm{cm}^{-1}\right): 3454,3434,3379,3316,1702,1590,1543,1456$, 1438, 1323, 1312, 1245, 1197, 1156. UV/Vis $\left(\lambda_{\max }, \mathrm{nm}, \varepsilon, \mathrm{cm}^{-1} \mathrm{M}^{-1}\right): 239(49,400), 273(21,300), 293(31,600)$, 
$336(10,400) .{ }^{1} \mathrm{H}$ NMR (300 MHz, CDCl 3 ): $\delta_{H} 3.87,3.82$, and 3.11 (each $\left.6 \mathrm{H}, \mathrm{s}, \mathrm{OMe}\right), 5.94\left(2 \mathrm{H}, \mathrm{bs}, \mathrm{H} 4\right.$ and $\left.\mathrm{H} 4{ }^{\prime}\right)$, $6.23\left(2 \mathrm{H}, \mathrm{d}, J 1.9 \mathrm{~Hz}, \mathrm{H} 6\right.$ and H6'), $6.67(1 \mathrm{H}, \mathrm{d}, J 1.1 \mathrm{~Hz}, \mathrm{H} 2 "), 7.10$ and 6.90 (each $1 \mathrm{H}, \mathrm{dt}, J$ 1.1, $7.9 \mathrm{~Hz}, \mathrm{H} 5^{\prime \prime}$ and H6"), $7.33(2 \mathrm{H}, \mathrm{dt}, J 1.1,7.9 \mathrm{~Hz}, \mathrm{H} 4$ " and H7"), $7.49(1 \mathrm{H}, \mathrm{d}, J 1.5 \mathrm{~Hz}$, bridging $\mathrm{CH}), 7.90(1 \mathrm{H}, \mathrm{bs}, \mathrm{NH}$ "), $8.90(2 \mathrm{H}$, bs, $\mathrm{NH}$ and $\left.\mathrm{NH}^{\prime}\right)$. The signals at 8.90 and $7.90 \mathrm{ppm}$ exchanged with $\mathrm{D}_{2} \mathrm{O}$. ${ }^{1} \mathrm{H} \mathrm{NMR}\left(300 \mathrm{MHz}, d_{6}, \mathrm{DMSO}\right): \delta_{H}$ 3.81, 3.71, and 3.05 (each $6 \mathrm{H}, \mathrm{s}, \mathrm{OMe}), 5.85$ (2H, bs, H4 and H4'), $6.25\left(2 \mathrm{H}, \mathrm{s}, \mathrm{H} 6\right.$ and H6'), $6.54\left(1 \mathrm{H}, \mathrm{s}, \mathrm{H} 2^{\prime \prime}\right)$, 7.00 and 6.75 (each $1 \mathrm{H}, \mathrm{d}, J 8.3 \mathrm{~Hz}, \mathrm{H} 5$ " and H6"), 7.36 and 7.08 (each $1 \mathrm{H}, \mathrm{d}, J 8.3 \mathrm{~Hz}, \mathrm{H} 4$ " and H7"), 7.38 (1H, s, bridging $\mathrm{CH}), 10.71\left(1 \mathrm{H}, \mathrm{bs}, \mathrm{NH}^{\prime \prime}\right), 11.19\left(2 \mathrm{H}, \mathrm{bs}, \mathrm{NH}\right.$ and $\left.\mathrm{NH}^{\prime}\right)$. The signals at 11.19 and 10.71 ppm exchanged with $\mathrm{D}_{2} \mathrm{O} .{ }^{13} \mathrm{C}$ NMR (75 MHz, CDCl 3 ): $\delta_{\mathrm{C}} 31.5$ (bridging $\mathrm{CH}$ ), 51.5, 54.5 and 55.8 (OMe), 94.5 (C4 and C4'), 96.7 (C6 and $\mathrm{C6}^{\prime}$ ), 111.6, 118.6, 119.3, 121.3 and 123.6 (indolyl $\mathrm{CH}$ ), 117.9, 123.2, 124.0, 125.3, 127.9, 128.3, 137.0, 147.3 and 154.0 (aryl C), 162.0 ( CO $_{2} \mathrm{Me}$ ). MS (+El, m/z, \%): 598 (25), 597 (M, 71), 539 (33), 538 (100), 507 (16), 506 (49), 502 (21), 235 (45), 203 (48), 174 (27), 144 (23), 117 (21). Anal. calcd for $\mathrm{C}_{33} \mathrm{H}_{31} \mathrm{~N}_{3} \mathrm{O}_{8}: \mathrm{C}, 66.3 ; \mathrm{H}, 5.2 ; \mathrm{N}$, 7.0. Found: $C, 66.3 ; H, 5.1 ; N, 7.0 \%$.

Reaction of methyl 5,7-dimethoxyindole-2-carboxylate (5) with methyl 4-formyl-5,7-dimethoxyindole-2carboxylate (11). A mixture of methyl 5,7-dimethoxyindole-2-carboxylate 5 (0.180 g, $0.765 \mathrm{mmol})$ and methyl 4-formyl-5,7-dimethoxyindole-2-carboxylate 11 (0.106 g, $0.403 \mathrm{mmol}$ ) in anhydrous methanol (4 mL) was heated at reflux with concentrated $\mathrm{HCl}(2 \mathrm{drops})$ for $3.5 \mathrm{~h}$. The resulting precipitate was filtered through a frit, washed with a little methanol, then water, and dried to give an isomeric mixture $(0.227 \mathrm{~g}, 83 \%)$ of the 3,3',4",triindolylmethane 41 and the 3,3',3"-triindolylmethane 42 in a 88:12 ratio, as a white powder, mp $244-247{ }^{\circ} \mathrm{C}$. IR $\left(v_{\max }, \mathrm{cm}^{-1}\right): 3468,3335,1716,1589,1539,1451,1437,1329,1310,1247,1199,1156$. UV/Vis $\left(\lambda_{\max }, \mathrm{nm}, \varepsilon\right.$, $\mathrm{cm}^{-1} \mathrm{M}^{-1}$ ): 241 (66,800), 279 (27,600), 297 (46,400), 338 (14,400). MS (MALDI, m/z, \%): 715 (M, 47$), 714(100)$. Anal. calcd for $\mathrm{C}_{37} \mathrm{H}_{37} \mathrm{~N}_{3} \mathrm{O}_{12} .0 .5 \mathrm{H}_{2} \mathrm{O}: \mathrm{C}, 61.3 ; \mathrm{H}, 5.3 ; \mathrm{N}, 5.8$. Found: $\mathrm{C}, 61.1 ; \mathrm{H}, 5.0 ; \mathrm{N}, 5.7 \%$.

Purification of the isomeric mixture via preparative thin layer chromatography led to the isolation and characterisation of compound 41, and recovery of a trace amount of compound $\mathbf{4 2 .}$

Trimethyl 3'4"-methylidynetris[5,7-dimethoxyindole]-2,2',2"-tricarboxylate (41). ${ }^{1} \mathrm{H} \mathrm{NMR}\left(300 \mathrm{MHz} \mathrm{CDCl}_{3}\right): \delta$ H 3.02 (3H, s, OMe), 3.16 and 3.39 (each $3 \mathrm{H}, \mathrm{bs}, \mathrm{OMe}), 3.71$ (3H, s, OMe), 3.75 (6H, s, OMe), 3.85, 3.87, 3.92 (each $3 \mathrm{H}, \mathrm{s}, \mathrm{OMe}$ ), 5.78 and 5.95 (each $1 \mathrm{H}, \mathrm{bs}, \mathrm{H} 4$ and $\mathrm{H}^{\prime}$ ), 6.23 and 6.27 (each $1 \mathrm{H}, \mathrm{d}, \mathrm{J} 1.9 \mathrm{~Hz}, \mathrm{H} 3$ " and H6"), $6.52\left(2 \mathrm{H}, \mathrm{s}, \mathrm{H} 6\right.$ and $\left.\mathrm{H}^{\prime}\right), 7.73(1 \mathrm{H}, \mathrm{s}$, bridging $\mathrm{CH}), 8.87\left(2 \mathrm{H}, \mathrm{bs}, \mathrm{NH}\right.$ and $\left.\mathrm{NH}^{\prime}\right), 8.94(1 \mathrm{H}, \mathrm{bs}, \mathrm{NH}) .{ }^{13} \mathrm{C} \mathrm{NMR}(75$ $\mathrm{MHz}_{\mathrm{CDCl}}$ ): $\delta_{\mathrm{C}} 34.6$ (bridging $\mathrm{CH}$ ), 51.8, 51.9, 52.0, 52.1, 55.0, 55.2, 55.8, 55.9 and 59.1 (OMe), 94.9 and 95.3 (C4), 97.1, 97.3, and 97.4 (C6), 109.4 (C3), 118.8, 123.1, 123.3, 123.8, 124.1, 125.0, 126.2, 127.2, 127.3, 129.2, $129.5,145.7,146.9,147.0,153.1,154.7,155.1$ (aryl C), 162.4, 162.5 and $162.9\left(\mathrm{CO}_{2} \mathrm{Me}\right)$.

Trimethyl 3',3"-methylidynetris[5,7-dimethoxyindole]-2,2',2"-tricarboxylate (42). IR ( $\left.v_{\max }, \mathrm{cm}^{-1}\right): 3468,1714$, $1652,1634,1455,1248,1199,1158 ;{ }^{1} \mathrm{H}$ NMR $\left(300 \mathrm{MHz}_{2} \mathrm{CDCl}_{3}\right): \delta_{\mathrm{H}} 3.87,3.78$, and 2.98 (each $\left.6 \mathrm{H}, \mathrm{s}, \mathrm{OMe}\right), 5.95$ $(3 \mathrm{H}, \mathrm{d}, J 1.9 \mathrm{~Hz}, \mathrm{H} 4), 6.24(3 \mathrm{H}, \mathrm{d}, J 1.9 \mathrm{~Hz}, \mathrm{H} 6), 8.15(1 \mathrm{H}, \mathrm{s}$, bridging $\mathrm{CH}), 8.94(3 \mathrm{H}, \mathrm{bs}, \mathrm{NH}) .{ }^{13} \mathrm{C} \mathrm{NMR}(75 \mathrm{MHz}$, $\mathrm{CDCl}_{3}$ ): $\delta_{\mathrm{C}} 32.4$ (bridging $\mathrm{CH}$ ), 51.4, 54.5, and 55.4 (OMe), 94.2 (C4), 96.7 (C6), 122.5, 123.8, 125.3, 128.7, 146.6 and 154.7 (aryl C), $162.1\left(\mathrm{CO}_{2} \mathrm{Me}\right)$.

Reaction of methyl 5,7-dimethoxyindole-2-carboxylate (5) with triethyl orthoformate: formation of compounds 41 and 42. A mixture of methyl 5,7-dimethoxyindole-2-carboxylate (5) (0.250 g, $1.06 \mathrm{mmol})$ and triethyl orthoformate $(0.10 \mathrm{~mL}, 0.60 \mathrm{mmol})$ in anhydrous methanol $(3 \mathrm{~mL})$ was heated at reflux, with a catalytic quantity of $p$-toluenesulfonic acid, for $24 \mathrm{~h}$. The resulting precipitate was filtered through a frit, washed with a little methanol, then water, and dried to give a 98:2 isomeric mixture $(0.202 \mathrm{~g}, 80 \%)$ of the 3,3',4"-triindolylmethane $\mathbf{4 1}$ and 3,3',3"-triindolylmethane $\mathbf{4 2}$ as a white powder.

2,4-Dimethoxy-5-methyl-6-(5',7'-dimethoxy-1'-methylindol-3'-yl)benzo[1,2-b]carbazole (43). 5,7-Dimethoxy1-methylindole $1(0.20 \mathrm{~g}, 1.05 \mathrm{mmol})$, phthalaldehyde (70 $\mathrm{mg}, 0.52 \mathrm{mmol}$ ) and glacial acetic acid $(4 \mathrm{~mL})$ were combined and stirred at room temperature for $4.5 \mathrm{~h}$. The resulting precipitate was filtered, washed with 
water and dried, yielding 2,4-dimethoxy-5-methyl-6-(5',7'-dimethoxy-1'-methylindol-3'-yl)benzo[1,2b]carbazole 43 as a bright yellow powder $(87 \mathrm{mg}, 34 \%)$. Recrystallisation from ethyl acetate and petroleum ether afforded bright yellow crystals, $\mathrm{mp} 191-192{ }^{\circ} \mathrm{C}$. IR $\left(v_{\max }, \mathrm{cm}^{-1}\right): 1589,1498,1457,1302,1278,1207,1151$, 1116, 1057. UV/Vis $\left(\lambda_{\max }, \mathrm{nm}, \varepsilon, \mathrm{cm}^{-1} \mathrm{M}^{-1}\right): 278$ (47,000), 287 (59,000), 307 (14,000), 322 (7,500), $337(4,000)$, 407 (5,600), 419 (5,600). ${ }^{1} \mathrm{H}$ NMR (300 MHz, CDCl $): \delta_{H} 3.53$ (3H, br s, 5-NMe), 3.58 (3H, s, 5'-OMe), $3.90(3 \mathrm{H}$, s, 4-OMe), $3.98\left(6 \mathrm{H}, \mathrm{s}, 2-\mathrm{OMe}\right.$ and 7'-OMe), $4.16\left(3 \mathrm{H}, \mathrm{s}, 1^{\prime}-\mathrm{NMe}\right), 6.20\left(1 \mathrm{H}, \mathrm{d}, J 2.1 \mathrm{~Hz}, \mathrm{H} \mathbf{H}^{\prime}\right), 6.38(1 \mathrm{H}, \mathrm{d}, J 2.1$ Hz, H6'), $6.66(1 \mathrm{H}, \mathrm{d}, J 2.1 \mathrm{~Hz}, \mathrm{H} 3), 7.02\left(1 \mathrm{H}, \mathrm{s}, \mathrm{H} 2^{\prime}\right), 7.30(1 \mathrm{H}, \mathrm{m}, \mathrm{H} 8), 7.35(1 \mathrm{H}, \mathrm{d}, J 2.1 \mathrm{~Hz}, \mathrm{H} 1), 7.36(1 \mathrm{H}, \mathrm{m}$, $\mathrm{H} 9), 7.82(1 \mathrm{H}, \mathrm{d}, J 8.4 \mathrm{~Hz}, \mathrm{H} 7), 8.05(1 \mathrm{H}, \mathrm{dd}, J 1.4,8.2 \mathrm{~Hz}, \mathrm{H} 10), 8.54(1 \mathrm{H}, \mathrm{s}, \mathrm{H} 11) .{ }^{13} \mathrm{C} \mathrm{NMR}\left(75 \mathrm{MHz} \mathrm{CDCl}_{3}\right): \delta_{\mathrm{C}}$ 34.6 and 36.5 (NMe), 55.4, 55.7, 55.8 and 56.0 (OMe), 93.3, 94.9, 95.0 and 100.0 (aryl CH), 111.1 and 112.0 (aryl C), 117.7 (aryl CH), 121.8 (aryl C), 122.4 (aryl CH), 124.5 (aryl C), 124.6 and 125.8 (aryl CH), 126.4 and 128.0 (aryl C), 128.2 (aryl CH), 129.4 (aryl C), 130.8 (aryl CH), 132.1, 133.7, 141.9, 147.5, 148.1, 154.4 and 154.9 (aryl C). MS (+El, m/z, \%): 481 (M, 28), 480 (85), 465 (15). Anal. calcd for $\mathrm{C}_{30} \mathrm{H}_{28} \mathrm{~N}_{2} \mathrm{O}_{4}: \mathrm{C}, 75.0 ; \mathrm{H}, 5.9 ; \mathrm{N}, 5.8$. Found: $\mathrm{C}, 74.8 ; \mathrm{H}, 5.9 ; \mathrm{N}, 5.6 \%$.

Dimethyl 2,6,8,12-tetrahydro-3,5,9,11-tetramethoxy-6,12-o-benzeno-diindolo[4,4a,3-bc:4',4a',3'-fg]cyclooctene-1,7-dicarboxylate (44). Method A. A stirred solution of methyl 5,7-dimethoxyindole-2-carboxylate 5 $(1.75 \mathrm{~g}, 7.46 \mathrm{mmol})$ and phthaldialdehyde $(0.50 \mathrm{~g}, 3.73 \mathrm{mmol})$ in anhydrous methanol $(15 \mathrm{~mL})$ was heated at reflux with concentrated $\mathrm{HCl}$ ( 2 drops) for $3 \mathrm{~h}$. After cooling to room temperature the resulting precipitate was filtered through a frit, washed with a little methanol, then water, and dried to give the title compound $(1.92 \mathrm{~g}, 90 \%)$ as a white powder, $\mathrm{mp} 316-319{ }^{\circ} \mathrm{C} . \mathrm{IR}\left(\mathrm{v}_{\max }, \mathrm{cm}^{-1}\right): 3468,3334,1687,1590,1538,1456,1436$, 1392, 1354, 1298, 1254, 1204, 1111, 1000. UV/Vis $\left(\lambda_{\max }, \mathrm{nm}, \varepsilon, \mathrm{cm}^{-1} \mathrm{M}^{-1}\right): 245(49,000), 279(18,000), 301$ (21,000), 345 (11,000). ${ }^{1} \mathrm{H}$ NMR $\left(300 \mathrm{MHz}, \mathrm{CDCl}_{3}\right): \delta_{H} 3.85,3.94$ and 4.08 (each $\left.6 \mathrm{H}, \mathrm{s}, \mathrm{OMe}\right), 6.46(2 \mathrm{H}, \mathrm{s}, \mathrm{H} 6)$, $6.90(2 \mathrm{H}, \mathrm{s}$, bridging $\mathrm{CH}), 7.12(2 \mathrm{H}, \mathrm{m}$, aryl $\mathrm{CH}), 7.48(2 \mathrm{H}, \mathrm{m}, \operatorname{aryl~} \mathrm{CH}), 8.66(2 \mathrm{H}, \mathrm{bs}, \mathrm{NH}) .{ }^{13} \mathrm{C} \mathrm{NMR}(75 \mathrm{MHz}$, $\mathrm{CDCl}_{3}$ ): $\delta_{\mathrm{C}} 40.0$ (bridging $\mathrm{CH}$ ), 52.1, 56.0 and 60.0 (OMe), 97.3 (C6), 127.5 and 130.3 (aryl CH), 117.9, 121.6, 124.2, 125.9, 127.1, 142.5, 145.6 and 150.0 (aryl C), 163.2 ( CO $\left._{2} M e\right)$. MS (MALDI, $\left.m / z, \%\right): 568$ (M, 100). Anal. calcd for $\mathrm{C}_{32} \mathrm{H}_{28} \mathrm{~N}_{2} \mathrm{O}_{8}: \mathrm{C}, 67.6 ; \mathrm{H}, 5.0 ; \mathrm{N}, 4.9$. Found: $\mathrm{C}, 67.9 ; \mathrm{H}, 5.0 ; \mathrm{N}, 4.9 \%$.

Method B. A stirred solution of methyl 5,7-dimethoxyindole-2-carboxylate 5 (0.11 g, $0.48 \mathrm{mmol})$ and phthaldialdehyde (32 mg, $0.24 \mathrm{mmol}$ ) in chloroform $(4 \mathrm{~mL})$ was treated with phosphoryl chloride $(0.02 \mathrm{~mL}, 0.2$ $\mathrm{mmol})$. The darkened solution was stirred at room temperature for 3 days before it was basified with $2 \mathrm{M}$ $\mathrm{NaOH}$ and extracted with dichloromethane. The organic extract was washed with water, dried $\left(\mathrm{MgSO}_{4}\right)$, and the solvent evaporated in vacuo to give the title compound $(0.13 \mathrm{~g}, 93 \%)$ as a white powder.

Method C. A mixture of methyl 5,7-dimethoxyindole-2-carboxylate 5 (0.19 $\mathrm{g}, \quad 0.79$ mmol) and phthaldialdehyde $(53 \mathrm{mg}, 0.24 \mathrm{mmol})$ in anhydrous methanol $(7 \mathrm{~mL})$, was stirred with $p$-toluenesulfonic acid $(10 \mathrm{mg})$, under nitrogen, for $4 \mathrm{~d}$. The resulting precipitate was filtered through a frit, washed with a little methanol, then water, and dried to give the title compound (150 mg, 100\%) as a white powder.

Dimethyl 2,6,8,12-tetrahydro-3,5,9,11-tetramethoxy-2,8-dimethyl-6,12-o-benzenodiindolo[4,4a,3-b,c:4',4a',3'-f,g]cyclooctene-1,7-dicarboxylate (46). A stirred solution of methyl 5,7-dimethoxy- $N$-methylindole-2carboxylate (6) $(0.230 \mathrm{~g}, 0.923 \mathrm{mmol})$ and phthaldialdehyde $(80 \mathrm{mg}, 0.60 \mathrm{mmol})$ in anhydrous methanol $(5 \mathrm{~mL})$ was stirred at ambient temperature with concentrated $\mathrm{HCl}(1$ drop) for $4 \mathrm{~h}$. The resulting precipitate was filtered through a frit, washed with water, and dried to give the title compound $(0.12 \mathrm{~g}, 44 \%)$ as a white powder, $\mathrm{mp} 356-358{ }^{\circ} \mathrm{C}$. IR $\left(v_{\max }, \mathrm{cm}^{-1}\right)$ : $1713,1586,1457,1394,1320,1288,1227,1202,1116$. UV/Vis $\left(\lambda_{\max }\right.$ $\left.\mathrm{nm}, \varepsilon, \mathrm{cm}^{-1} \mathrm{M}^{-1}\right): 247$ (46,500), 277 (15,500), 300 (17,000), $356(11,500) .{ }^{1} \mathrm{H} \mathrm{NMR}\left(300 \mathrm{MHz}, \mathrm{CDCl}_{3}\right): \delta_{\mathrm{H}} 3.80$, 3.89 and 4.05 (each $6 \mathrm{H}, \mathrm{bs}, \mathrm{OMe}), 4.14(6 \mathrm{H}, \mathrm{s}, \mathrm{NMe}), 6.40(2 \mathrm{H}, \mathrm{s}, \mathrm{H} 6), 6.73(2 \mathrm{H}, \mathrm{bs}$, bridging $\mathrm{CH}), 7.13(2 \mathrm{H}, \mathrm{dd}, J$ 3.4, $5.6 \mathrm{~Hz}$, aryl H), $7.45\left(2 \mathrm{H}\right.$, dd, J 3.4, $5.7 \mathrm{~Hz}$, aryl H). ${ }^{13} \mathrm{C} \mathrm{NMR}\left(75 \mathrm{MHz}, \mathrm{CDCl}_{3}\right): \delta_{\mathrm{C}} 34.7$ (NMe), 39.7 (bridging $\mathrm{CH}$ ), 52.0, 56.2, and 59.2 (OMe), 97.0 (C6), 127.5 and 130.0 (aryl $\mathrm{CH}$ ), 163.8 ( $\left.\mathrm{CO}_{2} \mathrm{Me}\right), 117.4,125.0,125.2$, 
126.2, 126.6, 142.4, 147.1 and 149.3 (aryl C). MS (+EI, m/z, \%): 596 (M, 25), 581 (26), 553 (23), 538 (31), 537 (100), 536 (19), 522 (21), 521 (39), 507 (32), 506 (26), 505 (17), 493 (18), 491 (24), 477 (22), 475 (22), 463 (32), 462 (21), 461 (22), 448 (29), 447 (49). Anal. calcd for $\mathrm{C}_{34} \mathrm{H}_{32} \mathrm{~N}_{2} \mathrm{O}_{8} . \mathrm{H}_{2} \mathrm{O}: \mathrm{C}, 66.4 ; \mathrm{H}, 5.6 ; \mathrm{N}, 4.6$. Found: $\mathrm{C}, 66.1 ; \mathrm{H}$, $5.3 ; \mathrm{N}, 4.6 \%$.

2,2-Bis(5',7'-dimethoxy-1'-methylindol-3'-yl)propane (47). 5,7-Dimethoxy-1-methylindole 1 (0.10 g, 0.53 $\mathrm{mmol})$, AR grade acetone ( $1 \mathrm{~mL}$, excess) and acetic anhydride $(4 \mathrm{~mL})$ were combined and heated at reflux in an inert gas atmosphere for $6 \mathrm{~h}$. No precipitate was formed so the reaction mixture was diluted with water and allowed to stand overnight. The resulting precipitate was filtered, washed with water and dried, affording a white solid. Radial chromatography using 50:50 dichloromethane: petroleum ether yielded 2,2-bis(5',7'dimethoxy-1'-methylindol-3'-yl)propane $47(20 \mathrm{mg}, 18 \%)$ as pale tan crystals, $\mathrm{mp} 168-169{ }^{\circ} \mathrm{C}$. IR $\left(\mathrm{V}_{\max }, \mathrm{cm}^{-1}\right)$ : 1580, 1495, 1456, 1413, 1305, 1258, 1212, 1149, 1116, 1045. UV/Vis $\left(\lambda_{\max }, \mathrm{nm}, \varepsilon, \mathrm{cm}^{-1} \mathrm{M}^{-1}\right): 221(42,000), 229$ (43,000), 266 (10,000), $272(10,000), 300$ (11,000), 307 (10,000). ${ }^{1} \mathrm{H}$ NMR $\left(300 \mathrm{MHz}, \mathrm{CDCl}_{3}\right): \delta_{\mathrm{H}} 1.83(6 \mathrm{H}, \mathrm{s}$, $\mathrm{Me}), 3.65,3.85$ and $3.93(18 \mathrm{H}, 3 \mathrm{~s}, \mathrm{OMe}$ and $\mathrm{NMe}), 6.22(2 \mathrm{H}, \mathrm{d}, J 2.3 \mathrm{~Hz}, \mathrm{H6}), 6.44(2 \mathrm{H}, \mathrm{d}, J 2.3 \mathrm{~Hz}, \mathrm{H} 4), 6.71(2 \mathrm{~h}$, s, H2). ${ }^{13} \mathrm{C} N M R\left(75 \mathrm{MHz}, d_{6}\right.$-DMSO): $\delta_{\mathrm{C}} 29.8$ (2C, Me), 34.1 (C), 36.1 (2C, NMe), 55.3 and 55.8 (4C, OMe), 93.5 and 95.0 (4C, aryl CH), 122.6 and 122.9 (4C, aryl C), 127.2 (2C, aryl CH), 128.4, 147.8 and 152.9 (6C, aryl C). MS (+El, $m / z, \%): 423$ (M, 4), 422 (14), 407 (39), 216 (13). HRMS (m/z): Calcd for $\mathrm{C}_{25} \mathrm{H}_{30} \mathrm{~N}_{2} \mathrm{NaO}_{4}[\mathrm{M}+\mathrm{Na}]^{+}, 445.2098$. Found: 445.2091.

6,8-Dimethoxy-1,3,3,5-tetramethyl-1,3-dihydrobenz[c, $d$ ]indole (48). 5,7-Dimethoxy-1-methylindole 1 (0.20 g, $1.05 \mathrm{mmol})$, AR grade acetone $(2 \mathrm{~mL}$, excess) and glacial acetic acid $(4 \mathrm{~mL})$ were combined and refluxed under an inert gas atmosphere for $7 \mathrm{~h}$. No precipitate was formed so the reaction mixture was diluted with water and allowed to stand overnight. The resulting colloidal solution was extracted with dichloromethane, and the organic phase was dried $\left(\mathrm{Na}_{2} \mathrm{SO}_{4}\right)$ and the solvent removed under reduced pressure yielding a brown oil. Radial chromatography using 50:50 dichloromethane: petroleum ether afforded 6,8-dimethoxy-1,3,3,5tetramethyl-1,3-dihydrobenz $[c d]$ indole $48(28 \mathrm{mg}, 10 \%)$ as a cream solid, $\mathrm{mp} 123-125^{\circ} \mathrm{C}$. IR $\left(\mathrm{V}_{\max }, \mathrm{cm}^{-1}\right): 1605$, $1513,1464,1409,1283,1234,1214,1116,1089,1053,1023$. UV/Vis $\left(\lambda_{\max } \mathrm{nm}, \varepsilon, \mathrm{cm}^{-1} \mathrm{M}^{-1}\right): 234(19,000), 249$ (22,000), 300 (4,800), $314(6,800), 328(9,300), 342(9,500), 355(4,700) .{ }^{1} \mathrm{H}$ NMR $\left(300 \mathrm{MHz}, \mathrm{CDCl}_{3}\right): \delta_{\mathrm{H}} 1.28$ (6H, s, Me), 2.28 (3H, d, J $1.1 \mathrm{~Hz}, \mathrm{Me}), 3.83$ (3H, s, 6-OMe), 3.90 (3H, s, 8-OMe), 3.94 (3H, s, NMe), 5.24 (1H, br d, J $1.1 \mathrm{~Hz}, \mathrm{H} 4), 6.26(1 \mathrm{H}, \mathrm{s}, \mathrm{H} 7), 6.68(1 \mathrm{H}, \mathrm{s}, \mathrm{H} 2) .{ }^{13} \mathrm{C} \mathrm{NMR}\left(75 \mathrm{MHz}, \mathrm{CDCl}_{3}\right): \delta_{\mathrm{c}} 22.5(5-\mathrm{Me}), 33.6(2 \mathrm{C}, 3-\mathrm{Me})$, 33.9 (C3), 35.5 (NMe), 55.5 (8-OMe), 59.4 (6-OMe), 94.8 (C7), 109.5 (C5a), 119.8 (C8a), 121.9 (C2a), 123.8 $\left(\mathrm{CH}_{2}\right), 127.3$ (C5), $\left.127.3 \mathrm{C} 8 \mathrm{~b}\right), 134.6$ (C4), 147.3 (C8), 149.4 (C6). MS (+EI, m/z, \%): 271 (M, 22 ), 256 (100), 241 (20). HRMS $(m / z)$ : Calcd for $\mathrm{C}_{17} \mathrm{H}_{21} \mathrm{NNaO}_{2}[\mathrm{M}+\mathrm{Na}]^{+}$, 294.1464. Found: 294.1457. Anal. calcd for $\mathrm{C}_{17} \mathrm{H}_{21} \mathrm{NO}_{2}: \mathrm{C}_{\text {, }}$ 75.2; $\mathrm{H}, 7.8 ; \mathrm{N}, 5.2$. Found: $\mathrm{C}, 74.7 ; \mathrm{H}, 8.0 ; \mathrm{N}, 4.7 \%$.

Methyl 1,5-dihydro-6,8-dimethoxy-3,5,5-trimethylbenz[cd]indole-2-carboxylate (49). A mixture of methyl 5,7-dimethoxyindole-2-carboxylate $5(0.15 \mathrm{~g}, 0.64 \mathrm{mmol})$ in 2,2-dimethoxypropane $(2 \mathrm{~mL})$ was heated at reflux with a catalytic quantity of $p$-toluenesulfonic acid for $16 \mathrm{~h}$. Water was then added and the resulting gum decanted from the solute, dissolved in ethyl acetate, washed sequentially with water and brine, dried $\left(\mathrm{MgSO}_{4}\right)$, and then the solvent evaporated in vacuo. The remaining red residue was purified via gravity column chromatography $\left(2 \% \mathrm{MeOH} / \mathrm{CH}_{2} \mathrm{Cl}_{2}\right)$ to give the title compound $(55 \mathrm{mg}, 34 \%)$ as a yellow powder, $\mathrm{mp}$ (softens at $\left.174{ }^{\circ} \mathrm{C}\right) 183-187{ }^{\circ} \mathrm{C} . \mathrm{IR}\left(\mathrm{v}_{\max }, \mathrm{cm}^{-1}\right): 3349,1686,1536,1456,1391,1336,1272,1253,1207,1172$, 997. UV/Vis $\left(\lambda_{\max }, \mathrm{nm}, \varepsilon, \mathrm{cm}^{-1} \mathrm{M}^{-1}\right): 245(15,000), 273$ (11,000), $301(13,000), 312(12,000), 366(6,100) .{ }^{1} \mathrm{H} N M R$ $\left(300 \mathrm{MHz}, \mathrm{CDCl}_{3}\right): \delta_{\mathrm{H}} 1.46(6 \mathrm{H}, \mathrm{s}, \mathrm{Me}), 2.30(3 \mathrm{H}, \mathrm{d}, J 1.1 \mathrm{~Hz}, \mathrm{Me}), 3.86,3.89$, and 3.94 (each 3H, s, OMe), 5.45 $(1 \mathrm{H}, \mathrm{d}, J 1.5 \mathrm{~Hz}, \mathrm{H} 4), 6.54(1 \mathrm{H}, \mathrm{s}, \mathrm{H} 7), 8.46(1 \mathrm{H}, \mathrm{bs}, \mathrm{NH}) .{ }^{13} \mathrm{C} \mathrm{NMR}\left(75 \mathrm{MHz}, \mathrm{CDCl}_{3}\right): \delta_{\mathrm{C}} 22.3$ and 28.5 (Me), 38.0 (C5), 51.4, 55.5, and 57.6 (OMe), 97.0 (C7), 141.0 (C4), 118.6, 120.1, 120.3, 120.5, 123.7, 127.7, 143.9 and 151.5 (aryl C), 161.9 ( $\left.\mathrm{CO}_{2} \mathrm{Me}\right) . \mathrm{MS}$ (+El, m/z, \%): 315 (M, 16), 300 (67), 269 (17), 268 (100), 182 (20), 134 (15), 
112 (15). HRMS $(\mathrm{m} / z)$ : Calcd for $\mathrm{C}_{18} \mathrm{H}_{21} \mathrm{NO}_{4}[\mathrm{M}]^{+}$, 315.1471. Found: 315.1473. Anal. calcd for $\mathrm{C}_{18} \mathrm{H}_{21} \mathrm{NO}_{4} .0 .25 \mathrm{H}_{2} \mathrm{O}: \mathrm{C}, 66.7 ; \mathrm{H}, 6.8 ; \mathrm{N}, 4.3$. Found: $\mathrm{C}, 66.9 ; \mathrm{H}, 6.7 ; \mathrm{N}, 4.0 \%$.

Methyl 4-(2'-hydroxy-2'-indane-1',3'-dionyl)-5,7-dimethoxyindole-2-carboxylate (50). A stirred suspension of methyl 5,7-dimethoxyindole-2-carboxylate $5(0.25 \mathrm{~g}, 1.1 \mathrm{mmol})$ and ninhydrin $(0.19 \mathrm{~g}, 1.1 \mathrm{mmol})$ in anhydrous methanol $(4 \mathrm{~mL}$ ) was heated at reflux with concentrated $\mathrm{HCl}$ (3 drops) for $1.5 \mathrm{~h}$. After cooling to room temperature, the resulting precipitate was filtered through a frit, washed with water, and dried to give the title compound $(0.38 \mathrm{~g}, 91 \%)$ as a yellow powder, $\mathrm{mp} 238-241^{\circ} \mathrm{C}$. IR $\left(v_{\max }, \mathrm{cm}^{-1}\right): 3412,3323,1744,1698,1584$, 1536, 1438, 1326, 1246, 1216, 1145, 973. UV/Vis $\left(\lambda_{\max }, \mathrm{nm}, \varepsilon, \mathrm{cm}^{-1} \mathrm{M}^{-1}\right): 241(42,500), 282(14,300), 297$ (17,300), 329 (5,600). ${ }^{1} \mathrm{H}$ NMR $\left(300 \mathrm{MHz}_{\mathrm{CDCl}}\right): \delta_{\mathrm{H}} 3.45,3.90$, and 3.95 (each $\left.3 \mathrm{H}, \mathrm{s}, \mathrm{OMe}\right), 6.28(1 \mathrm{H}, \mathrm{s}, \mathrm{H} 6)$, $7.86\left(2 \mathrm{H}, \mathrm{m}\right.$, ninhydrinyl), $7.90(1 \mathrm{H}, \mathrm{d}, J 2.3 \mathrm{~Hz}, \mathrm{H3}), 8.03\left(2 \mathrm{H}, \mathrm{m}\right.$, ninhydrinyl), $9.01(1 \mathrm{H}, \mathrm{bs}, \mathrm{NH}) .{ }^{1} \mathrm{H} \mathrm{NMR}(300$ $\mathrm{MHz}, d_{6}$-DMSO): $\delta_{H} 3.25,3.84$, and 3.87 (each $\left.3 \mathrm{H}, \mathrm{s}, \mathrm{OMe}\right), 6.52(1 \mathrm{H}, \mathrm{s}, \mathrm{H} 6), 6.89(1 \mathrm{H}, \mathrm{s}, \mathrm{OH}), 7.74(1 \mathrm{H}, \mathrm{s}, \mathrm{H} 3)$, $7.98\left(4 \mathrm{H}, \mathrm{s}\right.$, ninhydrinyl), $11.68(1 \mathrm{H}, \mathrm{bs}, \mathrm{NH})$. The signals at 6.89 and $11.68 \mathrm{ppm}$ exchanged with $\mathrm{D}_{2} \mathrm{O} .{ }^{13} \mathrm{C} \mathrm{NMR}$ (75 MHz, $d_{6}$-DMSO): $\delta_{c}$ 51.9, 56.1 and 57.5 (OMe), $78.3(\mathrm{COH}), 95.4$ (C6), 111.6 (C3), 123.6 and 136.5 (ninhydrinyl CH), 111.2, 125.9, 127.2, 127.7, 140.4, 147.7, 150.0 (aryl C), $161.9\left(\mathrm{CO}_{2} \mathrm{Me}\right), 199.3$ (CO). MS (+El, m/z, \%): 395 (M, 96), 363 (19), 320 (20), 304 (18), 265 (13), 262 (14), 235 (54), 230 (52), 204 (16), 203 (100), 144 (23), 105 (13), 104 (54). Anal. calcd for $\mathrm{C}_{21} \mathrm{H}_{17} \mathrm{NO}_{7}: \mathrm{C}, 63.8 ; \mathrm{H}, 4.3 ; \mathrm{N}, 3.5$. Found: $\mathrm{C}, 63.9 ; \mathrm{H}, 4.4 ; \mathrm{N}, 3.6$ \%.

Dimethyl 2,2-bis(5,7-dimethoxyindol-4-yl)indane-1,3-dione-2',2"-dicarboxylate (51). Methyl 5,7-dimethoxyindole-2-carboxylate $5(0.25 \mathrm{~g}, 1.1 \mathrm{mmol})$ and ninhydrin (95 $\mathrm{mg}, 0.53 \mathrm{mmol}$ ) in anhydrous toluene $(50 \mathrm{~mL}) \mathrm{was}$ heated at reflux with a catalytic quantity of $p$-toluenesulfonic acid for $2 \mathrm{~d}$ using a Dean-Stark apparatus. After cooling to room temperature, the resulting precipitate was filtered through a frit, washed with a little toluene, then light petroleum (the combined filtrate was kept aside, see below), and dried to give the title compound $(0.32 \mathrm{~g}, 98 \%)$ as a yellow powder, $\mathrm{mp} 238-240{ }^{\circ} \mathrm{C}$ (chloroform/light petroleum). IR $\left(v_{\max } \mathrm{cm}^{-1}\right): 3457,3343$, 1704, 1322, 1253, 1210, UV/Vis $\left(\lambda_{\max }, \mathrm{nm}, \varepsilon, \mathrm{cm}^{-1} \mathrm{M}^{-1}\right): 243(60,600), 277(19,300), 298(29,700), 334(8,850)$. ${ }^{1} \mathrm{H}$ NMR (300 MHz, CDCl 3 ): $\delta_{H}$ 3.51, 3.74 and 3.95 (each $\left.6 \mathrm{H}, \mathrm{s}, \mathrm{OMe}\right), 6.34\left(2 \mathrm{H}, \mathrm{d}, \mathrm{J} 2.3 \mathrm{~Hz}, \mathrm{H} 3{ }^{\prime}\right.$ and H3"), 6.44 $\left(2 \mathrm{H}, \mathrm{s}, \mathrm{H} 6^{\prime}\right.$ and $\left.\mathrm{H} 6^{\prime \prime}\right), 7.76$ and 7.98 (each $2 \mathrm{H}, \mathrm{m}$, ninhydrinyl), $8.75(2 \mathrm{H}, \mathrm{bs}, \mathrm{NH}) .{ }^{13} \mathrm{C} \mathrm{NMR}\left(75 \mathrm{MHz}^{\mathrm{C}} \mathrm{CDCl}_{3}\right): \delta_{\mathrm{C}}$ 52.0, 58.6, 56.0 (OMe), 63.2 (bridging C), 96.6 (C6' and C6"), 111.3 (C3' and C3"), 123.5 and 134.6 (ninhydrinyl $\mathrm{CH}), 113.4,125.7,127.0,129.1,142.3,147.4,152.1$ (aryl C), 162.4 ( CO$\left._{2} \mathrm{Me}\right), 197.7$ (CO). MS (+El, $\left.\mathrm{m} / z, \%\right): 612$ (M, 18), 577 (17), 551 (20), 369 (17), 368 (40), 367 (22), 353 (16), 339 (37), 313 (66), 299 (25), 285 (22), 265 (27), 264 (68), 262 (58), 239 (82), 238 (37), 236 (100). HRMS (m/z): Calcd for $\mathrm{C}_{33} \mathrm{H}_{28} \mathrm{~N}_{2} \mathrm{O}_{10}[\mathrm{M}]^{+}, 612.1744$. Found: 612.1748.

1-Pentanoyl-2,3-dihydroindole-2,3-dione (52). Valeryl chloride (10.30 g, $85.4 \mathrm{mmol})$ was added dropwise over $10 \mathrm{~min}$ to a stirred suspension of isatin $(12.0 \mathrm{~g}, 81.6 \mathrm{mmol})$ and pyridine $(8 \mathrm{~mL})$ in dichloromethane $(140 \mathrm{~mL})$ at room temperature. The mixture was heated at reflux for $1 \mathrm{~h}$ and then allowed to cool before the resulting red solution was washed briefly with dilute hydrochloric acid and dried $\left(\mathrm{MgSO}_{4}\right)$. The solvent was then reduced in vacuo to approximately $50 \mathrm{~mL}$ before light petroleum was added to effect the precipitation of the product, which was then filtered and washed with light petroleum. Recrystallization from cyclohexane gave the title compound $(15.8 \mathrm{~g}, 84 \%)$ as a bright yellow powder, $\mathrm{mp} 96-99^{\circ} \mathrm{C}$. IR $\left(\mathrm{v}_{\max }, \mathrm{cm}^{-1}\right): 1783,1757,1708,1609,1466$, 1331, 1169. UV/Vis $\left(\lambda_{\max }, \mathrm{nm}, \varepsilon, \mathrm{cm}^{-1} \mathrm{M}^{-1}\right): 239(18,600), 295(4,100) .{ }^{1} \mathrm{H} \mathrm{NMR}\left(300 \mathrm{MHz}, \mathrm{CDCl}_{3}\right): \delta_{\mathrm{H}} 0.97(3 \mathrm{H}, \mathrm{t}$, J $7.2 \mathrm{~Hz}, \mathrm{Me}$ ), 1.44 and 1.74 (each $\left.2 \mathrm{H}, \mathrm{m}, \mathrm{CH}_{2}\right), 3.10\left(2 \mathrm{H}, \mathrm{t}, J 7.5 \mathrm{~Hz}, \mathrm{CH}_{2}\right), 7.32$ and 7.71 (each $1 \mathrm{H}, \mathrm{t}, J 7.5 \mathrm{~Hz}$, phenyl), 7.78 and 8.43 (each $1 \mathrm{H}, \mathrm{d}, J 7.5 \mathrm{~Hz}$, phenyl). ${ }^{13} \mathrm{C} \mathrm{NMR}\left(75 \mathrm{MHz}, \mathrm{CDCl}_{3}\right.$ ): $\delta \mathrm{c} 13.7$ (Me), $22.1,26.1$ and $37.9\left(\mathrm{CH}_{2}\right), 118.2,125.2,125.9$ and 138.8 (aryl CH), 119.2 and 148.8 (aryl C), 157.7 and 173.0 (CONHCO), 180.2 (CO). MS (+El, m/z, \%): 231 (M, 4), 146 (100), 90 (28), 85 (44), 57 (52). Anal. calcd for $\mathrm{C}_{13} \mathrm{H}_{13} \mathrm{NO}_{3}: \mathrm{C}, 67.5 ; \mathrm{H}$, 5.7; N, 6.1. Found: C, 67.5; H, 5.8; N, $6.2 \%$. 
Dimethyl 5,5",7,7"-tetramethoxy-2'-oxo-[3,3':3'(2'H),3"-ter-1H-indole]-2,2"-dicarboxylate (53). Method A. A solution of methyl 5,7-dimethoxyindole-2-carboxylate 5 (0.25 g, $1.1 \mathrm{mmol})$ and $\mathrm{N}$-pentanoylisatin 52 (0.25 g, $1.1 \mathrm{mmol})$ in anhydrous methanol $(25 \mathrm{~mL})$ was heated at reflux with concentrated $\mathrm{HCl}(10 \mathrm{drops})$ for $7 \mathrm{~h}$. The resulting precipitate was filtered through a frit, washed with water, and dried to give the title compound (0.24 $\mathrm{g}, 75 \%)$ as a white powder, $\mathrm{mp} 299-30{ }^{\circ} \mathrm{C}$. IR $\left(v_{\max } \mathrm{cm}^{-1}\right): 3629,3449,3386,1715,1692,1587,1540,1316$, 1249, 1236, 1216. UV/Vis $\left(\lambda_{\max }, \mathrm{nm}, \varepsilon, \mathrm{cm}^{-1} \mathrm{M}^{-1}\right): 242(57,200), 298(37,400), 338(10,200) .{ }^{1} \mathrm{H} \mathrm{NMR}(300 \mathrm{MHz}$, $\left.\mathrm{CDCl}_{3}\right): \delta_{\mathrm{H}} 3.44,3.46,3.76$ and 3.77 (each $\left.3 \mathrm{H}, \mathrm{s}, \mathrm{OMe}\right), 3.94(6 \mathrm{H}, \mathrm{s}, \mathrm{OMe}), 5.79(1 \mathrm{H}, \mathrm{bs}, \mathrm{H} 3 "), 6.46$ (1H, s, H3), $6.50(2 \mathrm{H}, \mathrm{s}, \mathrm{H} 6$ and H6"), $6.81(1 \mathrm{H}, \mathrm{t}, J 7.5 \mathrm{~Hz}$, indolonyl), 6.87 and 7.12 (each $1 \mathrm{H}, \mathrm{d}, J 7.5 \mathrm{~Hz}$, indolonyl H4' and $\mathrm{H}^{\prime}$ ), $7.16\left(1 \mathrm{H}, \mathrm{t}, \mathrm{J} 7.5 \mathrm{~Hz}\right.$, indolonyl), $7.40\left(1 \mathrm{H}, \mathrm{bs}, \mathrm{NH}\right.$ ), 8.77 and 8.79 (each $1 \mathrm{H}, \mathrm{bs}, \mathrm{NH}$ and $\left.\mathrm{NH}^{\prime \prime}\right) .{ }^{1} \mathrm{H} N M R(300$ $\mathrm{MHz}, d_{6}$-DMSO): $\delta_{H} 3.24$ and 3.41 (each $3 \mathrm{H}, \mathrm{s}, \mathrm{OMe}$ ), 3.65 and 3.89 (each $6 \mathrm{H}, \mathrm{s}, \mathrm{OMe}$ ), 5.87 and 6.43 (each $1 \mathrm{H}$, s, $\mathrm{H3}$ and $\left.\mathrm{H} 3^{\prime \prime}\right), 6.56$ and 6.61 (each $1 \mathrm{H}, \mathrm{s}, \mathrm{H} 6$ and $\left.\mathrm{H} 6 "\right), 6.67(1 \mathrm{H}, \mathrm{t}, \mathrm{J} 7.5 \mathrm{~Hz}$, indolonyl), 6.77 and 6.92 (each $1 \mathrm{H}$, d, J $7.5 \mathrm{~Hz}$, indolonyl), $7.04(1 \mathrm{H}, \mathrm{t}, J 7.5 \mathrm{~Hz}$, indolonyl), $10.14(1 \mathrm{H}, \mathrm{bs}, \mathrm{NH}), 11.35$ and 11.48 (each $1 \mathrm{H}, \mathrm{bs}, \mathrm{NH}$ and $\left.\mathrm{NH}^{\prime \prime}\right) .{ }^{13} \mathrm{C}$ NMR (75 MHz, $d_{6}$-DMSO): $\delta_{c} 56.9$ (bridging $\mathrm{C}, \mathrm{C}^{\prime}$ ), 51.7, 51.8, 55.9, 56.0, 58.4 and 58.8 (OMe), 97.8 and 98.1 (C6 and C6"), 108.7 and 109.4 (C3 and C3"), 120.7, 124.6, 126.8 (indolonyl CH), 111.7, 116.4, 125.7, 125.8, 126.2, 127.1, 128.1, 136.6, 141.5, 146.2, 151.4 (aryl C), 161.6 and $161.7\left(\mathrm{CO}_{2} \mathrm{Me}\right), 178.6$ (CONH). MS (MALDI, $m / z, \%): 599$ (M, 48), 598 (100). Anal. calcd for $\mathrm{C}_{32} \mathrm{H}_{29} \mathrm{~N}_{3} \mathrm{O}_{9} . \mathrm{H}_{2} \mathrm{O}: \mathrm{C}, 62.2 ; \mathrm{H}, 5.1 ; \mathrm{N}, 6.8$. Found: C, $62.5 ; \mathrm{H}, 4.8 ; \mathrm{N}, 6.8 \%$.

Method B. A mixture of methyl 5,7-dimethoxyindole-2-carboxylate $2(0.16 \mathrm{~g}, 0.70 \mathrm{mmol})$ and methyl $N$-butyl2'-acetamidophenylglyoxylic ester $54(0.10 \mathrm{~g}, 0.39 \mathrm{mmol})$ in anhydrous methanol $(2 \mathrm{~mL})$ was heated at reflux with concentrated $\mathrm{HCl}$ ( 1 drop) for $3 \mathrm{~h}$. The resulting precipitate was filtered through a frit, washed with a little methanol, then water, and dried to give the title compound $(0.17 \mathrm{~g}, 79 \%)$ as a white powder.

Methyl 2'-(n-butylamido)phenylglyoxylate (54). A mixture of $N$-pentanoylisatin 52 (1.00 g, $4.32 \mathrm{mmol})$ in anhydrous methanol $(2 \mathrm{~mL}$ ) was heated at reflux with concentrated $\mathrm{HCl}(1$ drop) for $2 \mathrm{~h}$. The solvent was then evaporated and the remaining residue was purified via suction column chromatography (dichloromethane) to give the title compound $(0.64 \mathrm{~g}, 56 \%)$ as a bright yellow syrup. IR $\left(v_{\max }, \mathrm{cm}^{-1}\right): 3323,1742,1704,1655,1608$, 1584, 1530, 1450, 1204, 1161, 1000, 753. UV/Vis $\left(\lambda_{\max }, \mathrm{nm}, \varepsilon, \mathrm{cm}^{-1} \mathrm{M}^{-1}\right): 240(19,000), 266(8,000), 349(4,000)$. ${ }^{1} \mathrm{H}$ NMR $\left(300 \mathrm{MHz}, \mathrm{CDCl}_{3}\right): \delta_{\mathrm{H}} 0.95(3 \mathrm{H}, \mathrm{t}, J 7.2 \mathrm{~Hz}, \mathrm{Me}), 1.42$ and 1.74 (each $\left.2 \mathrm{H}, \mathrm{m}, \mathrm{CH}_{2}\right), 2.46(2 \mathrm{H}, \mathrm{t}, J 7.2 \mathrm{~Hz}$, $\left.\mathrm{CH}_{2}\right), 3.99(3 \mathrm{H}, \mathrm{s}, \mathrm{OMe}), 7.13(1 \mathrm{H}, \mathrm{dt}, J 1.1,7.5 \mathrm{~Hz}$, phenyl), $7.65(2 \mathrm{H}, \mathrm{m}$, phenyl), $8.82(1 \mathrm{H}, \mathrm{dd}, J 1.1,8.6 \mathrm{~Hz}$, phenyl), $11.07\left(1 \mathrm{H}\right.$, bs, NHCO). ${ }^{13} \mathrm{C} \mathrm{NMR}\left(75 \mathrm{MHz}_{\mathrm{CDCl}}\right): \delta_{\mathrm{c}} 13.6(\mathrm{Me}), 22.2,27.4$ and $38.3\left(\mathrm{CH}_{2}\right), 52.9$ $\left(\mathrm{CO}_{2} \mathrm{Me}\right), 120.8,122.5,133.5$ and 137.1 (aryl CH), 117.0 and 142.6 (aryl C), $163.8\left(\mathrm{CO}_{2} \mathrm{Me}\right), 173.0(\mathrm{CONH})$, 190.2 (CO). MS (+EI, m/z, \%): 263 (M, 2), 204 (64), 146 (24), 120 (100), 92 (31), 90 (22), 85 (23), 57 (63). HRMS $(\mathrm{m} / \mathrm{z})$ : Calcd for $\mathrm{C}_{14} \mathrm{H}_{17} \mathrm{NO}_{4}[\mathrm{M}]^{+}, 263.1158$. Found: 263.1159.

\section{Supplementary Material}

A checkcif file (compound $\mathbf{4 4}$ ) is available as Supplementary Data. Graphical NMR spectroscopic data are also presented for compounds 5, 15, 19, 44, 49, and 53 .

\section{Acknowledgements}

We thank the Australian Research Council for financial support. 


\section{References}

1. Black, D. StC.; Kumar, N.; Wong, L. C. H. Aust. J. Chem. 1986, 39, 15-20.

https://doi.org/10.1071/CH9860015

2. Black, D. StC. Synlett 1993, 246-252.

https://doi.org/10.1055/s-1993-22420

3. Black, D. StC.; Bowyer, M. C.; Kumar, N.; Mitchell, P. S. R. J.Chem.Soc., Chem. Commun. 1993, 819-821. https://doi.org/10.1039/c39930000819

4. Black, D. StC.; Bowyer, M. C.; Catalano, M. M.; Ivory, A. J.; Keller, P. A.; Kumar, N.; Nugent, S. J. Tetrahedron 1994, 50, 10497-10508.

https://doi.org/10.1016/S0040-4020(01)89590-9

5. Black, D. StC.; Bowyer, M. C.; Bowyer, P. K.; Ivory, A. J.; Kim, M.; Kumar, N.; McConnell, D. B.; Popiolek, M. Aust. J. Chem. 1994, 47, 1741-1750.

https://doi.org/10.1071/CH9941741

6. Black, D. StC.; Craig, D. C.; Kumar, N. Aust. J. Chem. 1996, 49, 311-318. https://doi.org/10.1071/CH9960311

7. Chen, R.; Bhadbhade, M.; Kumar, N.; Black, D. StC. Tetrahedron Lett. 2012, 53, 3337-3341. https://doi.org/10.1016/j.tetlet.2012.04.082

8. Somphol, K.; Chen, R.; Bhadbhade, M.; Kumar, N.; Black, D. StC. Synlett 2013, 24-28. https://doi.org/10.1055/s-0032-1317858

9. Chen, R.; Somphol, K.; Bhadbhade, M.; Kumar, N.; Black, D. StC. Synlett 2013, 1497-1500. http://doi.org/10.1055/s-0033-1338868

10. Condie, G. C.; Channon, M, F.; Ivory, A. J.; Kumar, N.; Black, D. StC. Tetrahedron 2005, 61, 4989-5004. https://doi.org/10.1016/i.tet.2005.03.048

11. Black, D. StC.; Craig, D. C.; Kumar, N. Tetrahedron Lett. 1991, 32, 1587-1590. https://doi.org/10.1016/S0040-4039(00)74279-1

12. Black, D. StC.; Channon, M. F.; Clayton, K. A.; Condie, G. C.; Harper, J. B.; Kumar, N.; Pchalek, K.; Wahyuningsih, T. D.; Arkivoc 2006, (vii), 67-75. https://doi.org/10.3998/ark.5550190.0007.707

13. Schöllkopf, U.; Lonsky, R.; Lehr, P. Liebigs Ann. Chem. 1985, 413-417. https://doi.org/10.1002/jlac.198519850217

14. Oliveira, D. d. J.; Coelho, F. Synth. Commun. 2000, 30, 2143-2159. https://doi.org/10.1080/00397910008087393

15. Dobrowolski, J.; Somphol, K.; Santoso, M.; Duong, H.; Gardner, C. R.; Kumar, N.; Black, D. StC. Aust. J. Chem. 2017, 70, 1188-1195.

https://doi.org/10.1071/CH17257

16. Joule, J. A. in Science of Synthesis; Thomas, E. J. Ed.; Georg Thieme Verlag: Stuttgart, 2000, Vol. 10, pp 361652.

http://doi.org/10.1055/sos-SD-110-00001

17. Trofimov, B. A.; Nedolya, N. A. in Comprehensive Heterocyclic Chemistry III; Jones, G. A.; Ramsden, C. A. Eds.; Elsevier: Oxford, 2008, Vol. 3, pp 45-267. https://doi.org/10.1016/B978-008044992-0.00302-3

18. Clezy, P. S.; Fookes, C. J. R.; Liepa, A. J. Aust. J. Chem. 1972, 25, 1979-1990. https://doi.org/10.1071/CH9721979 
19. Akgün, E.; Pindur, U.; Müller, J. J. Heterocycl. Chem. 1983, 20, 1303-1305.

https://doi.org/10.1002/jhet.5570200530

20. Schiffl, E.; Pindur, U. J. Heterocycl. Chem. 1986, 23, 651-656.

https://doi.org/10.1002/jhet.5570230303

21. Pindur, U.; Müller, J. J. Heterocycl. Chem. 1987, 24, 289-290.

https://doi.org/10.1002/jhet.5570240156

22. Witzel, H.; Pindur, U. J. Heterocycl. Chem. 1988, 25, 907-910.

https://doi.org/10.1002/jhet.5570250339

23. Gmeiner, P.; Kraxner, J.; Bollinger, B. Synthesis 1996, 1196-1198.

https://doi.org/10.1055/s-1996-4356

24. Kraxner, J.; Arlt, M.; Gmeiner, P. Synlett 2000, 125-127.

https://doi.org/10.1055/s-2000-6439

25. Rodrigues, A. M. S.; Rohée, C.; Fabre, T.; Batailler, N.; Sautel, F.; Carletti, I.; Nogues, S.; Suzuki, M. T.; Stien, D. Tetrahedron Lett. 2017, 58, 3172-3173.

https://doi.org/10.1016/i.tetlet.2017.07.005

26. Aghazadeh, M. Arkivoc 2019, (vi), 141-148.

https://doi.org/10.24820/ark.5550190.p011.012

27. Black, D. StC.; Craig, D. C.; Santoso, M. Tetrahedron Lett. 1999, 40, 6653-6656.

https://doi.org/10.1016/S0040-4039(99)01268-X

28. Zhao, L.; Li, Z.; Wirth, T. Chem. Lett. 2010, 39, 658-667.

https://doi.org/10.1246/cl.2010.658

29. Bergman, J.; Norrby, P. O.; Tilstam, U.; Venemalm, L. Tetrahedron 1989, 45, 5549-5564, and references cited therein.

https://doi.org/10.1016/S0040-4020(01)89501-6

30. Sakagami, M.; Muratake, H.; Natsume, M. Chem. Pharm. Bull. Japan 1994, 42, 1393-1398.

https://doi.org/10.1248/cpb.42.1393

31. Black, D. StC.; Moss, G. I.; Wong, L. C. H. Tetrahedron Lett. 1978, 2837-2838.

32. Cheah, W. C; Wood, K.; Black, D. StC.; Kumar, N. Tetrahedron 2011, 67, 7603-7610.

https://doi.org/10.1016/i.tet.2011.07.036

33. Hewawasam, P.; Meanwell, N. A. Tetrahedron Lett. 1994, 35, 7303-7306.

https://doi.org/10.1016/0040-4039(94)85299-5

34. Liu, J-X.; Zhu, Z-Q.; Yu, L.; Du, B-X.; Mei, G-J.; Shi, F. Synthesis 2018, 50, 3436-3444, and references cited therein.

https://doi.org/10.1055/s-0037-1609732

This paper is an open access article distributed under the terms of the Creative Commons Attribution (CC BY) license (http://creativecommons.org/licenses/by/4.0/)s 